Supporting Information for:

\title{
Enantioselective Hydrogenation of Diarylmethanimines for Synthesis of Chiral Diarylmethylamines
}

Duanyang Kong, Meina Li, Guofu Zi, Guohua Hou* and Yong He*

Key Laboratory of Radiopharmaceuticals, College of Chemistry, Beijing Normal University, Beijing 100875, China

\section{Contents:}

1. NMR spectra..................................................... 2 . 57

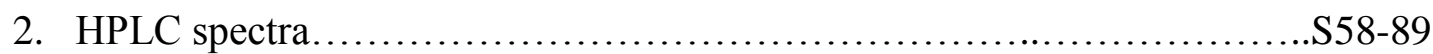


1. NMR spectra

(E)-methyl 2-((phenyl(o-tolyl)methylene)amino)acetate (1a)
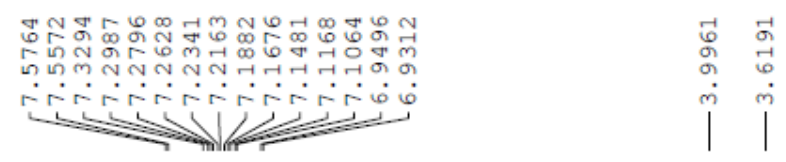

0
$\stackrel{0}{\circ}$
ơ
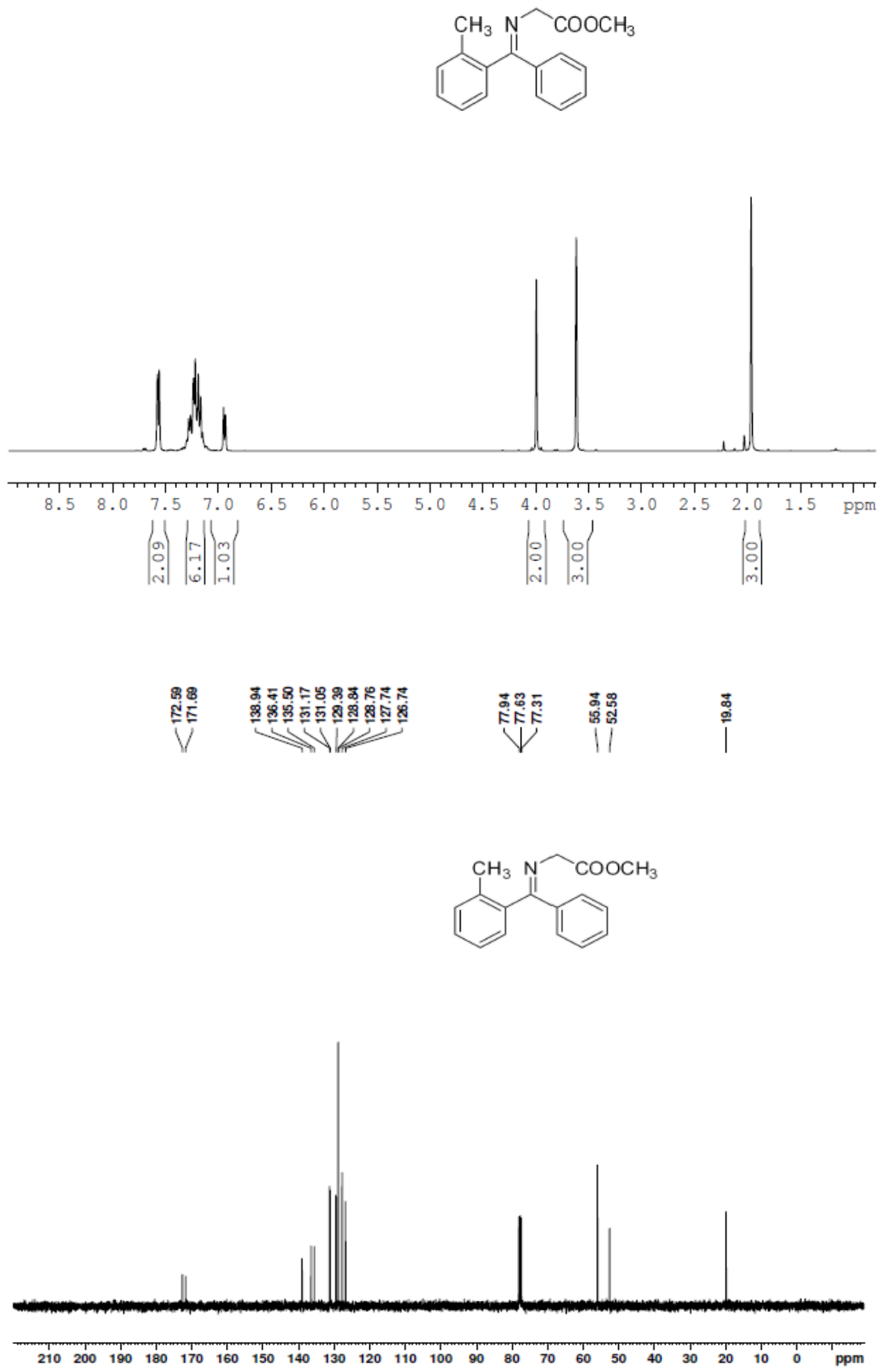

S2 
(E)-methyl 2-(((3-methoxyphenyl)(o-tolyl)methylene)amino)acetate (1b)
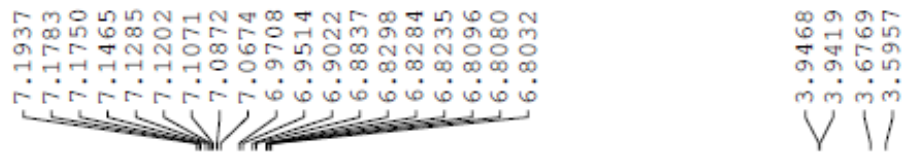<smiles>COC(=O)CN=C(c1cccc(OC)c1)c1ccccc1C</smiles>

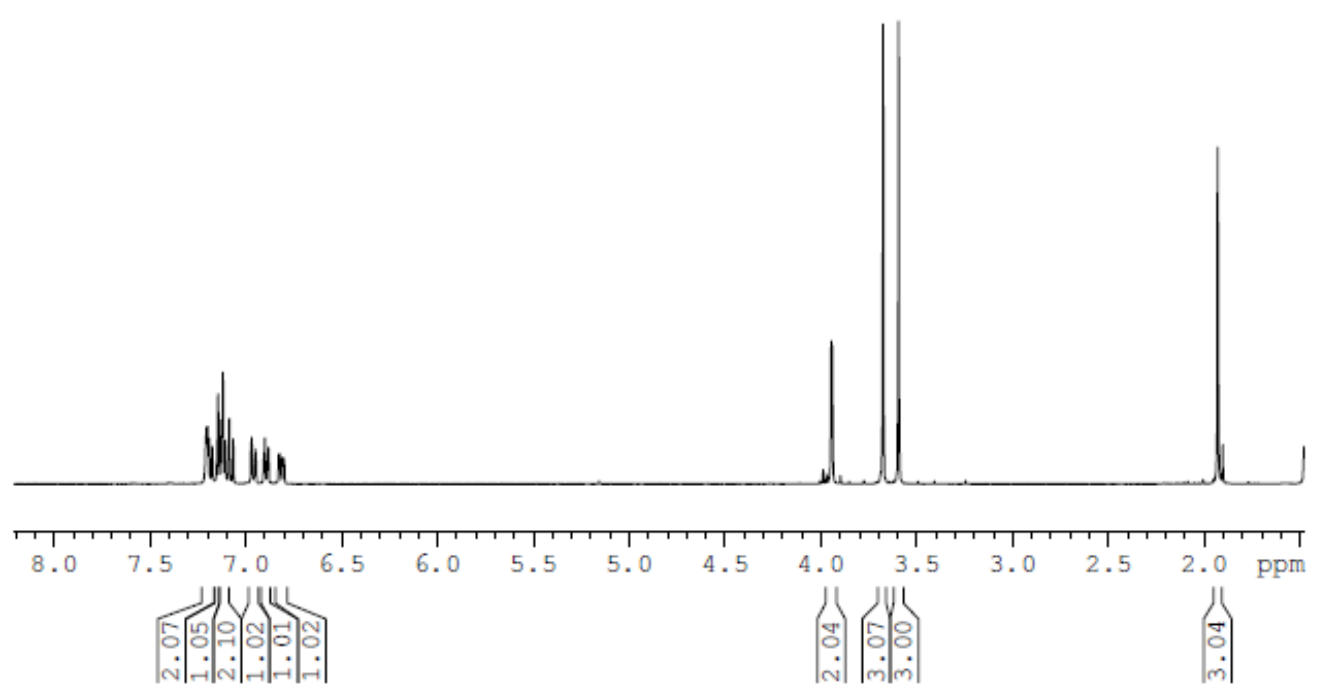

|

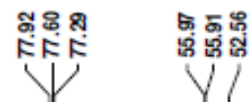

$\overbrace{\text { | }}^{\stackrel{\Phi}{\infty}}$<smiles>COC(=O)CN=C(c1cccc(OC)c1)c1ccccc1C</smiles>

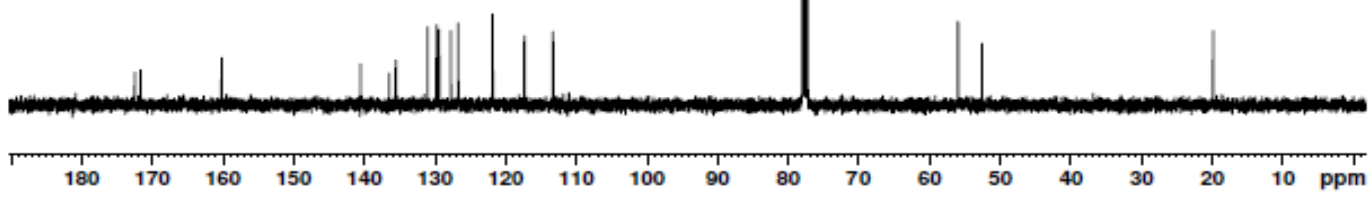


(E)-methyl 2-((m-tolyl $(o$-tolyl)methylene)amino)acetate (1c)
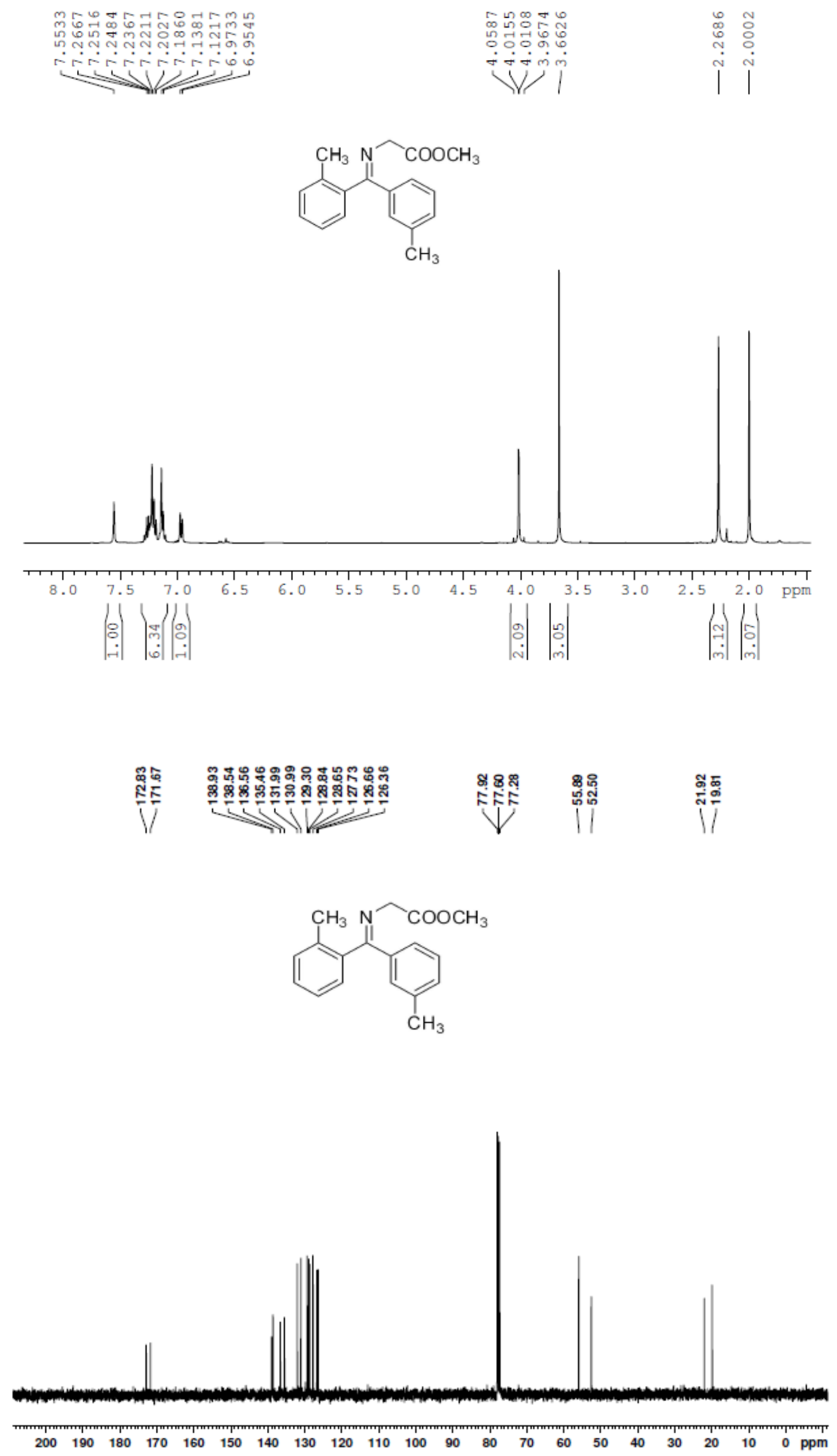
(E)-methyl 2-((o-tolyl(p-tolyl)methylene)amino)acetate (1d)
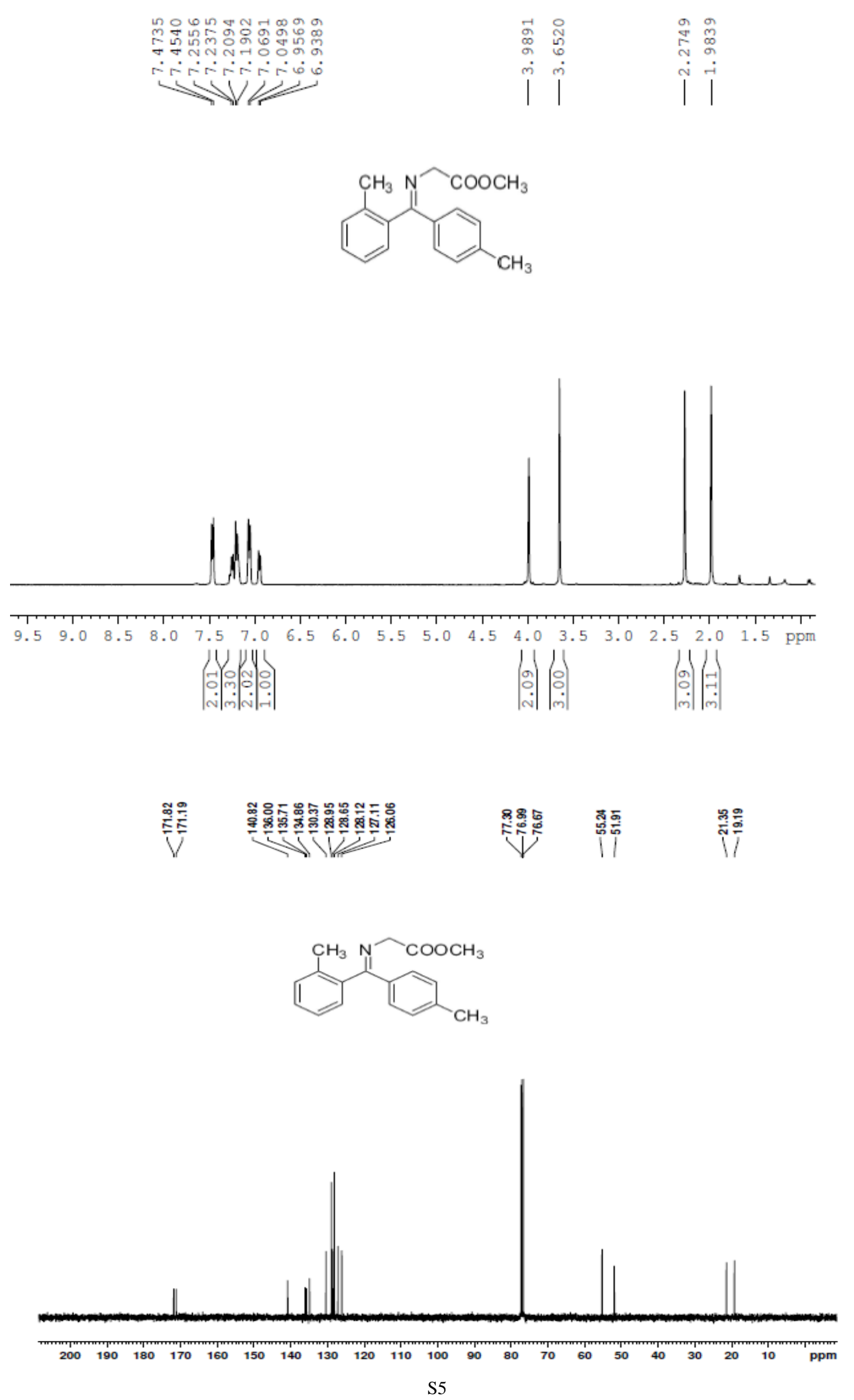
(E)-methyl 2-(((4-fluorophenyl)(o-tolyl)methylene)amino)acetate (1e)

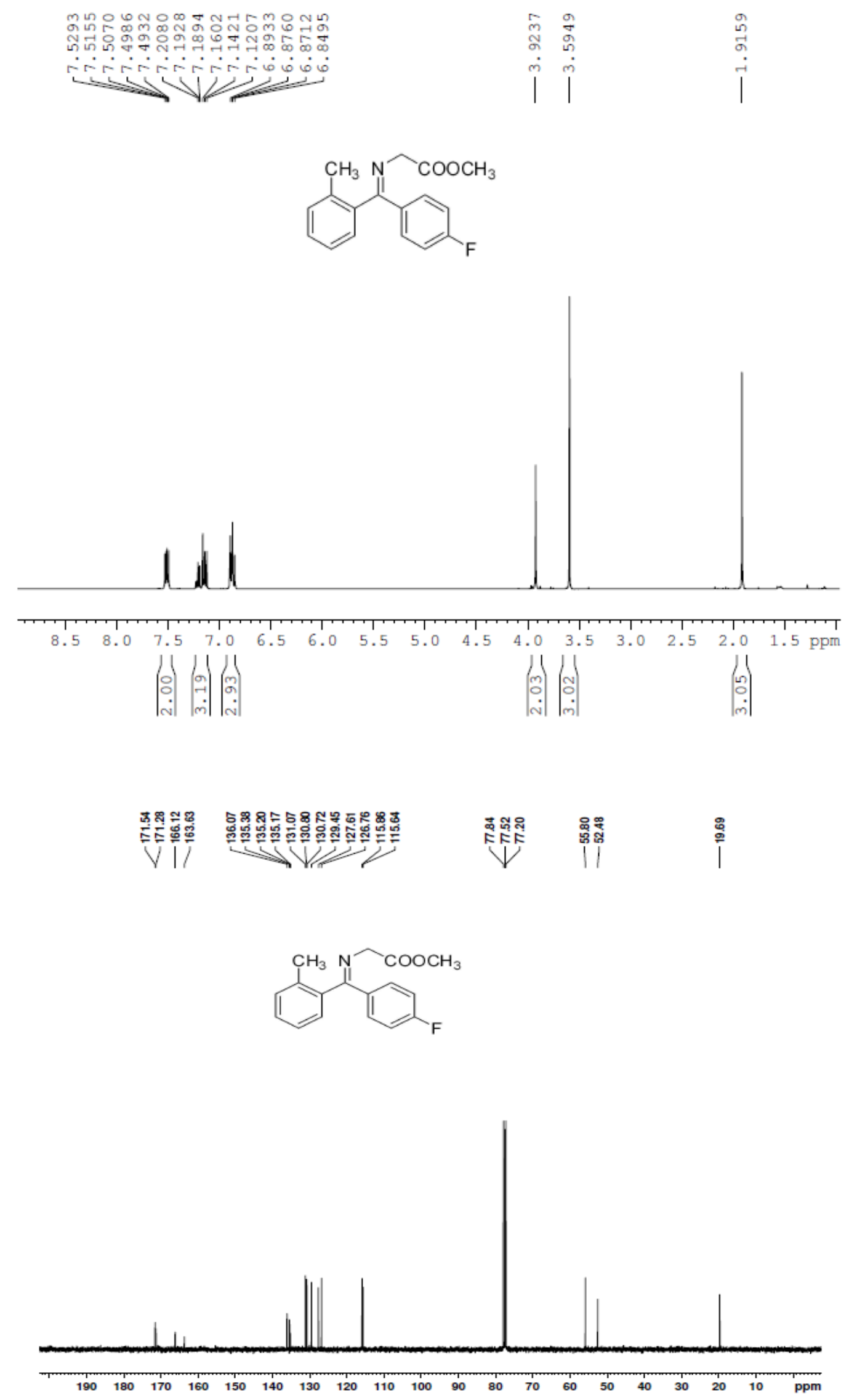


(E)-methyl 2-(((2-methoxyphenyl)(phenyl)methylene)amino)acetate (1f)

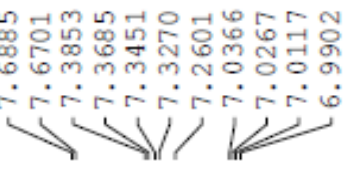

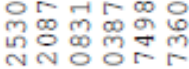

.

$11 / V$<smiles>COC(=O)C/N=C(/c1ccccc1)c1ccccc1OC</smiles>

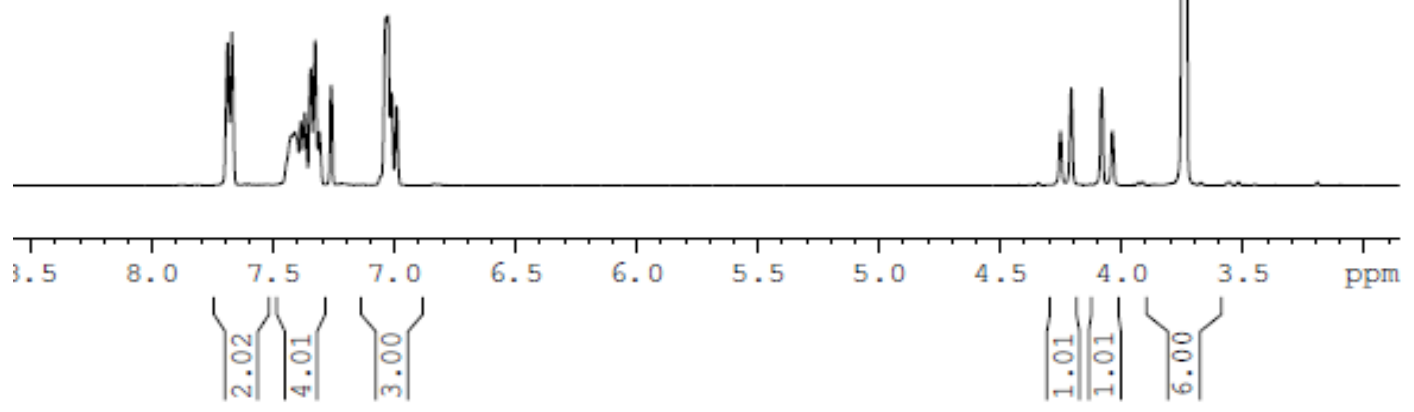

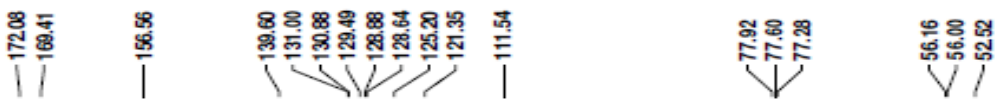<smiles>COC(=O)C/N=C(/c1ccccc1)c1ccccc1OC</smiles>

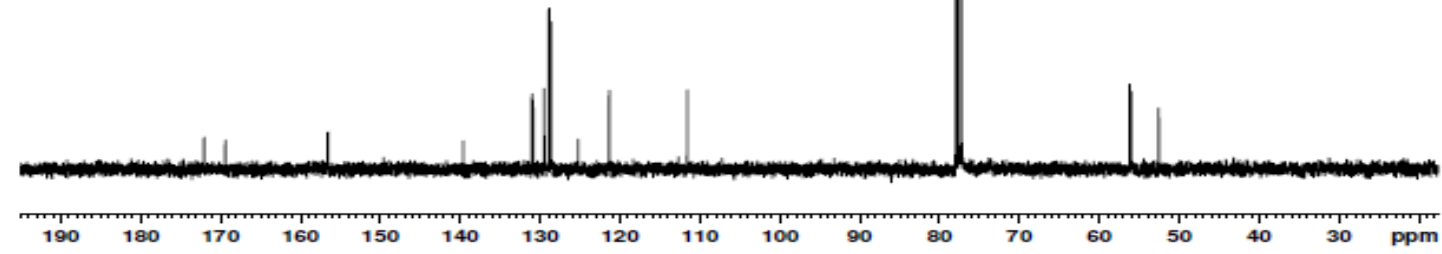


(E)-methyl 2-(((2-chlorophenyl)(phenyl)methylene)amino)acetate (1g)
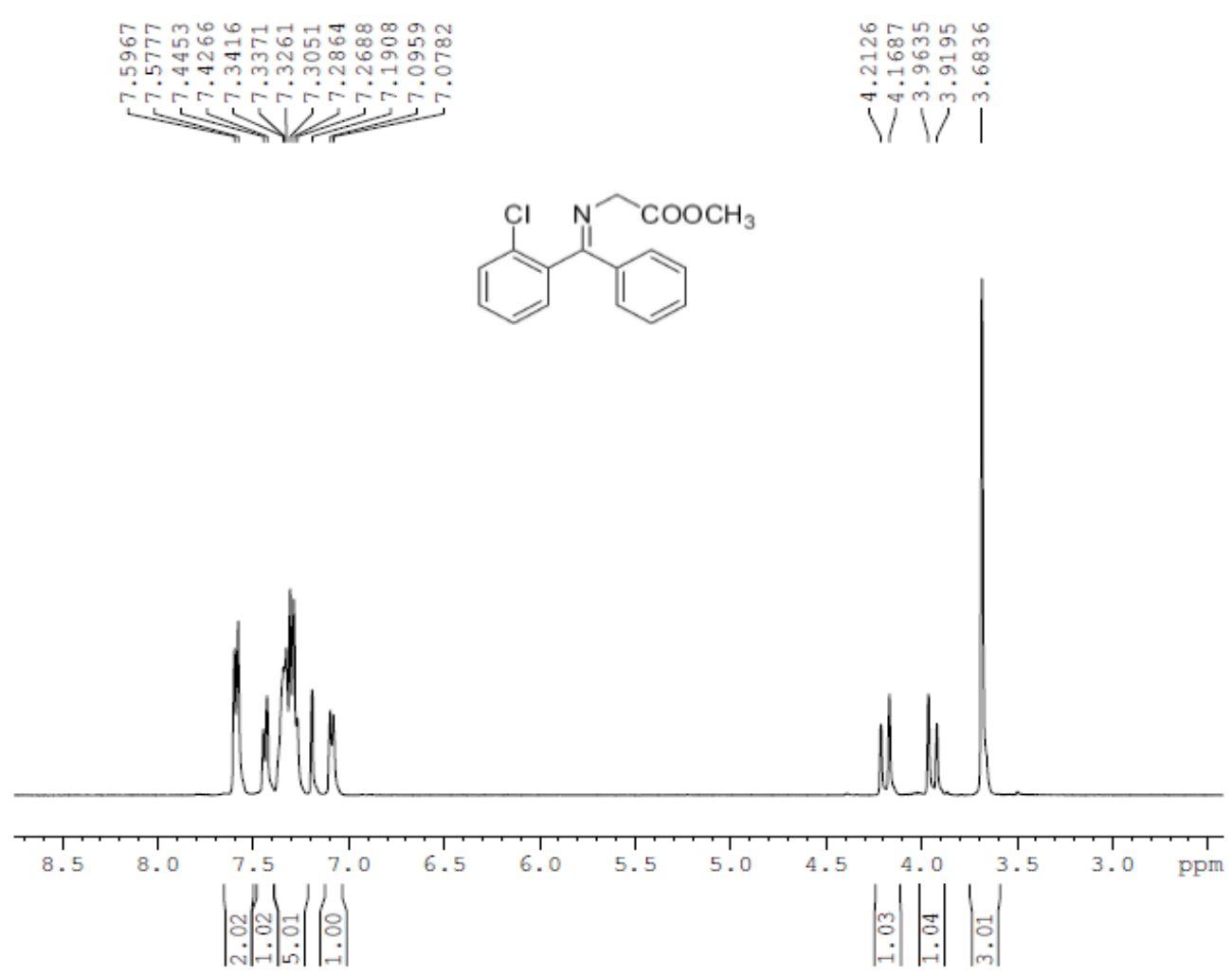

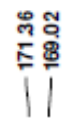

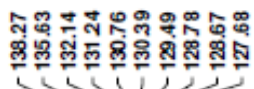

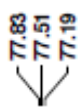

$\mid$
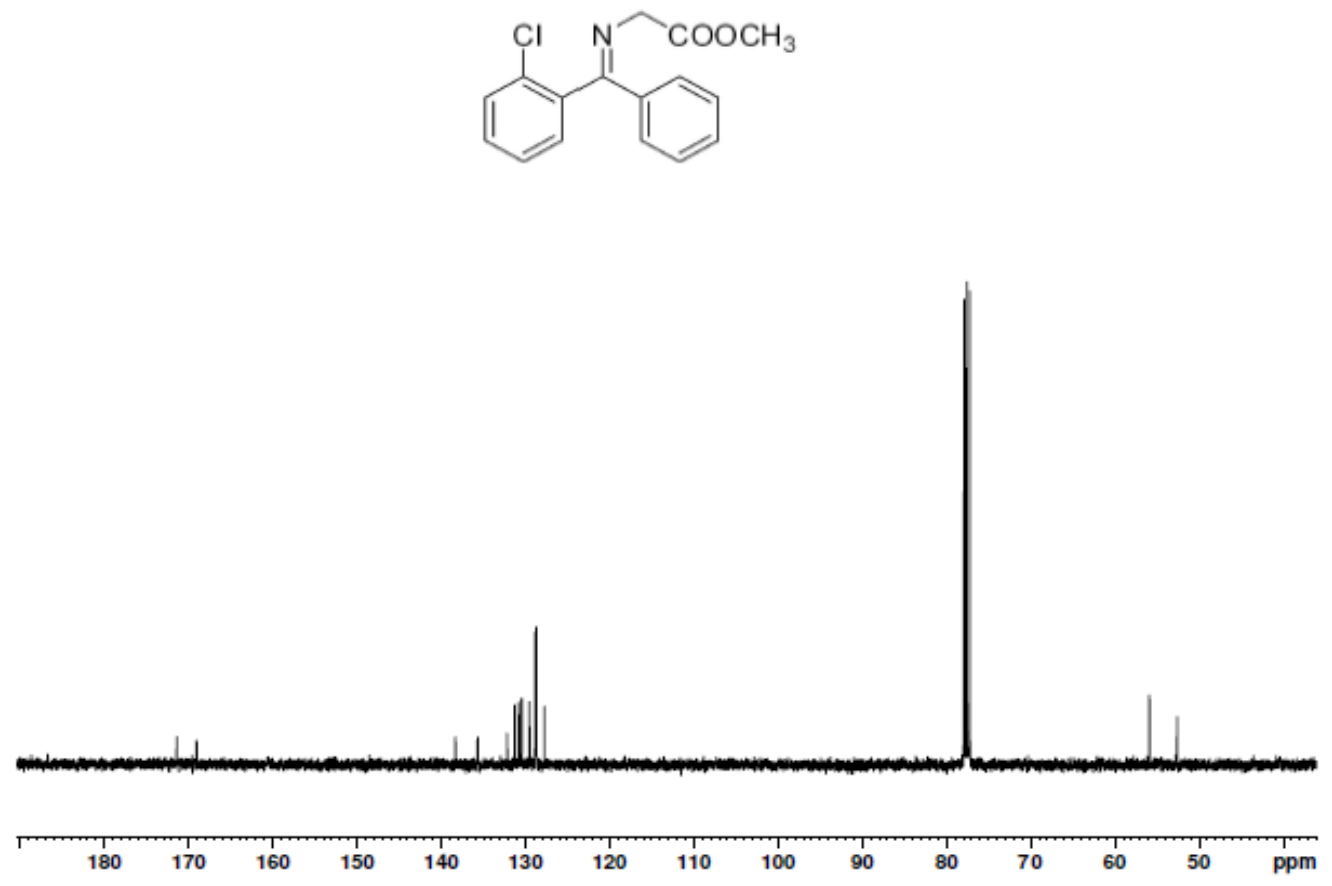

S8 
(E)-methyl 2-(((2-chlorophenyl)(3-methoxyphenyl)methylene)amino)acetate (1h)

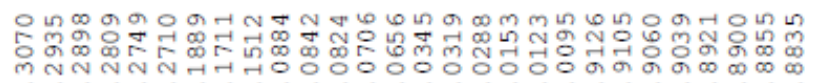

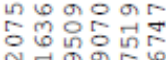

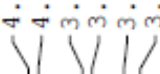<smiles>COC(=O)CN=C(c1cccc(OC)c1)c1ccccc1Cl</smiles>

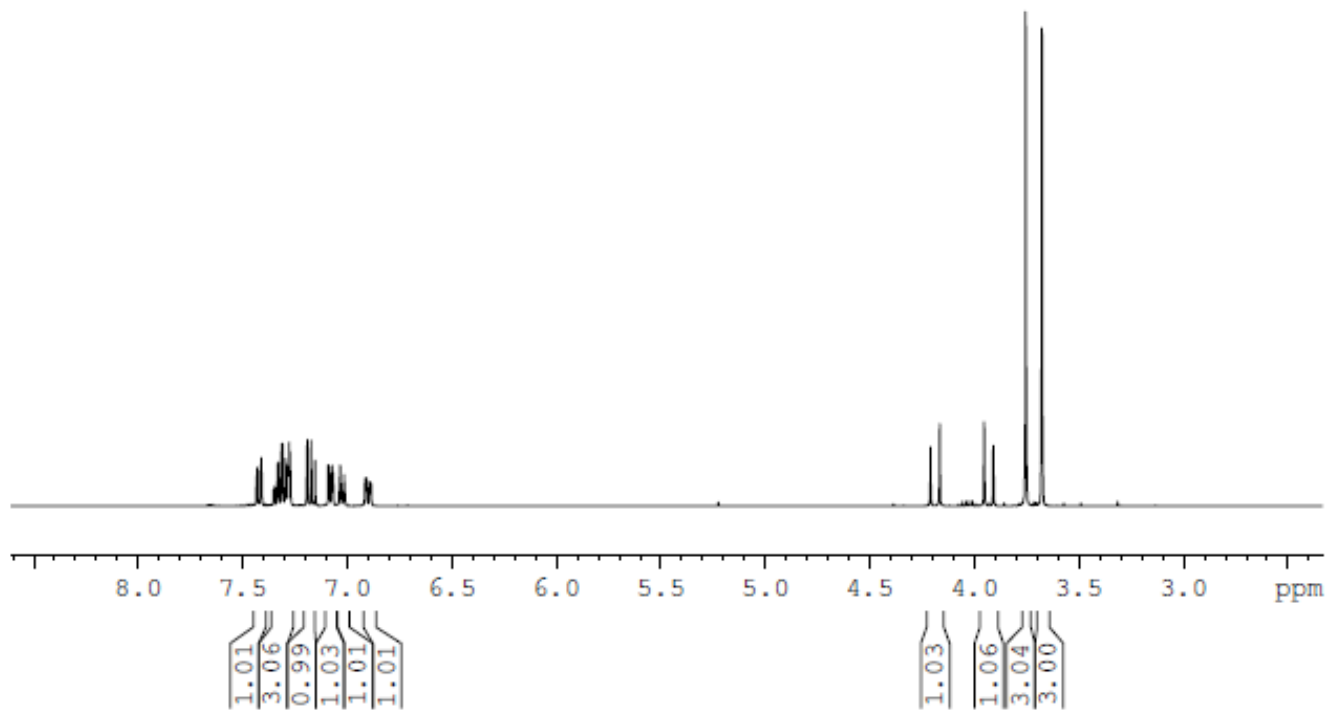

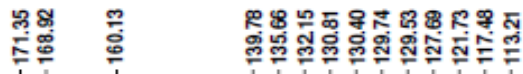

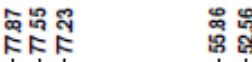

Tा

Noil

V

|<smiles>COC(=O)CN=C(c1cccc(OC)c1)c1ccccc1Cl</smiles>

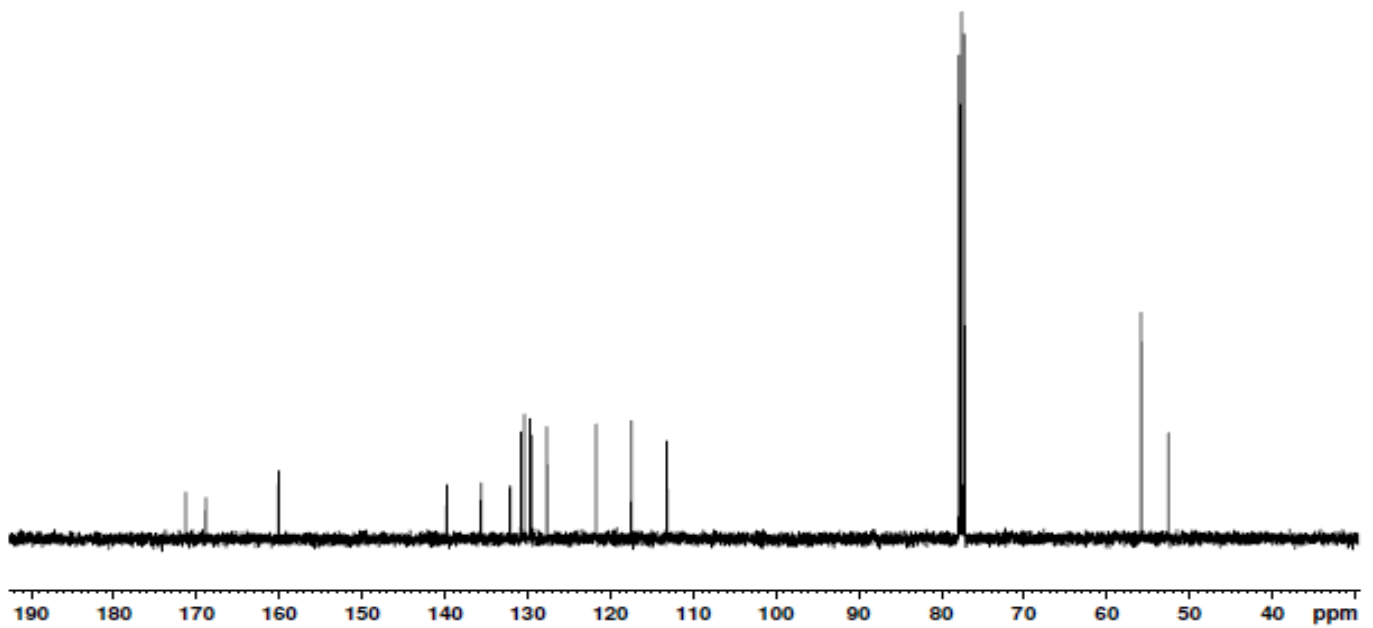


(E)-methyl 2-(((2-chlorophenyl)(m-tolyl)methylene)amino)acetate (1i)
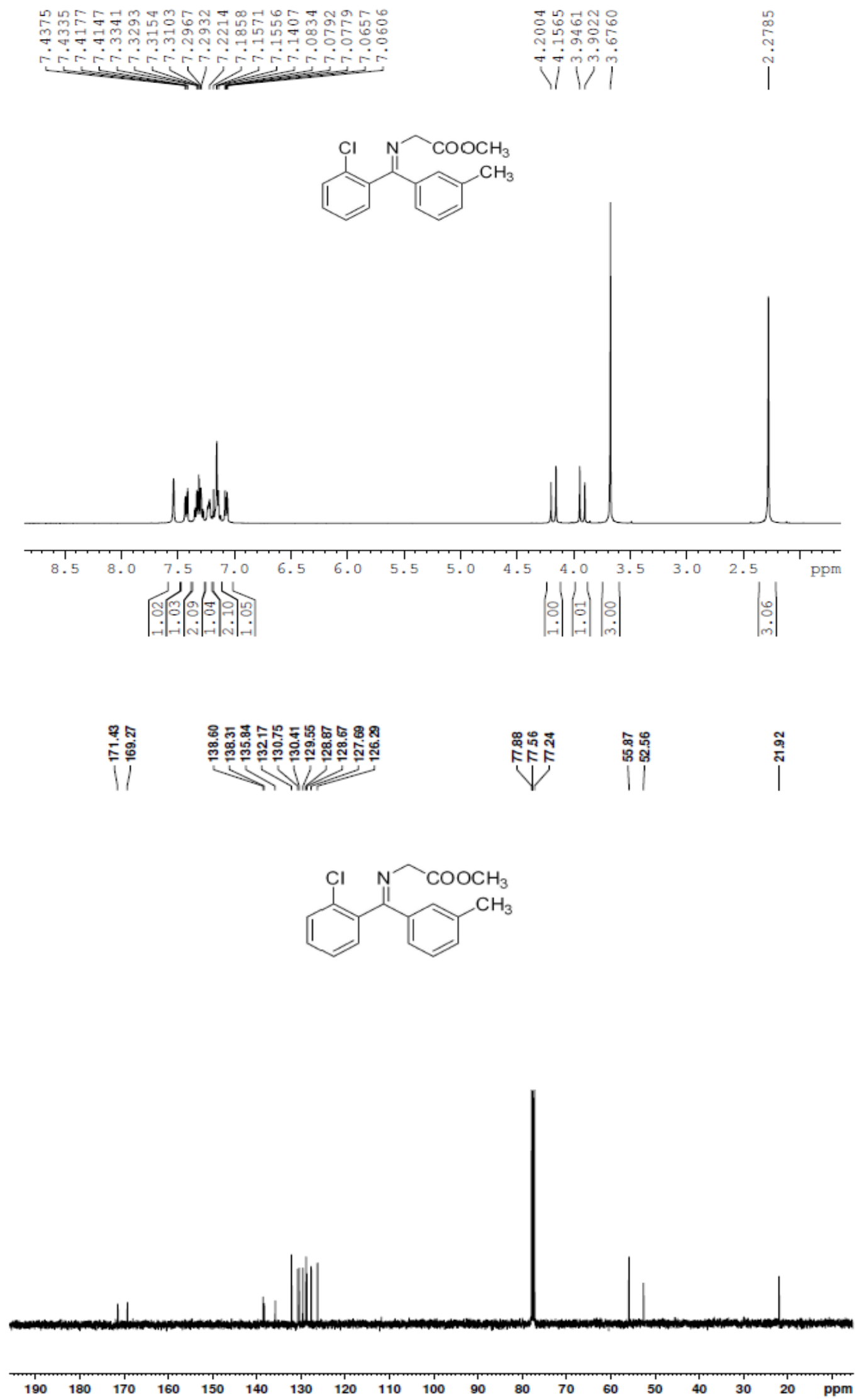
(E)-methyl 2-(((2-chlorophenyl)(p-tolyl)methylene)amino)acetate (1j)
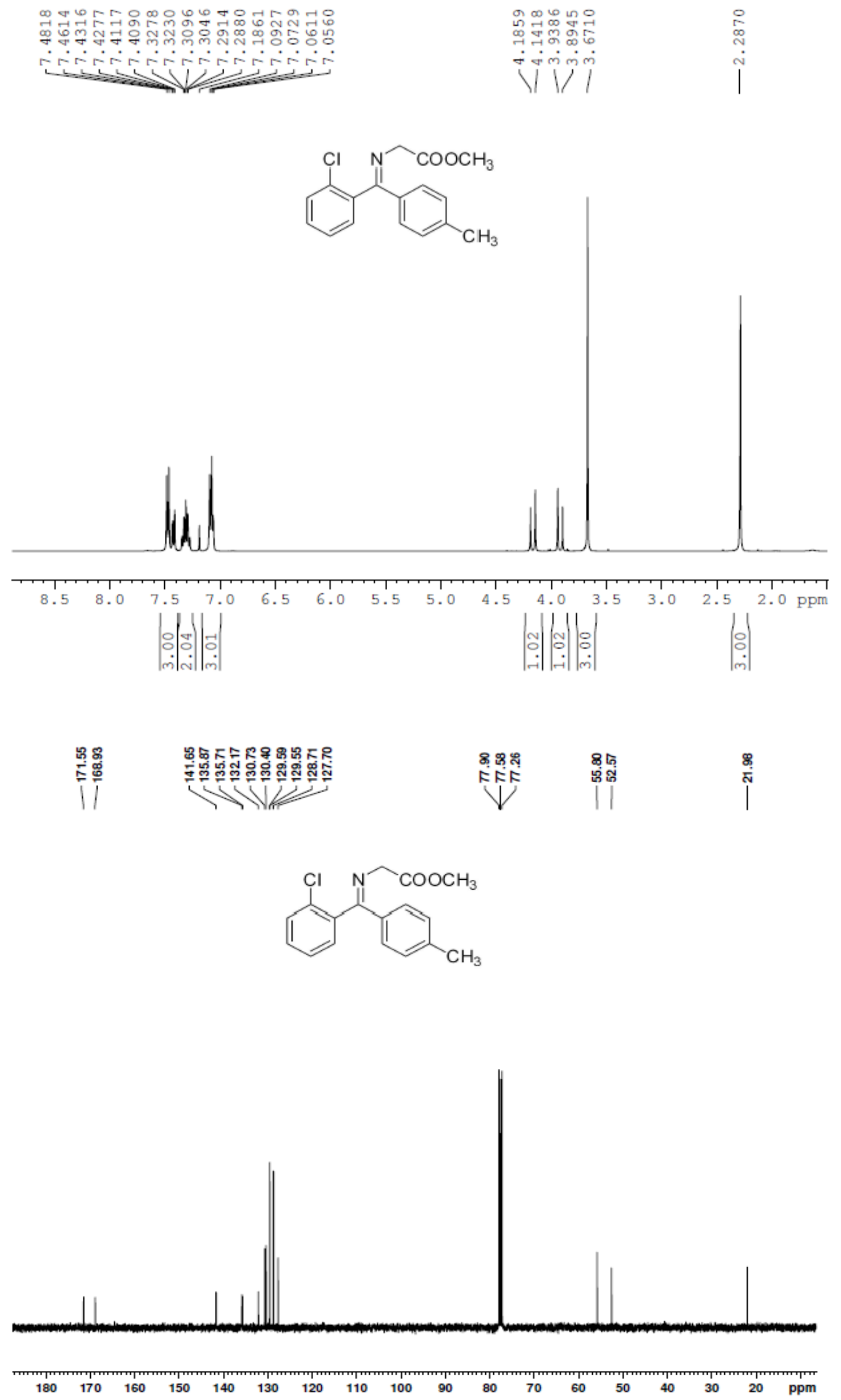
(E)-methyl 2-(((2-chlorophenyl)(4-fluorophenyl)methylene)amino)acetate (1k)

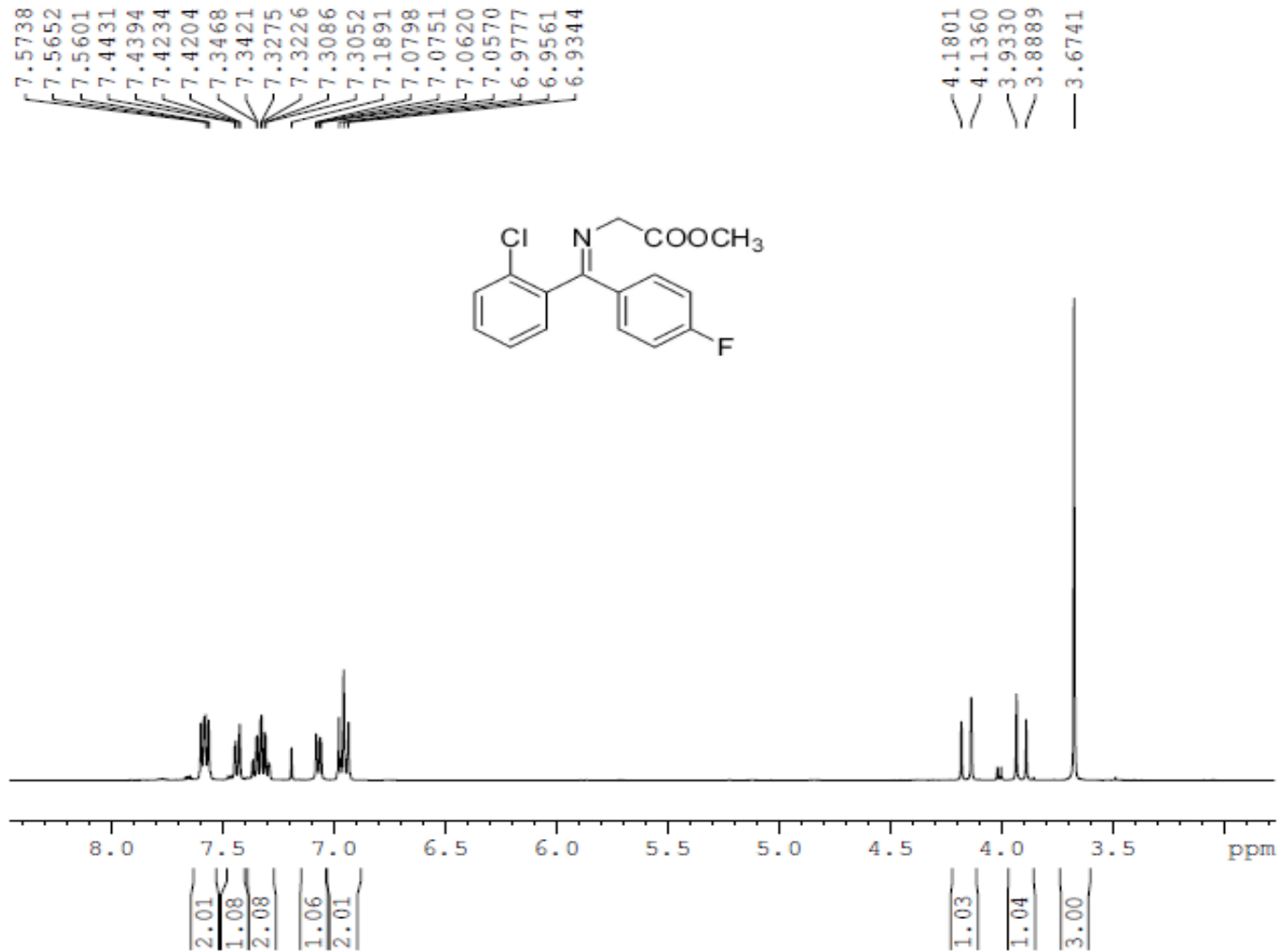

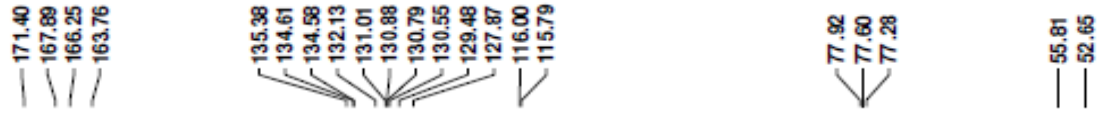<smiles>CC(=O)CN=C(c1ccc(F)cc1)c1ccccc1Cl</smiles>
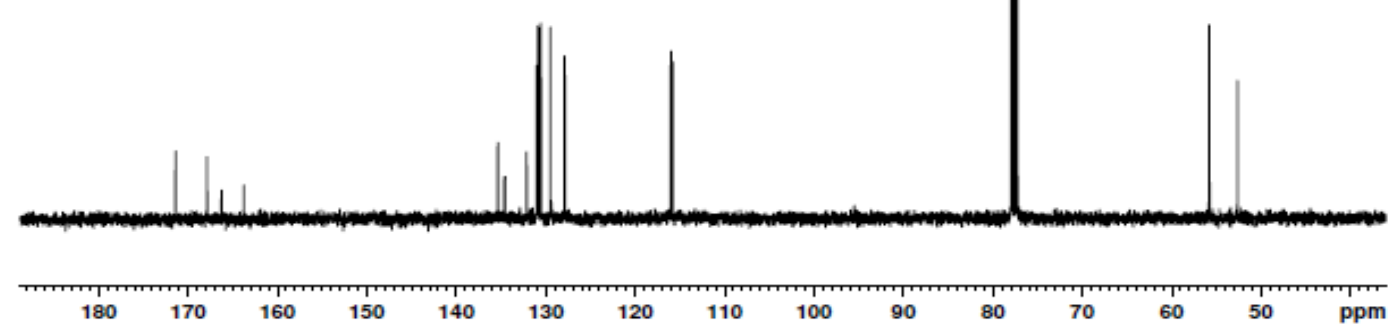
(E)-methyl 2-(((2-chlorophenyl)(4-chlorophenyl)methylene)amino)acetate (11)

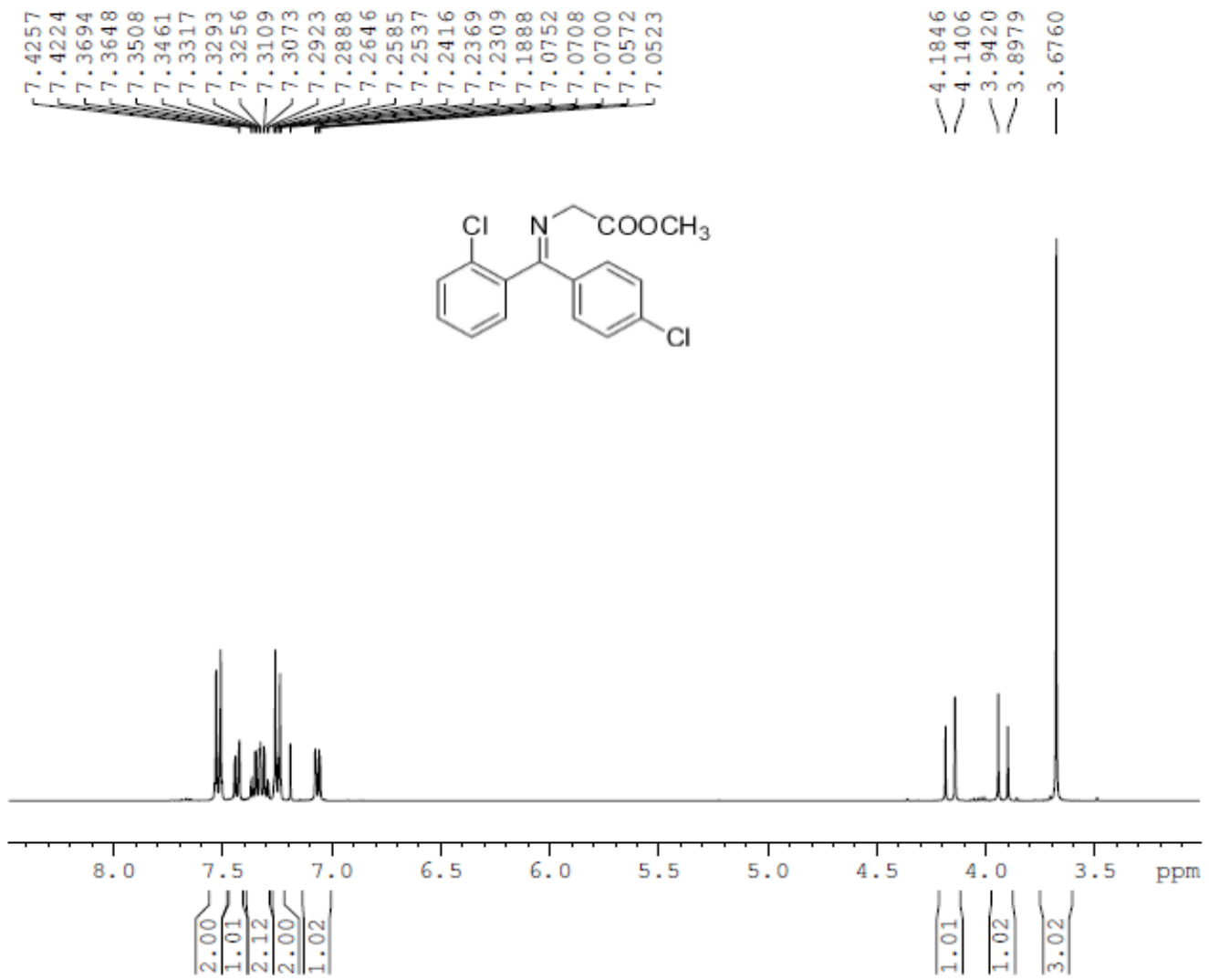

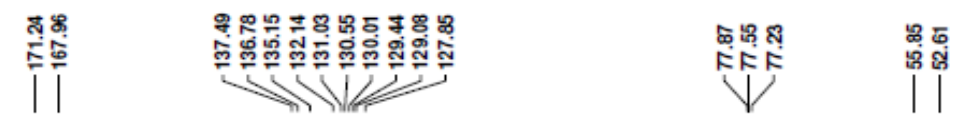<smiles>CC(=O)CN=C(c1ccc(Cl)cc1)c1ccccc1Cl</smiles>

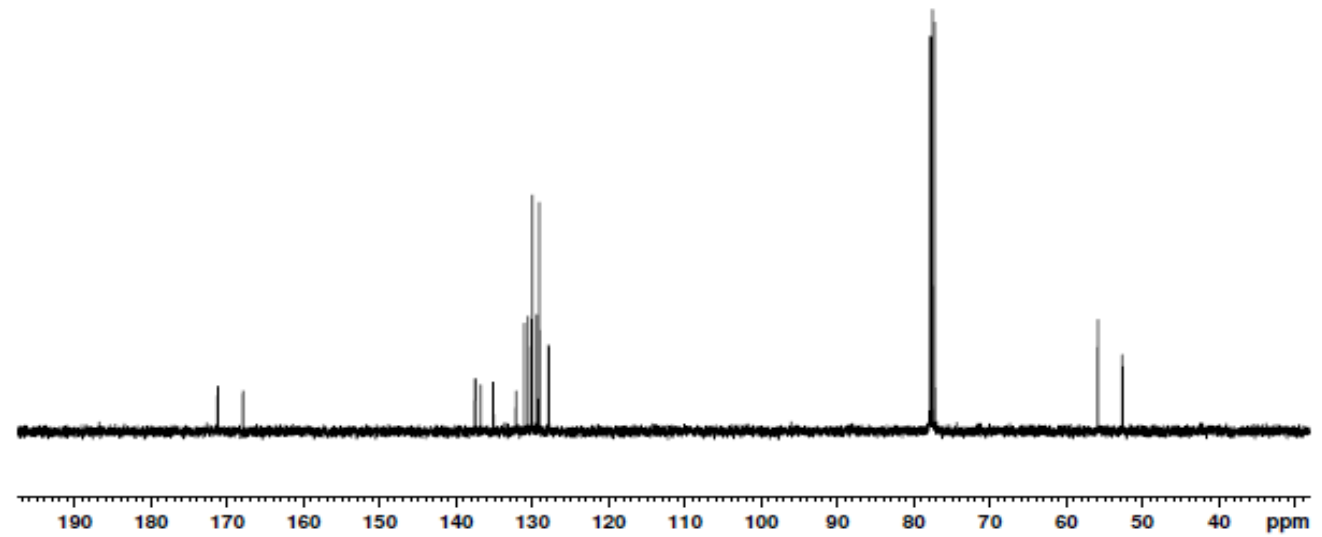


(E)-methyl 2-(((2-bromophenyl)(phenyl)methylene)amino)acetate (1m)
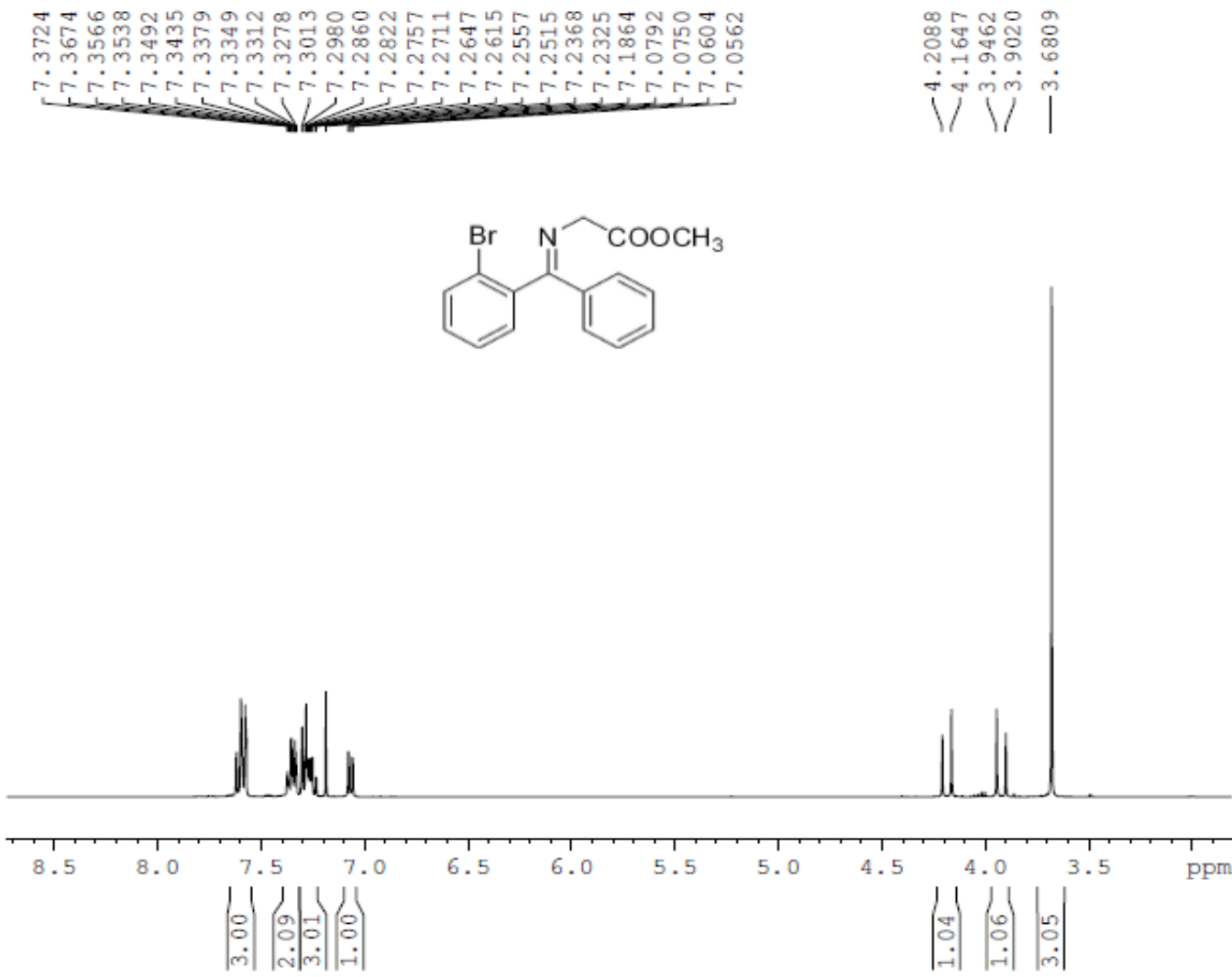

욤웅

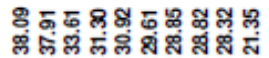

-

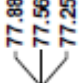

密

T⿱一⿻上丨

|<smiles>COC(=O)CN=C(c1ccccc1)c1ccccc1Br</smiles>
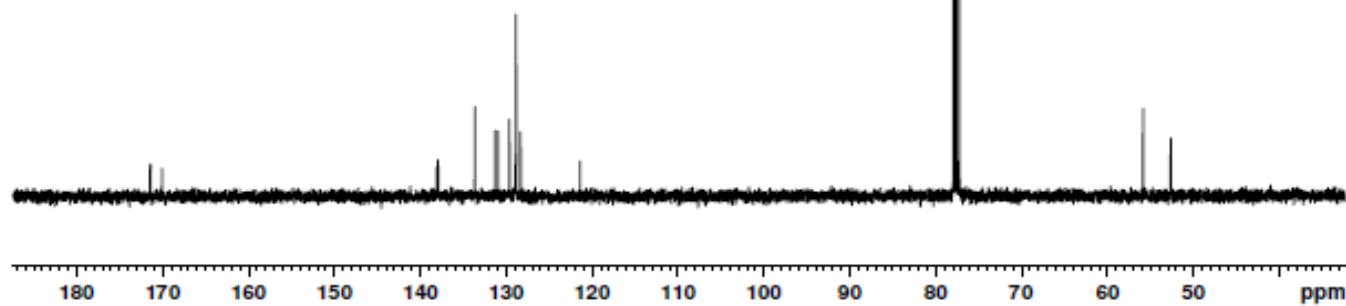

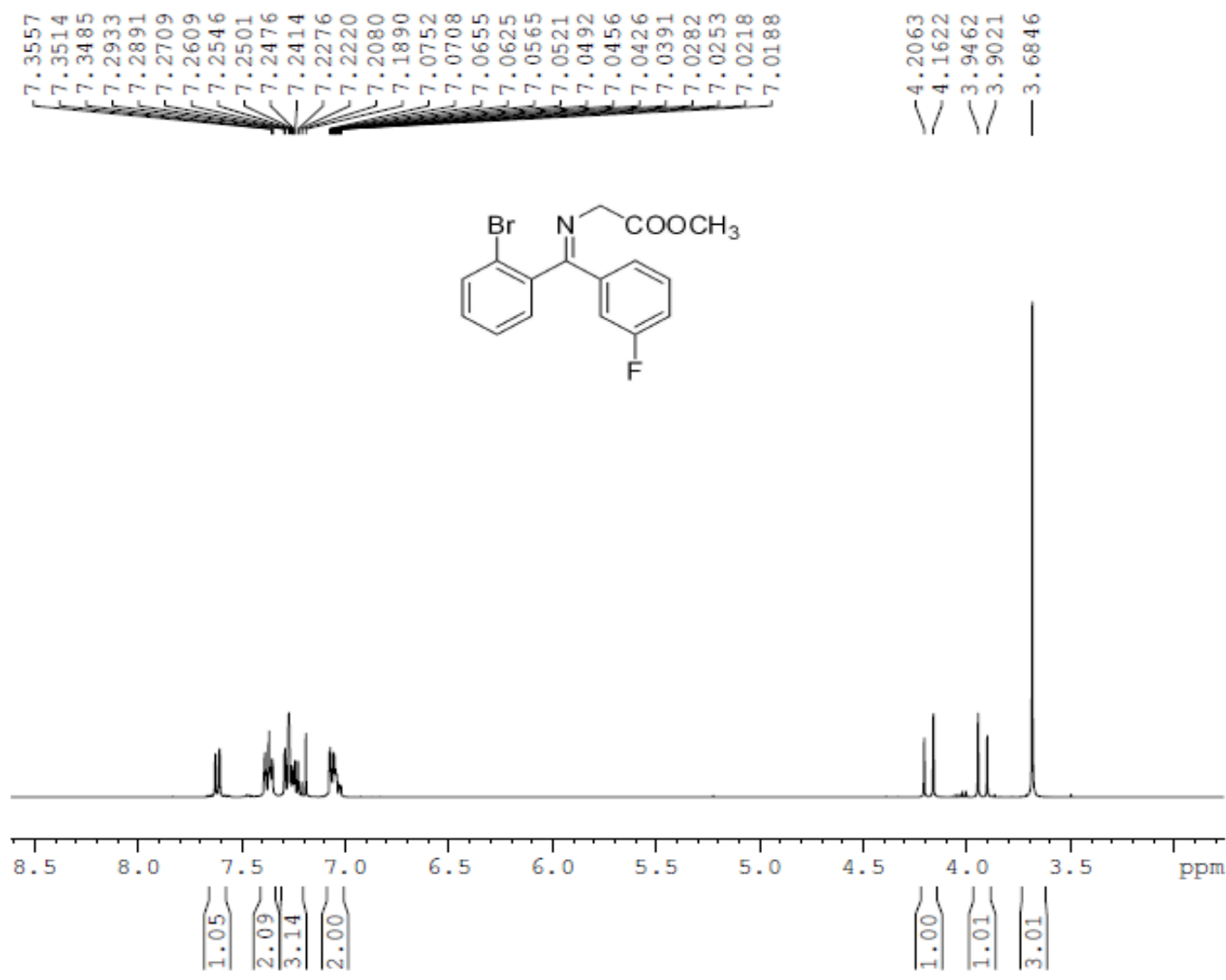

||||<smiles>CC(=O)CN=C(c1cccc(F)c1)c1ccccc1Br</smiles>

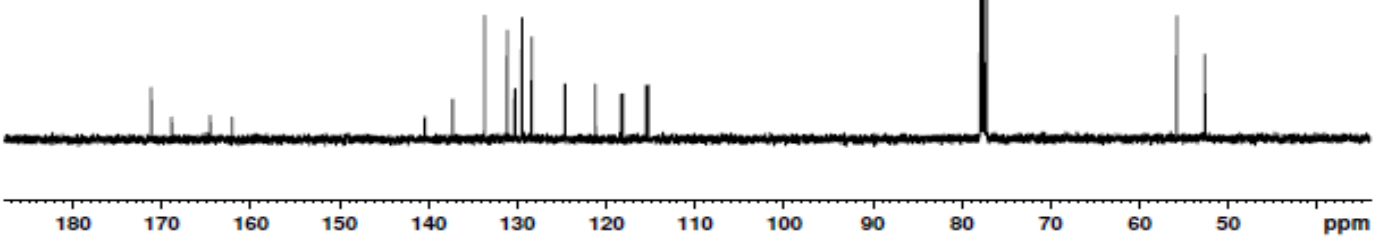


(E)-methyl 2-(((2-bromophenyl)(4-fluorophenyl)methylene)amino)acetate (10)
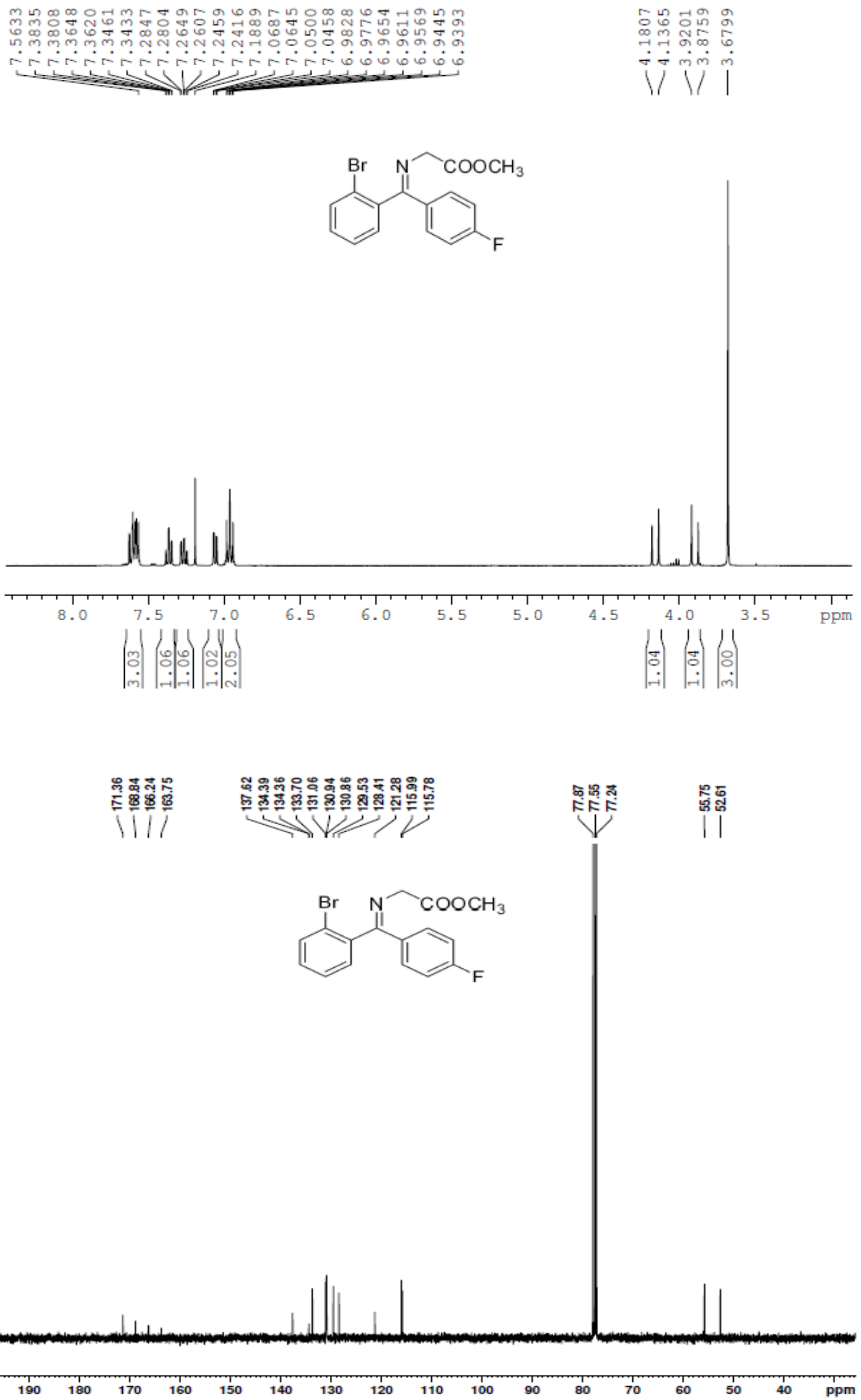
(E)-methyl 2-(((2-bromophenyl)(4-chlorophenyl)methylene)amino)acetate (1p)
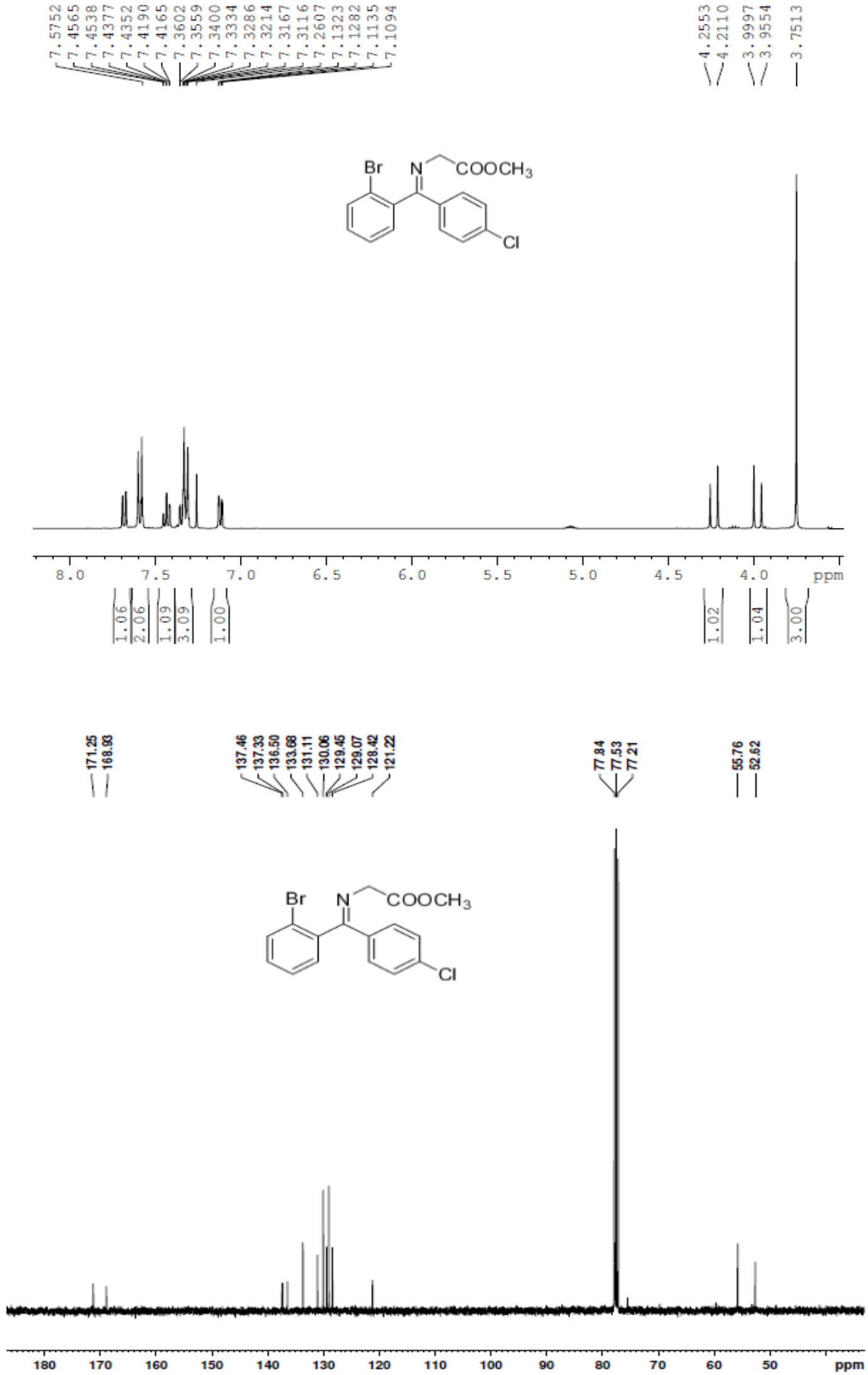
(E)-methyl 2-(((2-fluorophenyl)(phenyl)methylene)amino)acetate (1q)
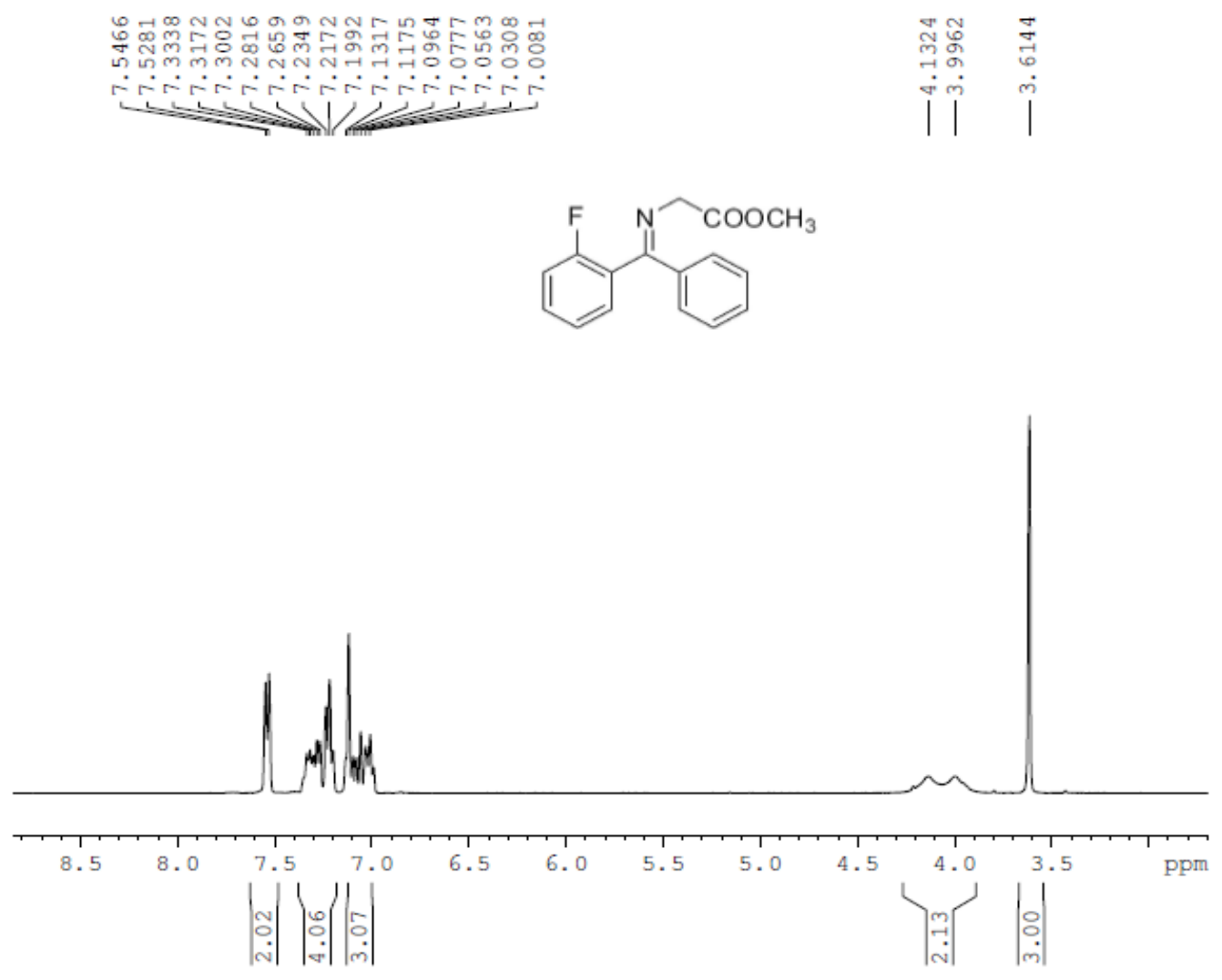

筩

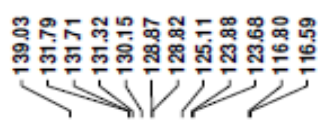

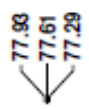

葪
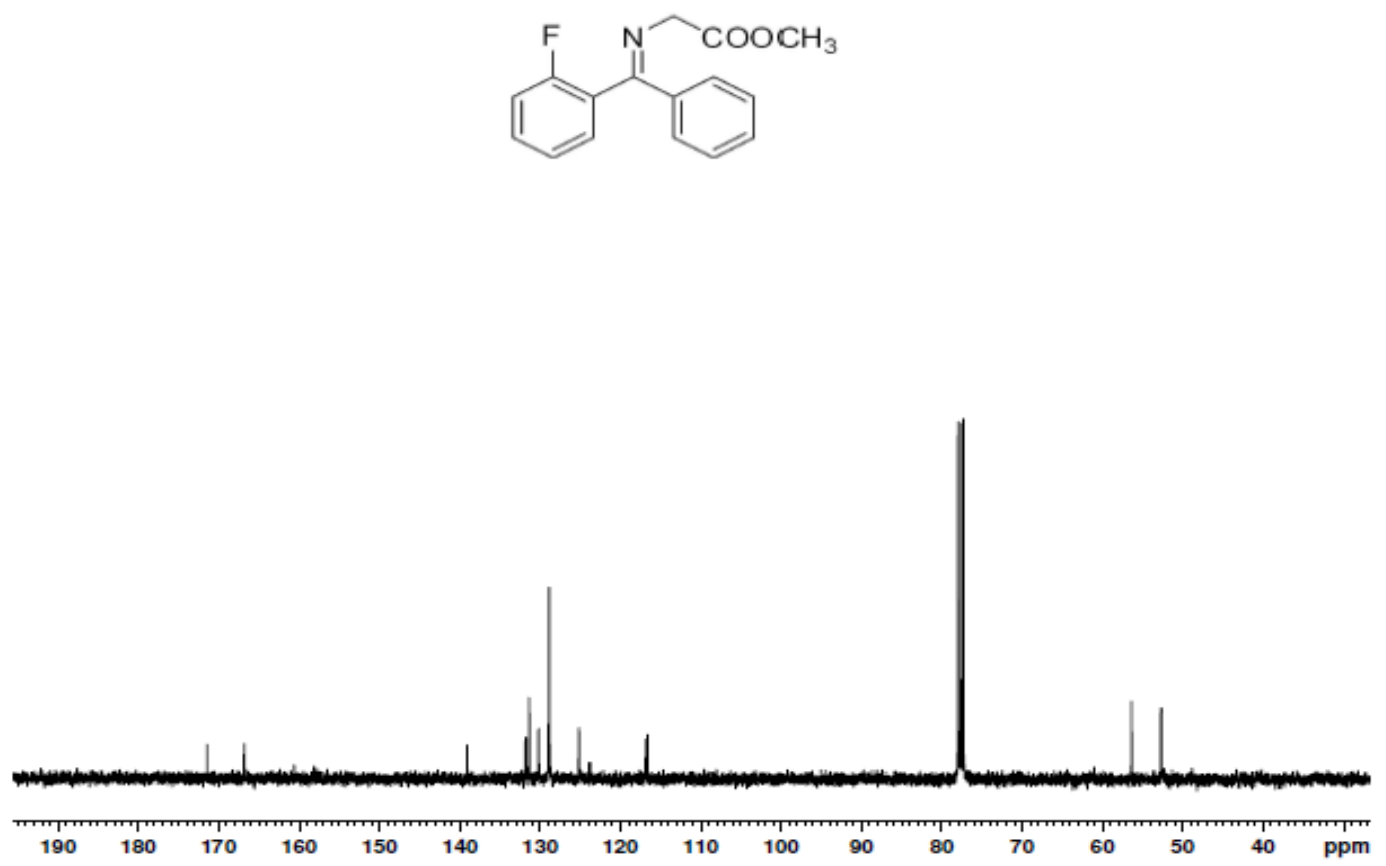

S18 
(E)-methyl 2-(((2-fluorophenyl)(m-tolyl)methylene)amino)acetate (1r)

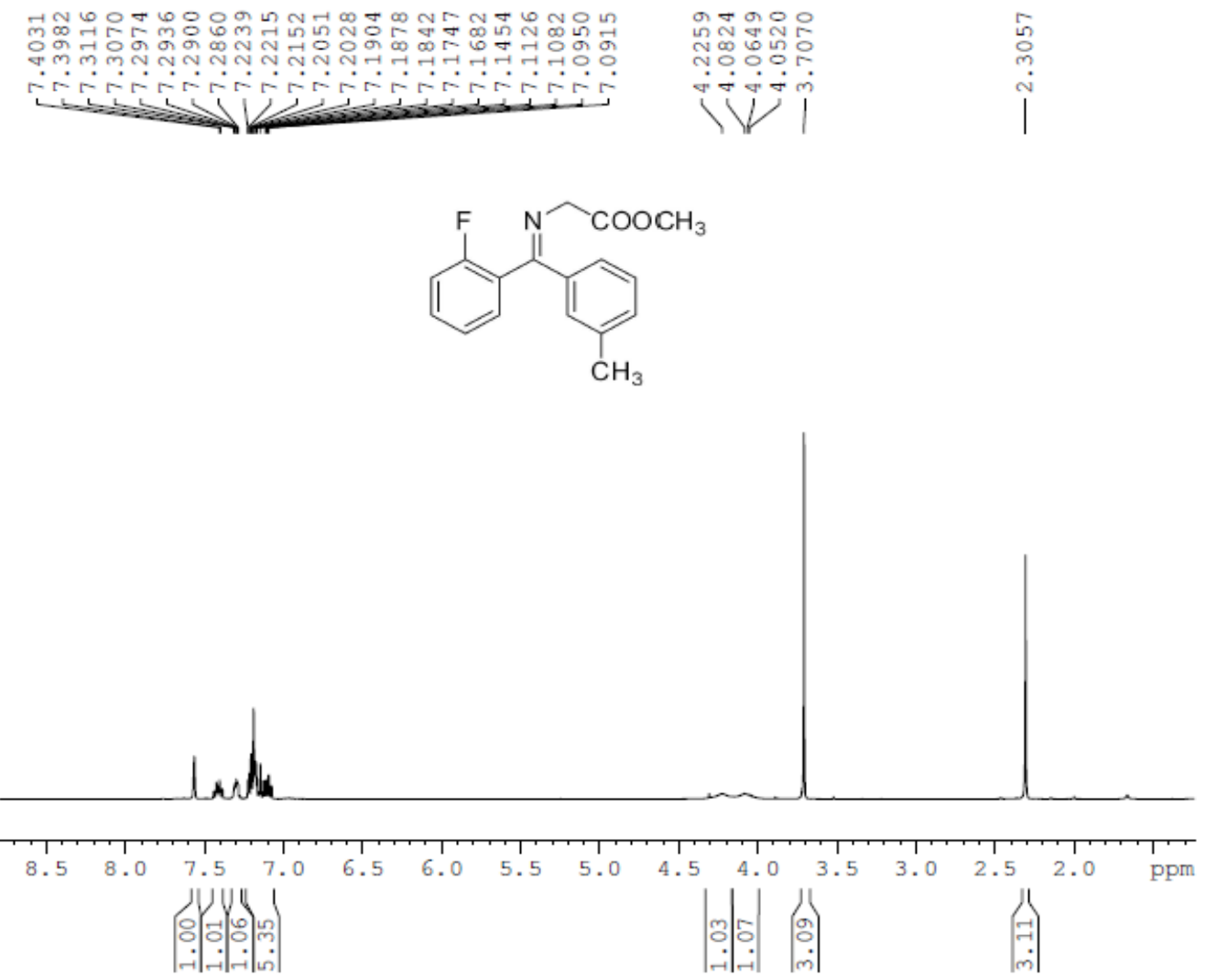

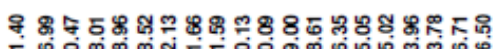
토웅

$1 \mid 111$

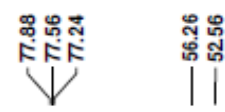

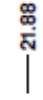

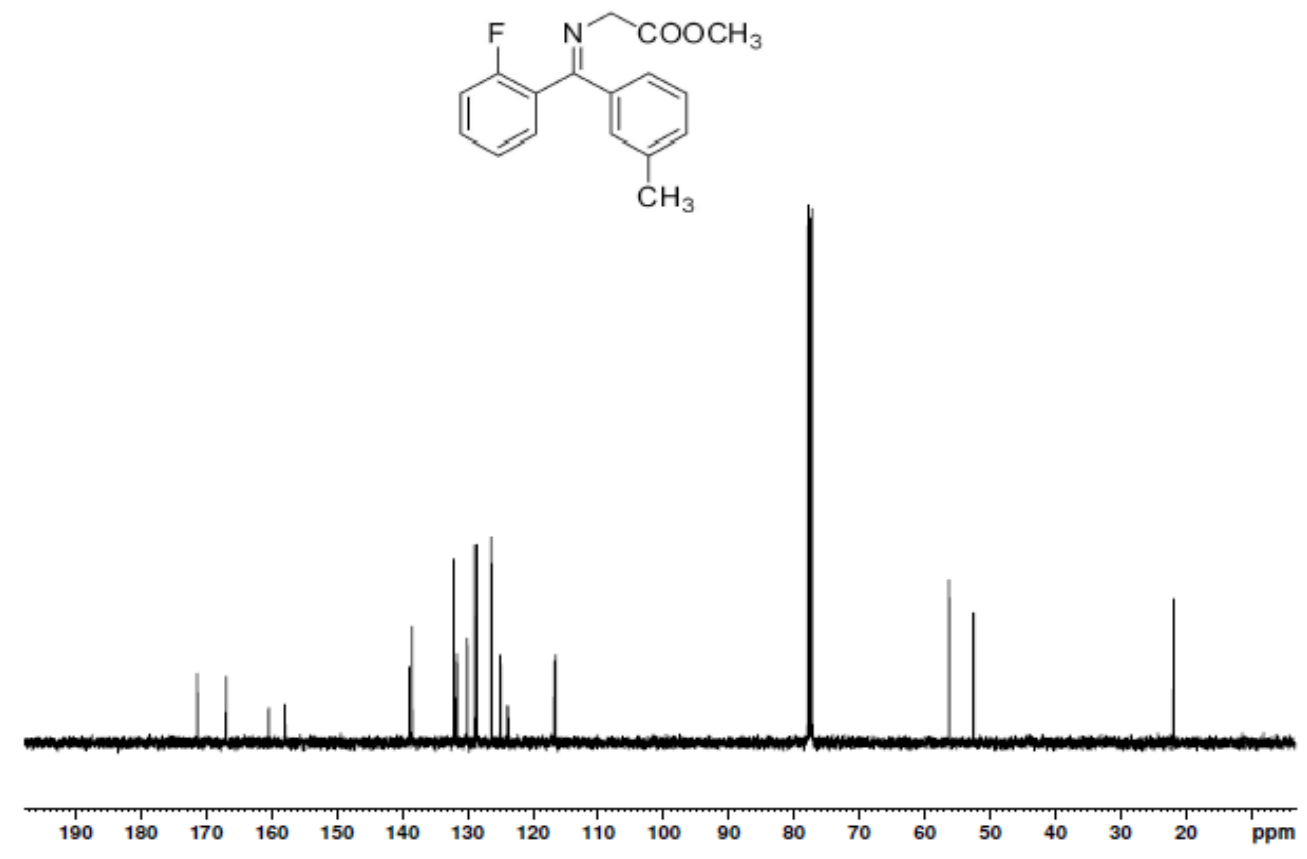


(E)-methyl 2-(((2-fluorophenyl)(4-fluorophenyl)methylene)amino)acetate (1s)
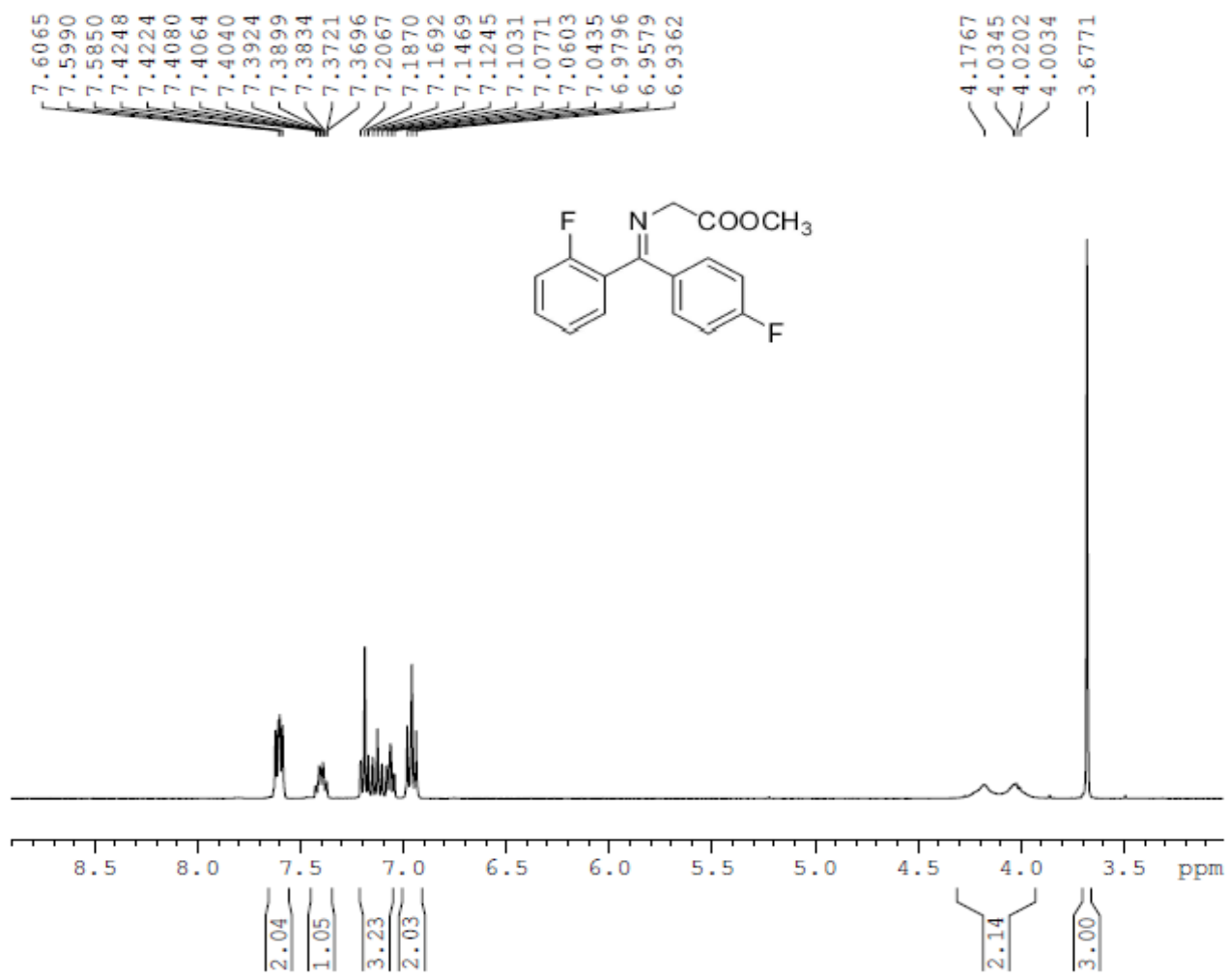

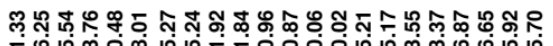
E.

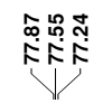

สู่

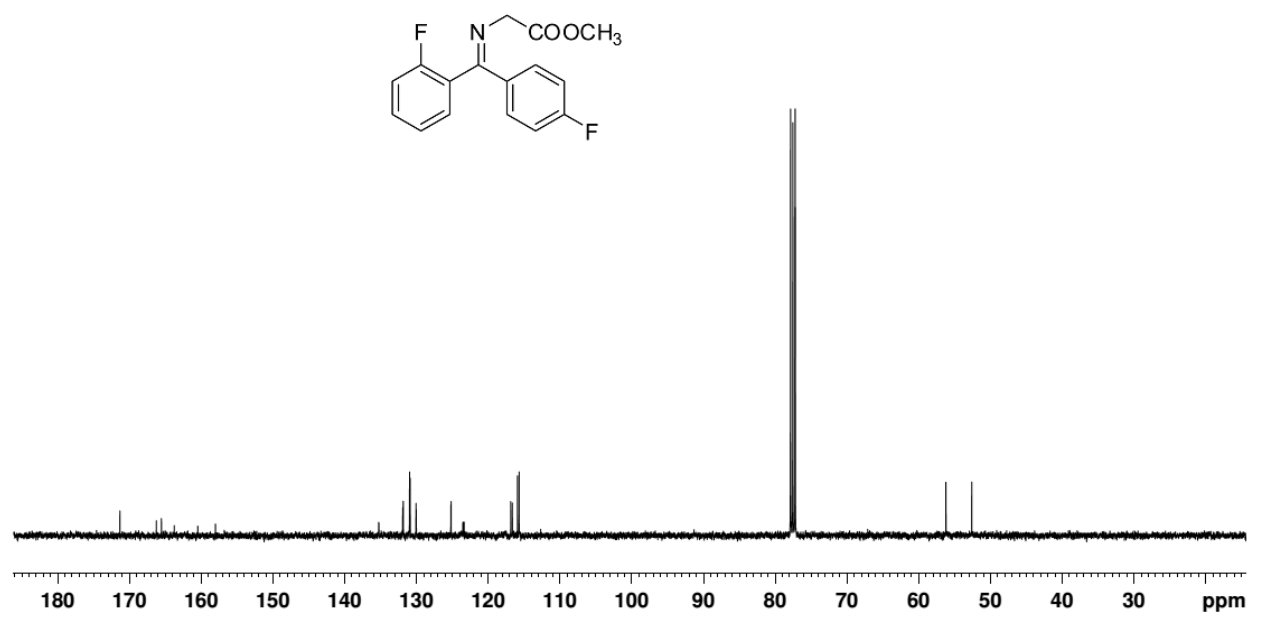

S20 
(E)-methyl 2-((naphthalen-1-yl(phenyl)methylene)amino)acetate (1t)
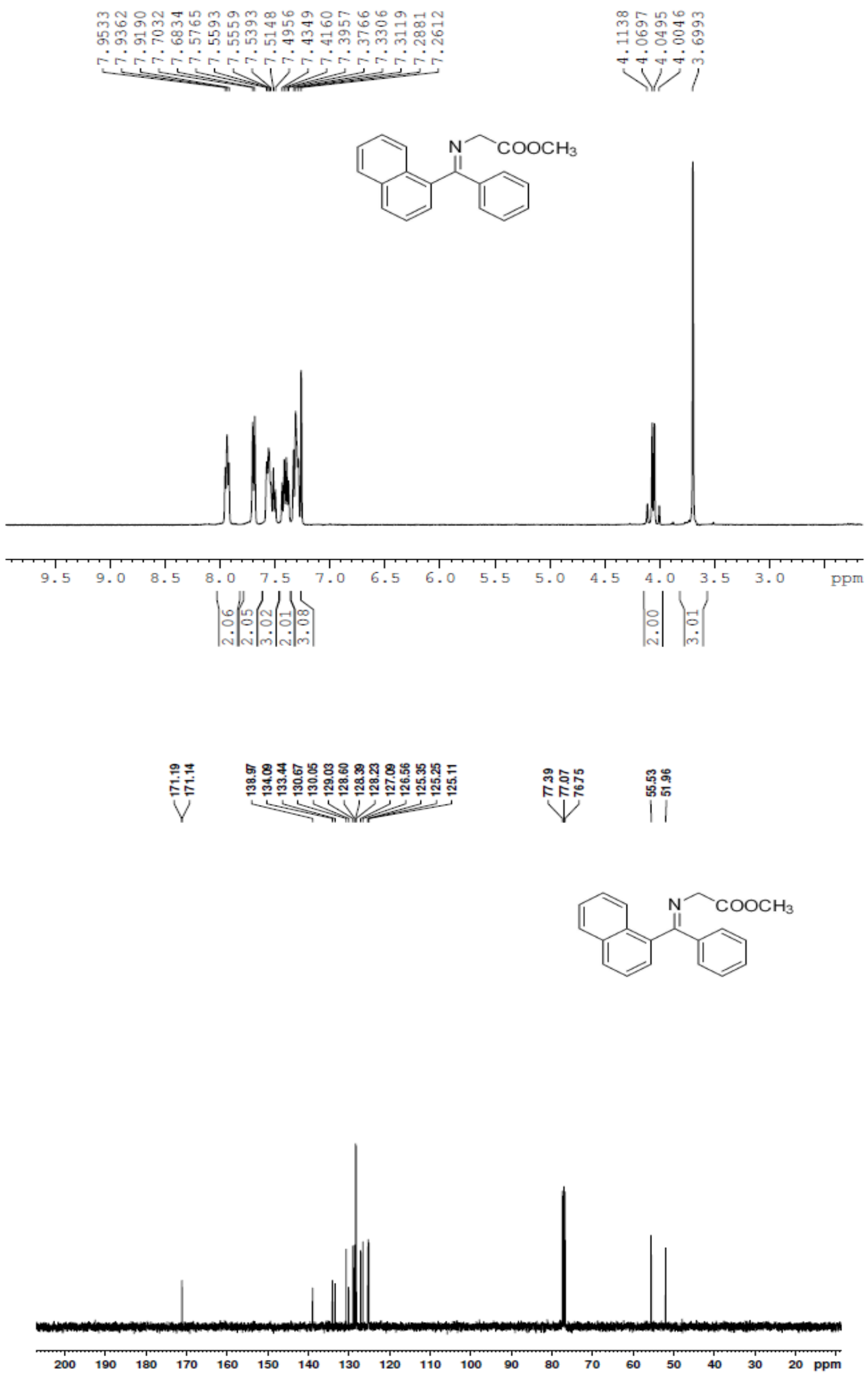

S21 
(E)-ethyl 2-((phenyl(o-tolyl)methylene)amino)acetate (1u)
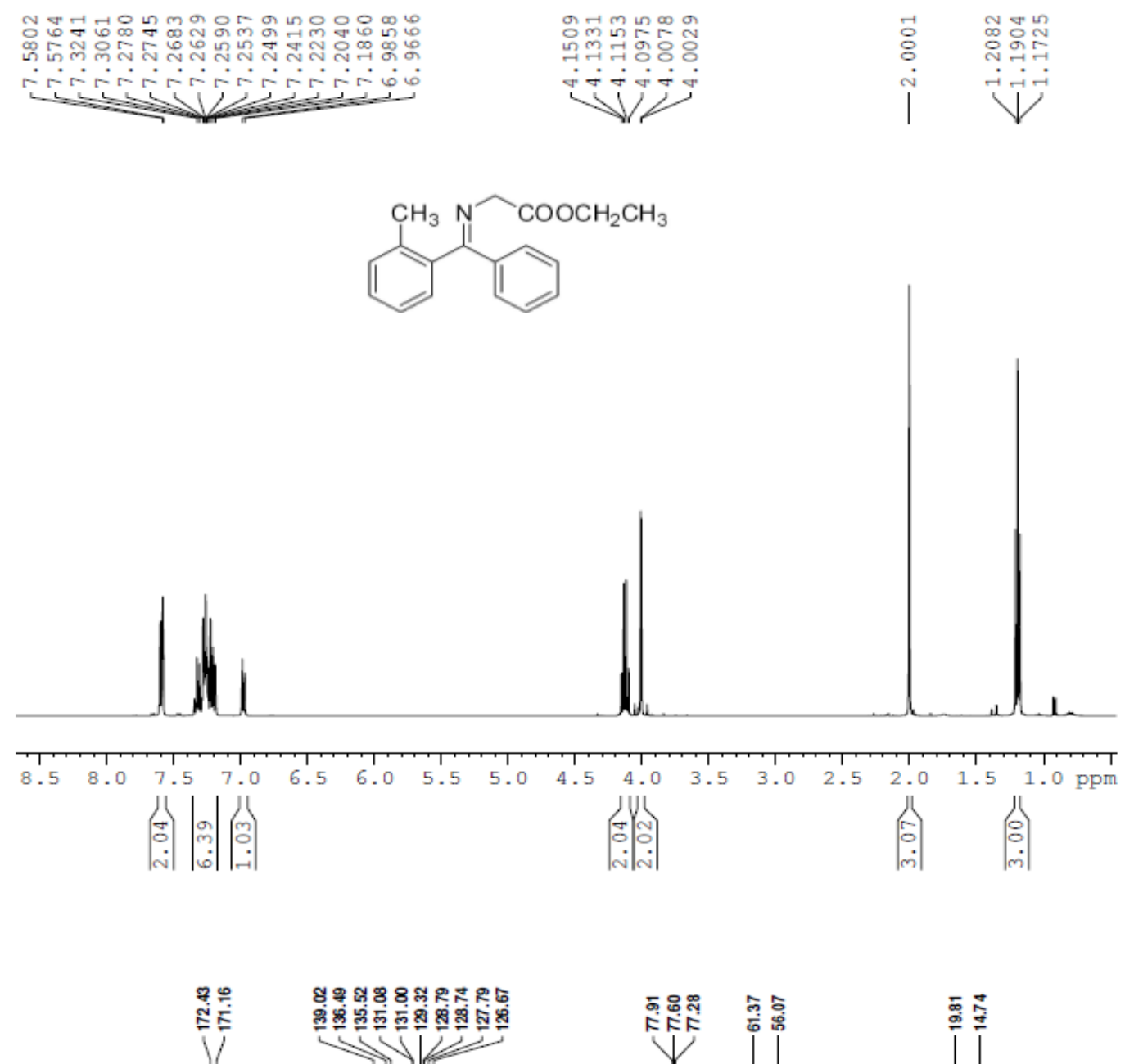

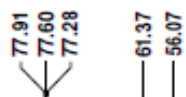

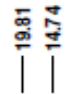
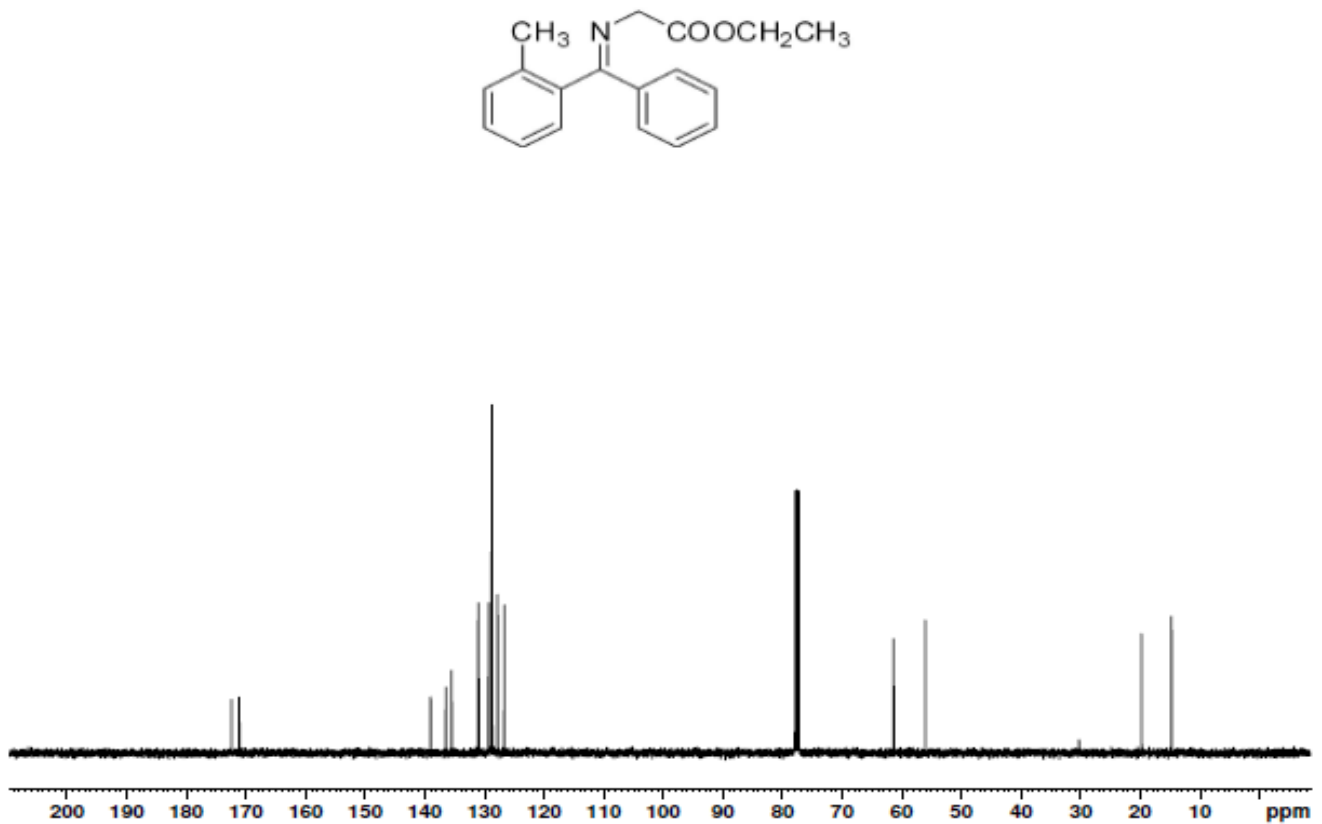
(E)-tert-butyl 2-((phenyl(o-tolyl)methylene)amino)acetate (1v)

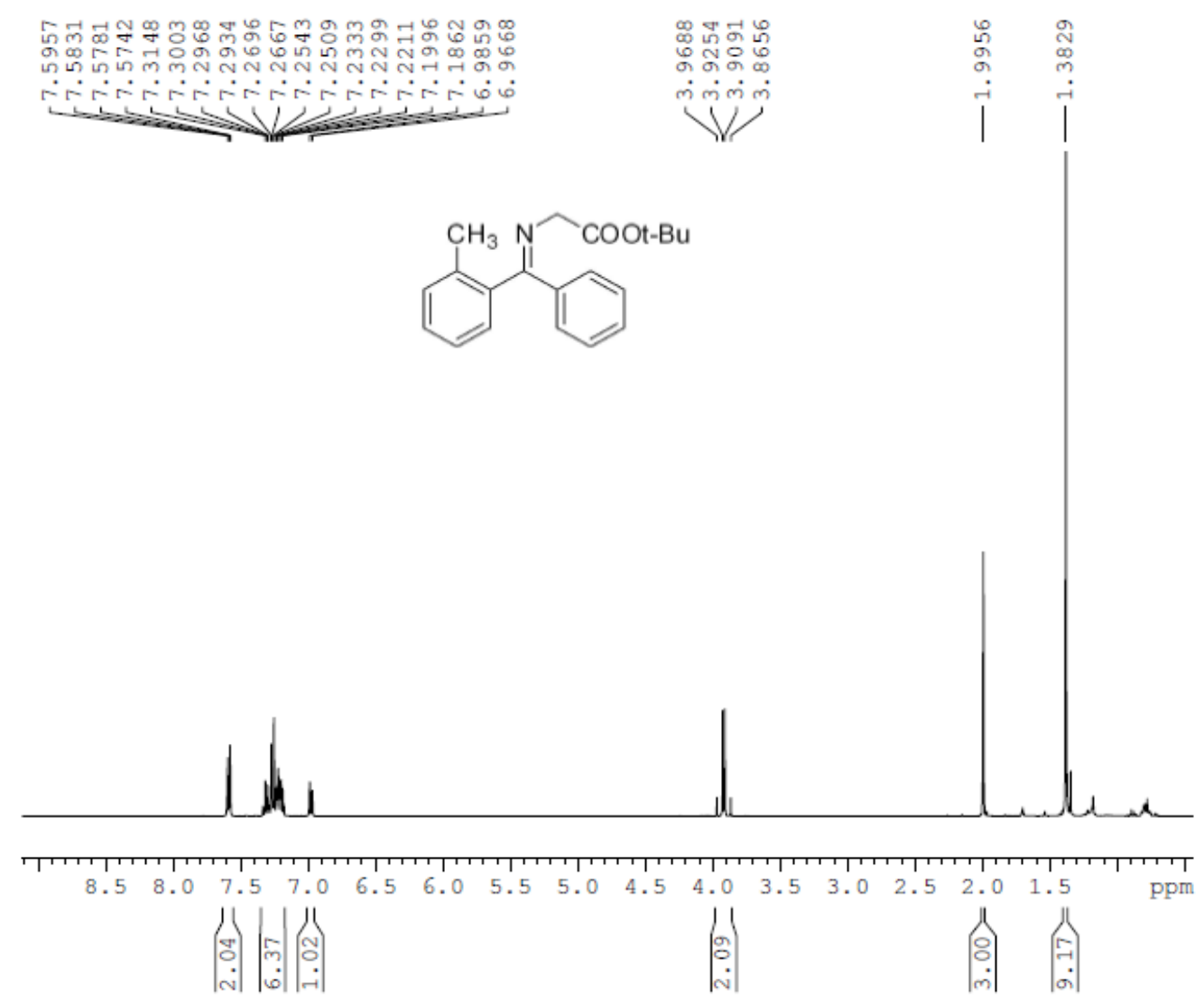

8. 중<smiles>CCOC(=O)CN=C(c1ccccc1)c1ccccc1C</smiles>

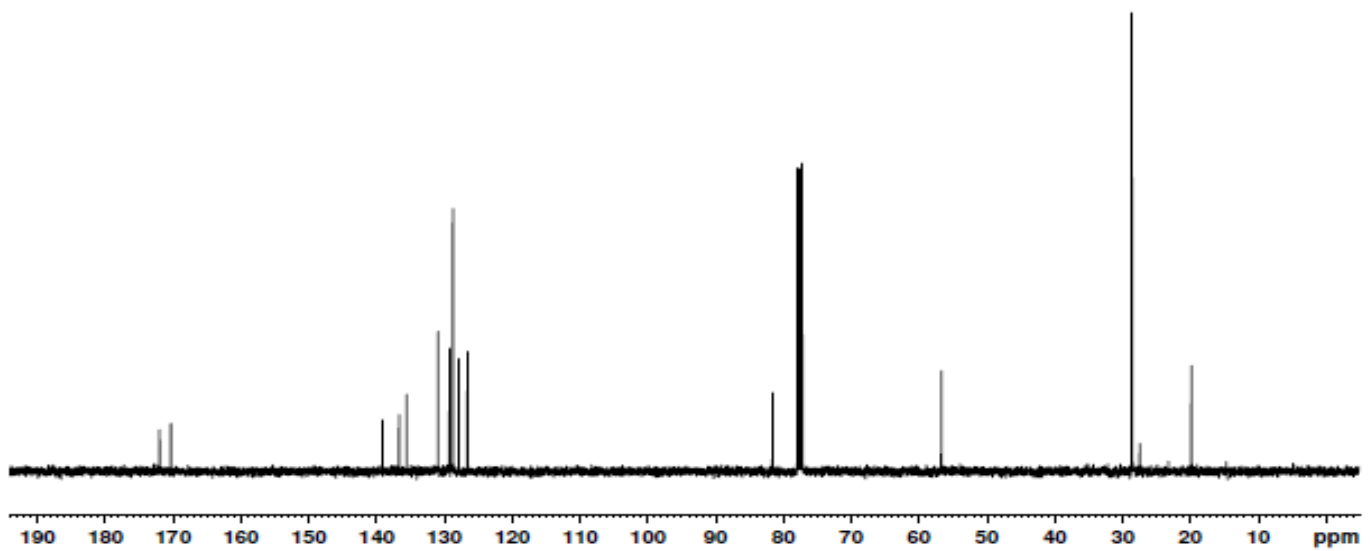


(E)- $N$-(phenyl(o-tolyl)methylene)methanamine (1w)

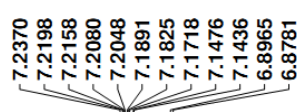

总
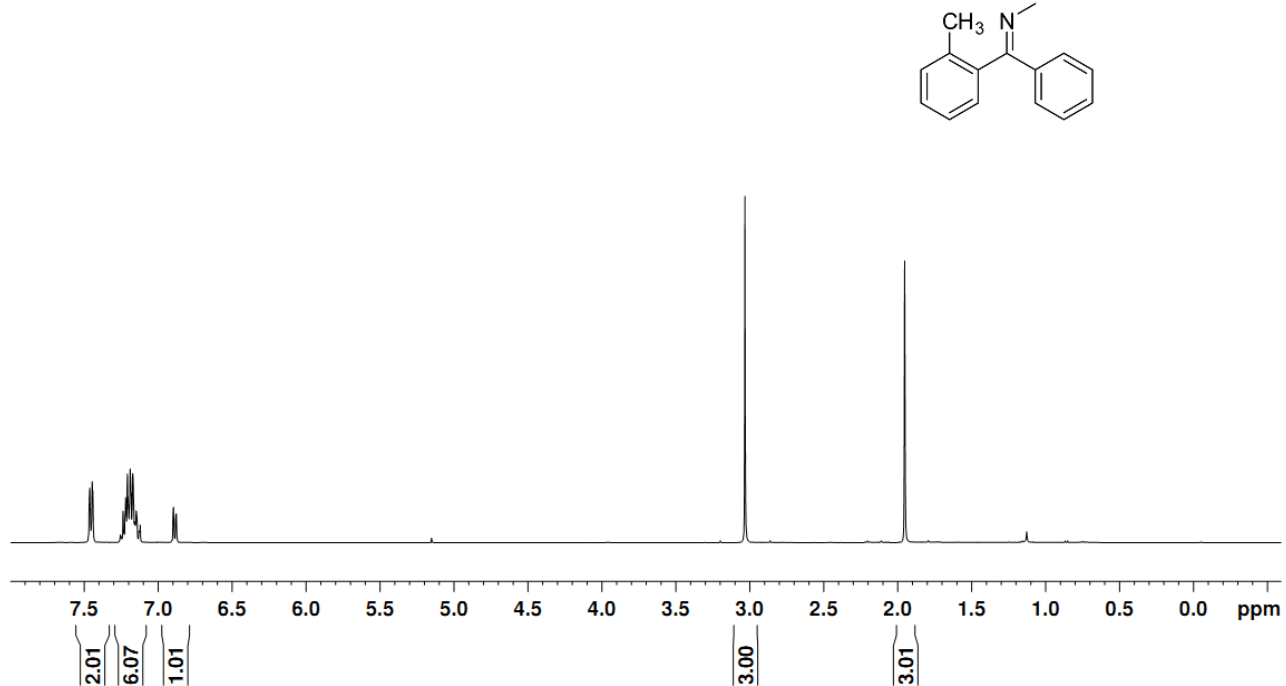

|

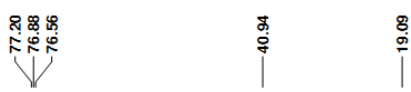
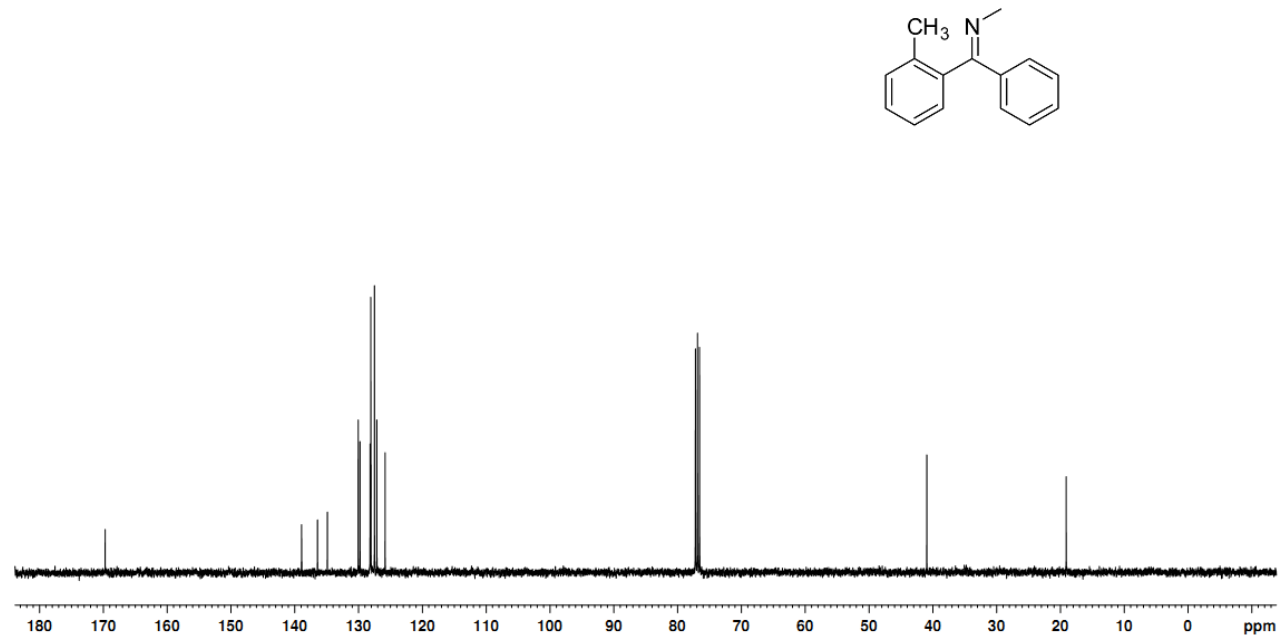
(E)- $N$-(phenyl(o-tolyl)methylene)ethanamine (1x)

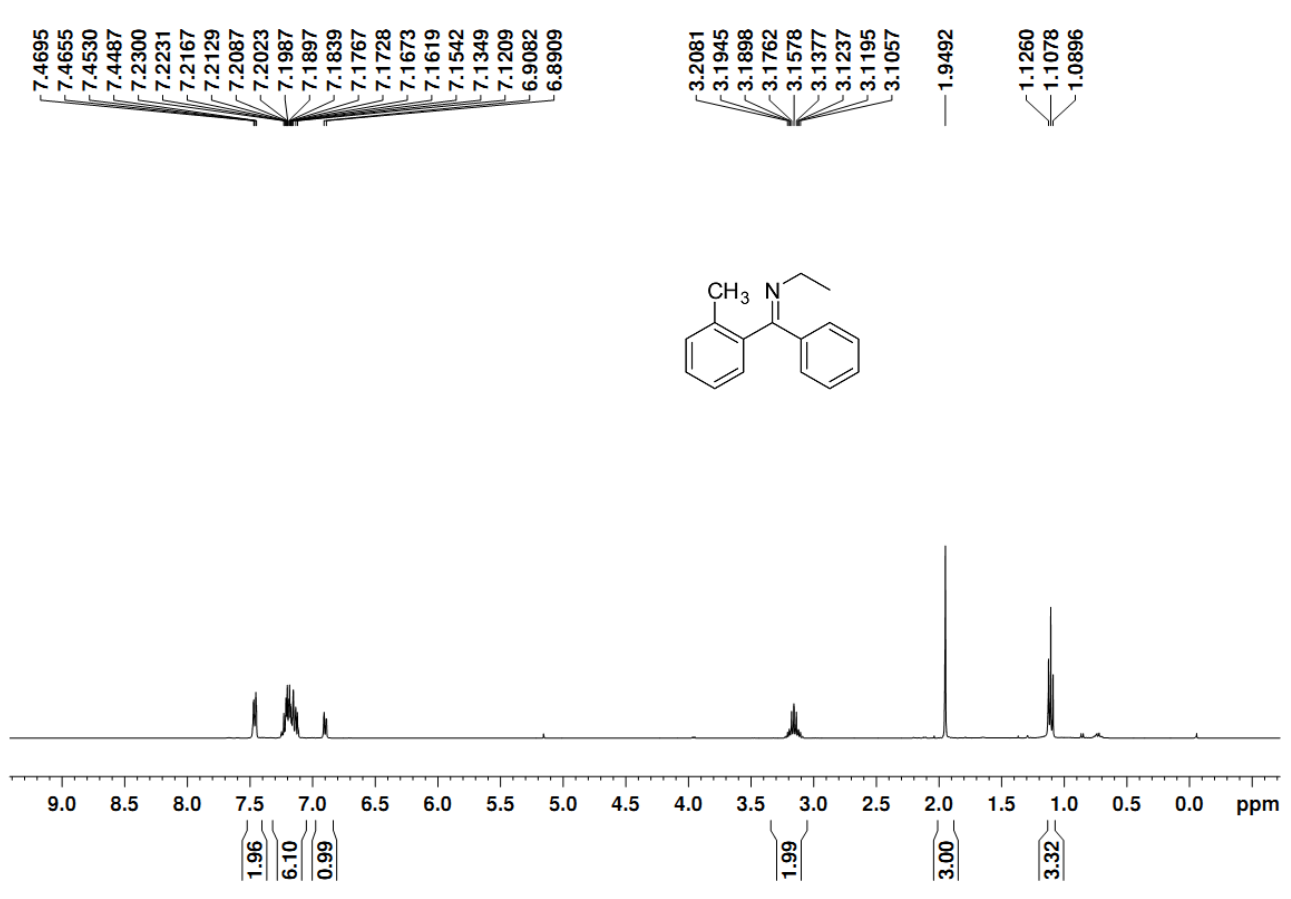

|
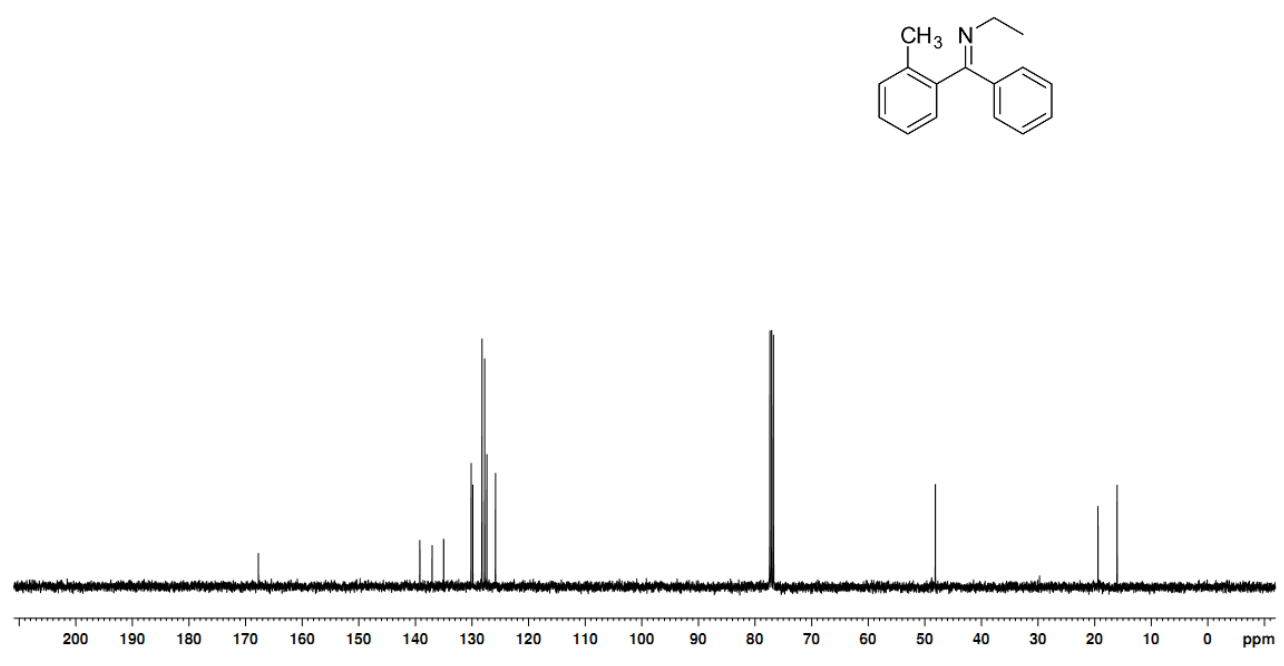
methyl 2-((phenyl(o-tolyl)methyl)amino)acetate (2a)
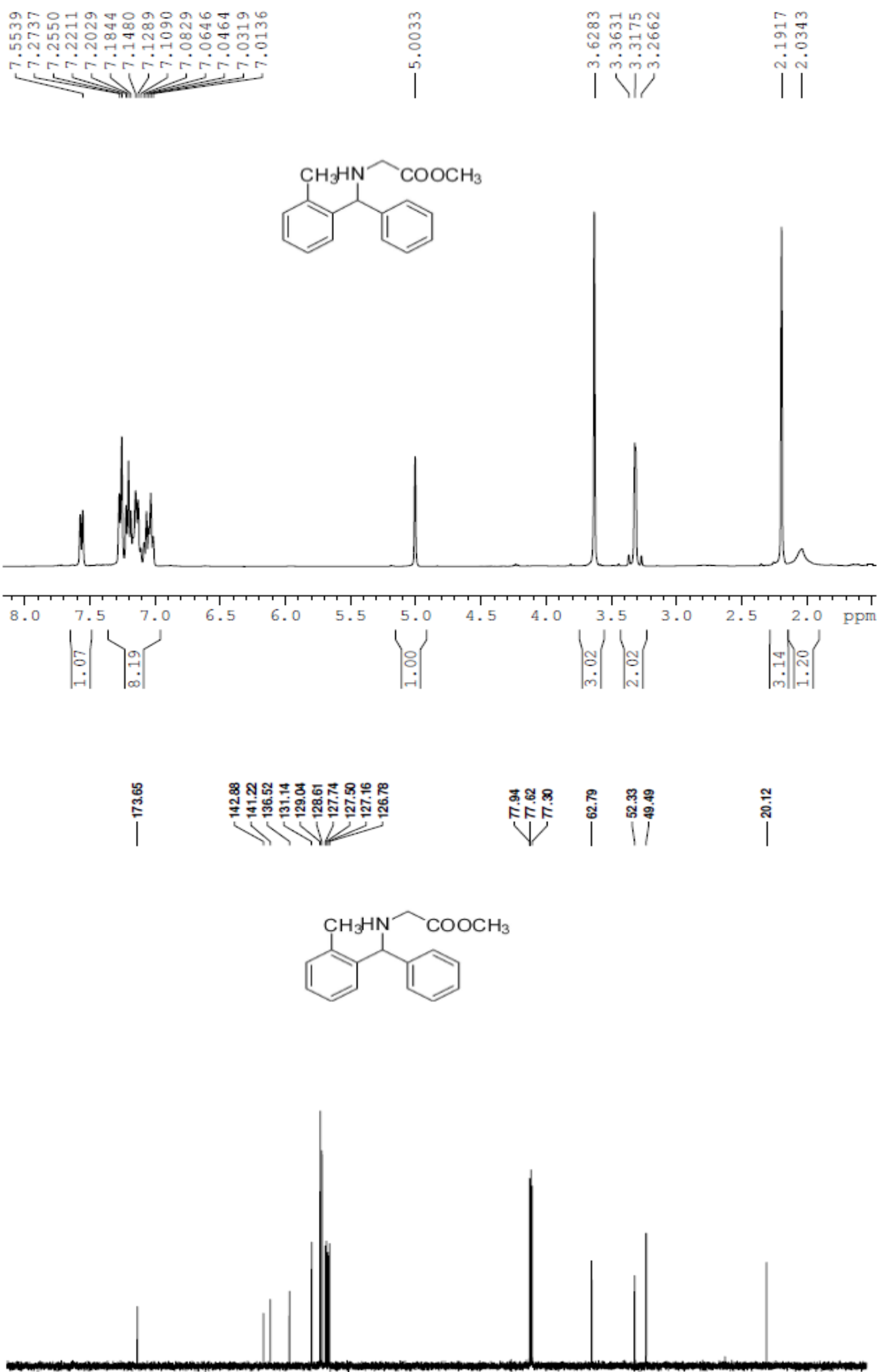

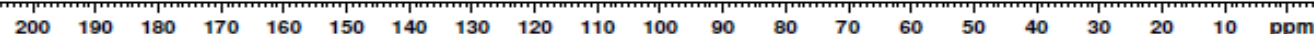


methyl 2-(((3-methoxyphenyl)(o-tolyl)methyl)amino)acetate (2b)
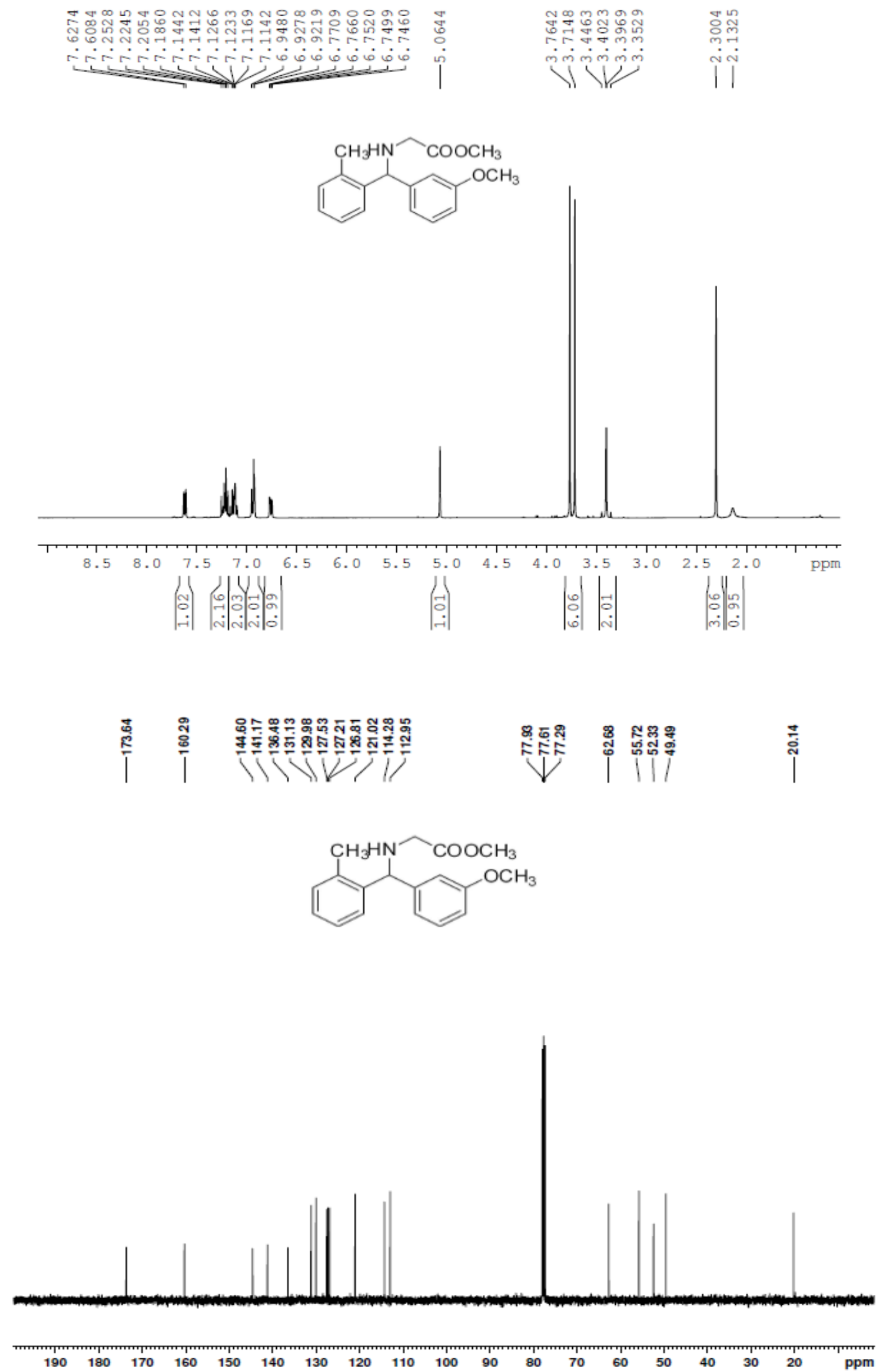
methyl 2-((m-tolyl(o-tolyl)methyl)amino)acetate (2c)

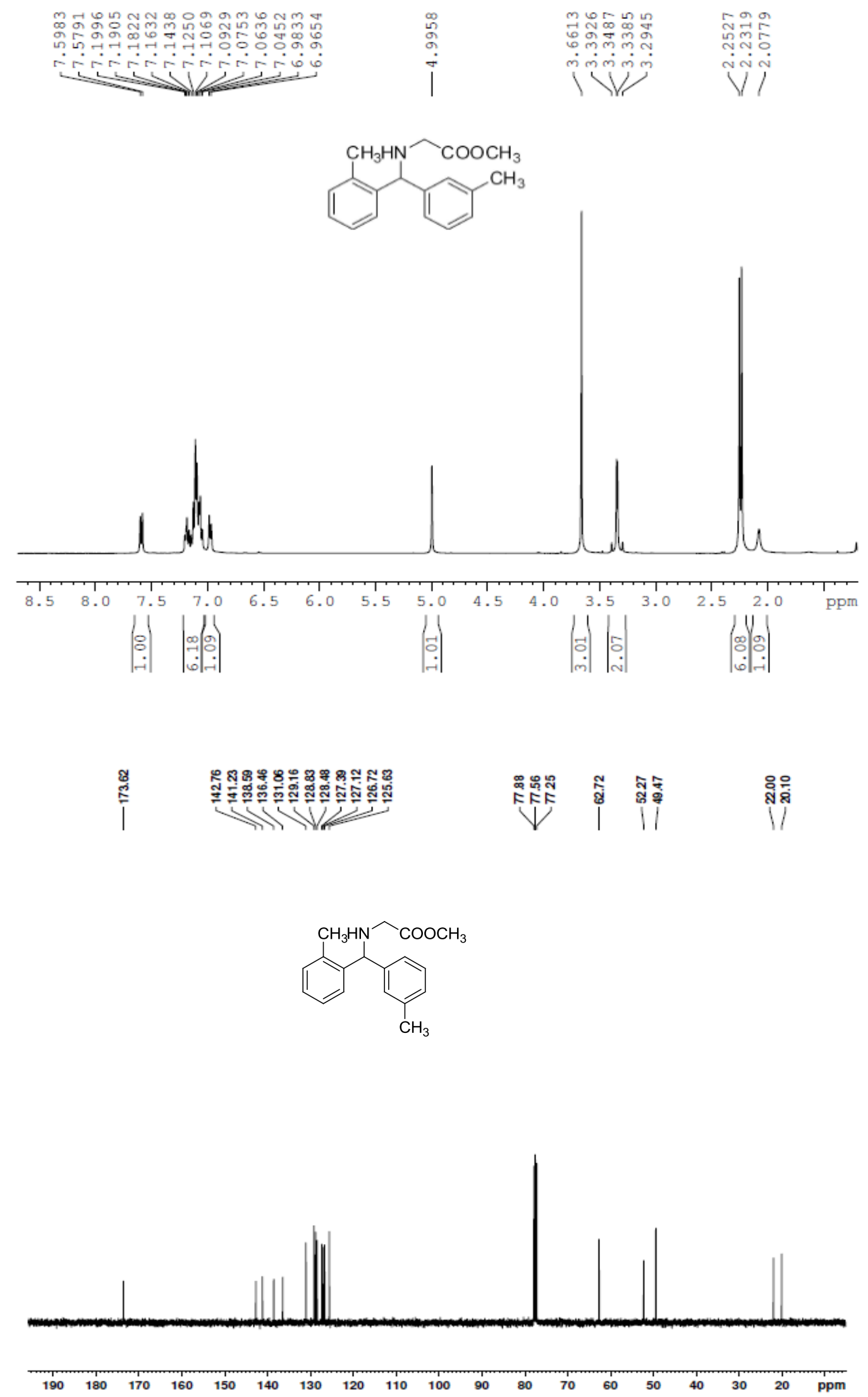


methyl 2-((o-tolyl(p-tolyl)methyl)amino)acetate (2d)

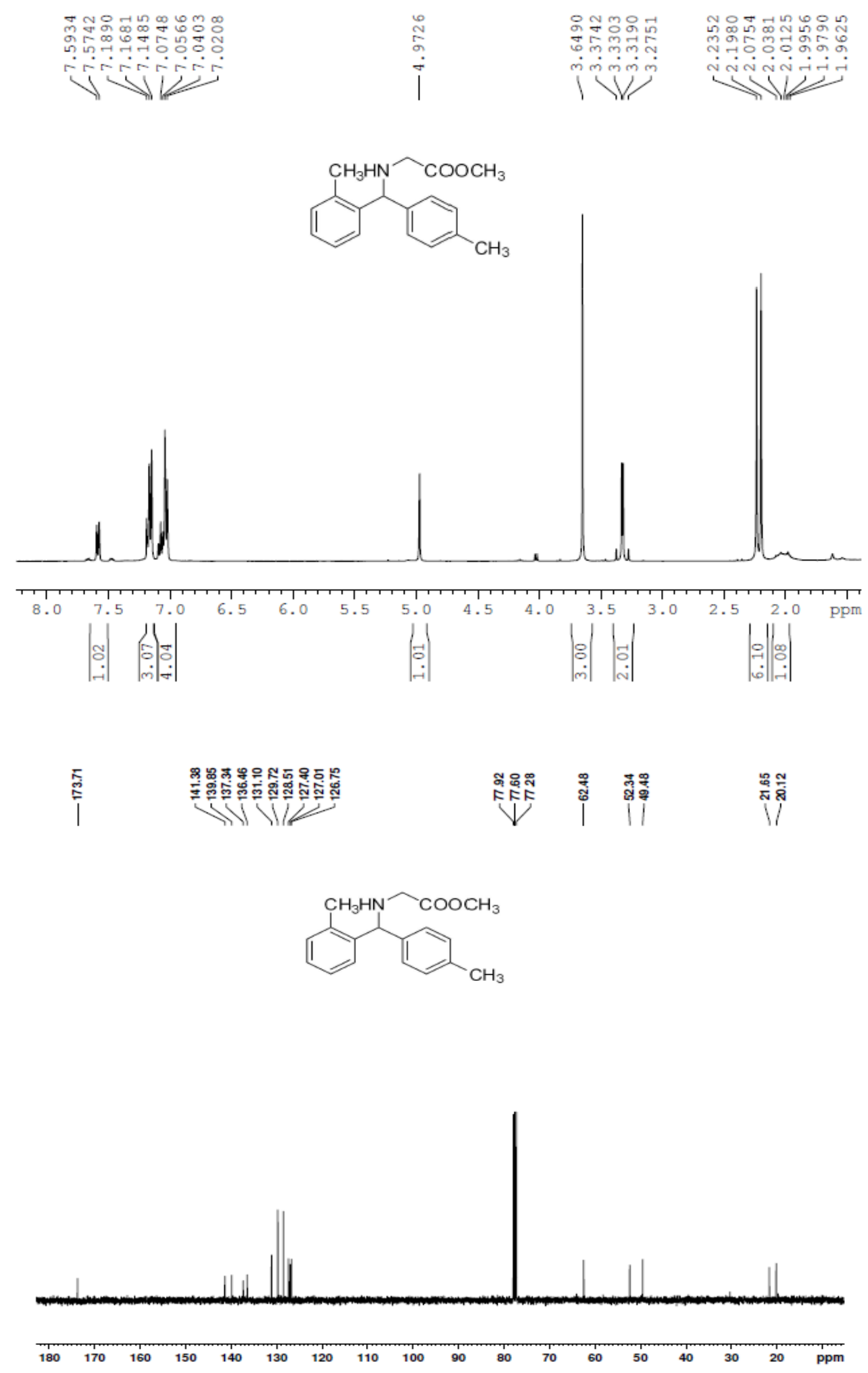


methyl 2-(((4-fluorophenyl)(o-tolyl)methyl)amino)acetate (2e)

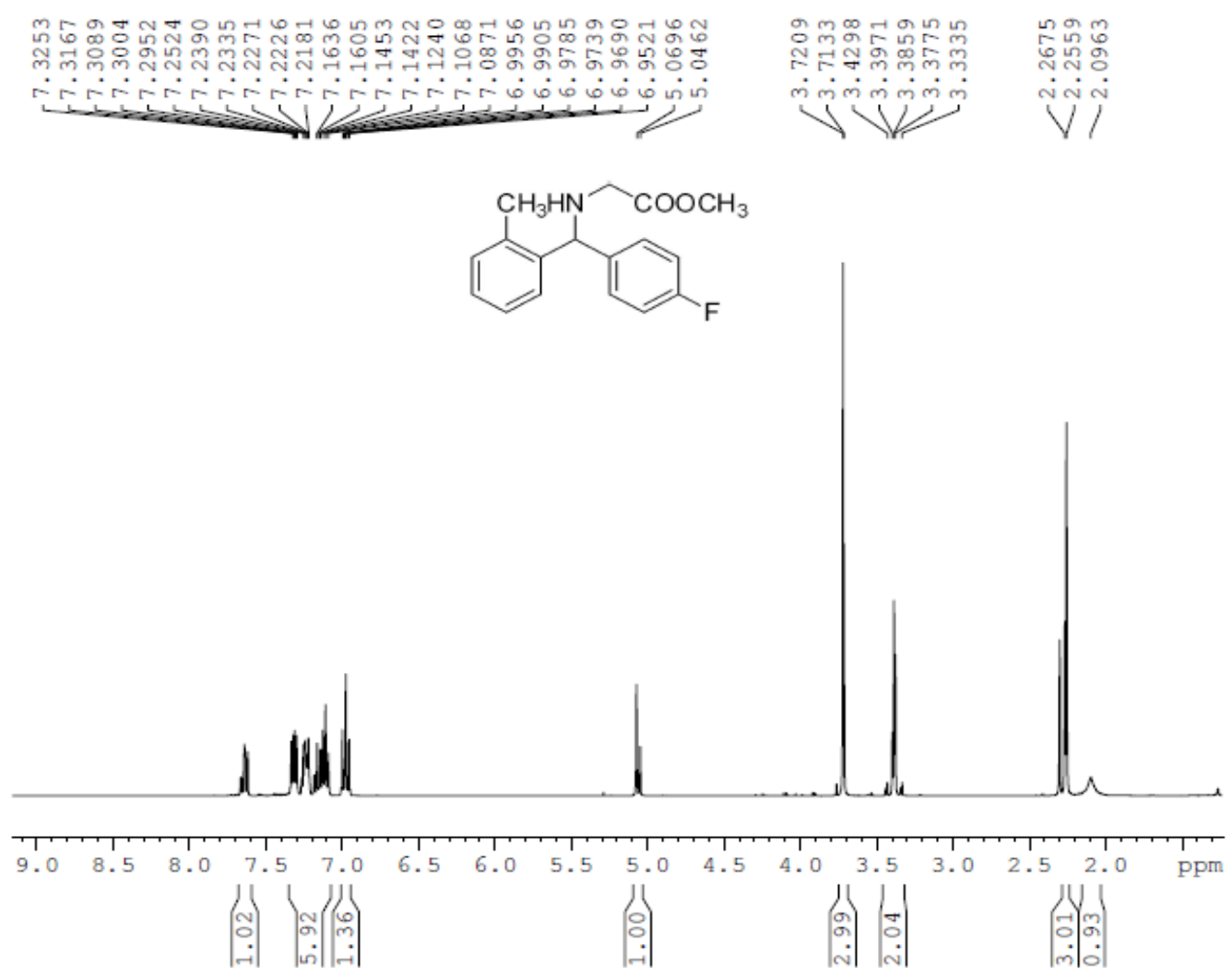

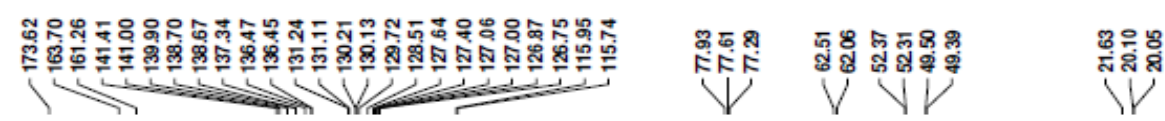
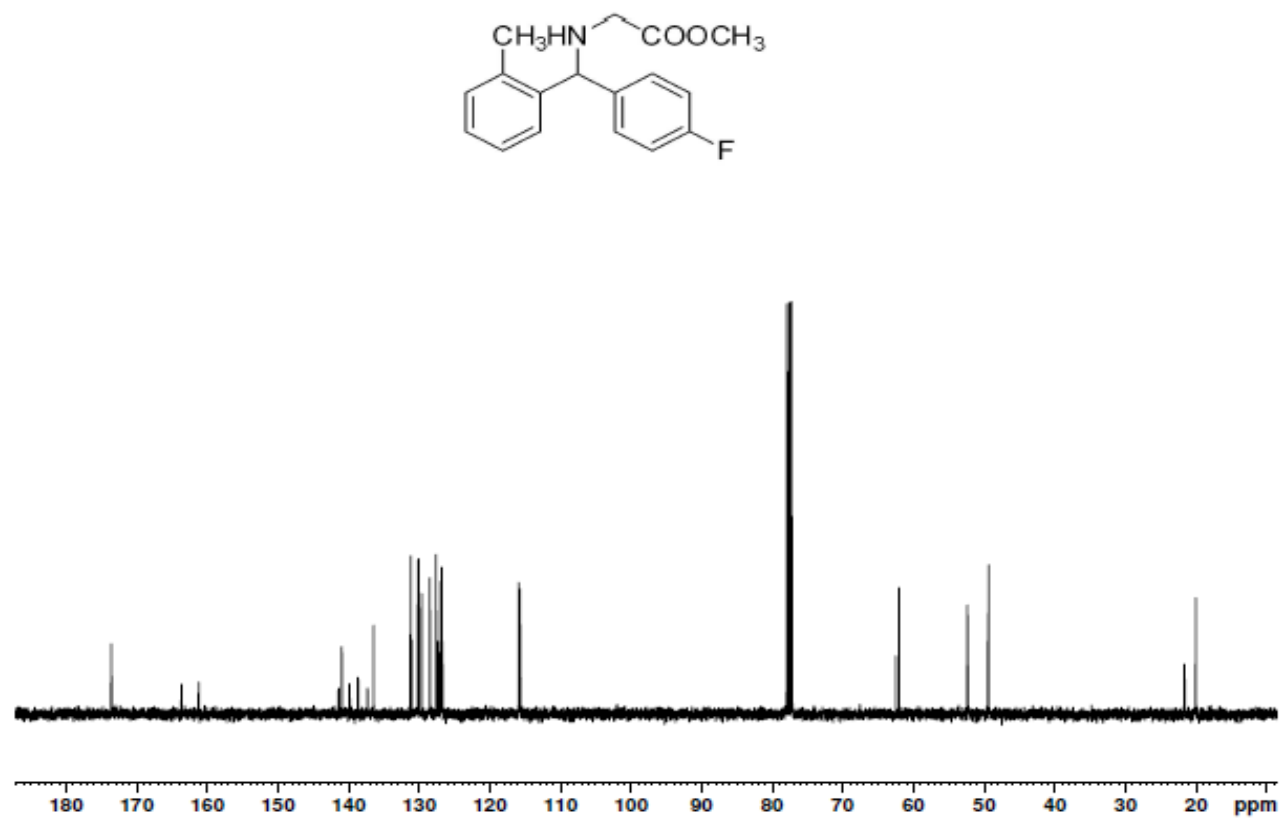
methyl 2-(((2-methoxyphenyl)(phenyl)methyl)amino)acetate (2f)

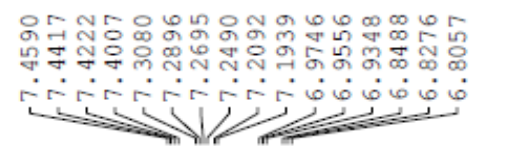<smiles>COC(=O)CNC(c1ccccc1)c1ccccc1OC</smiles>
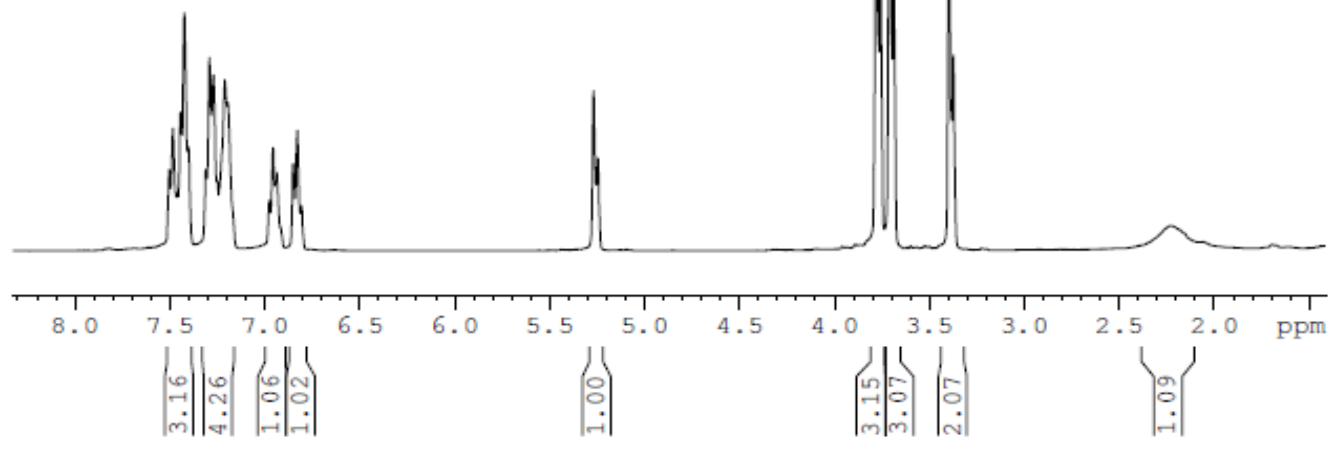

|
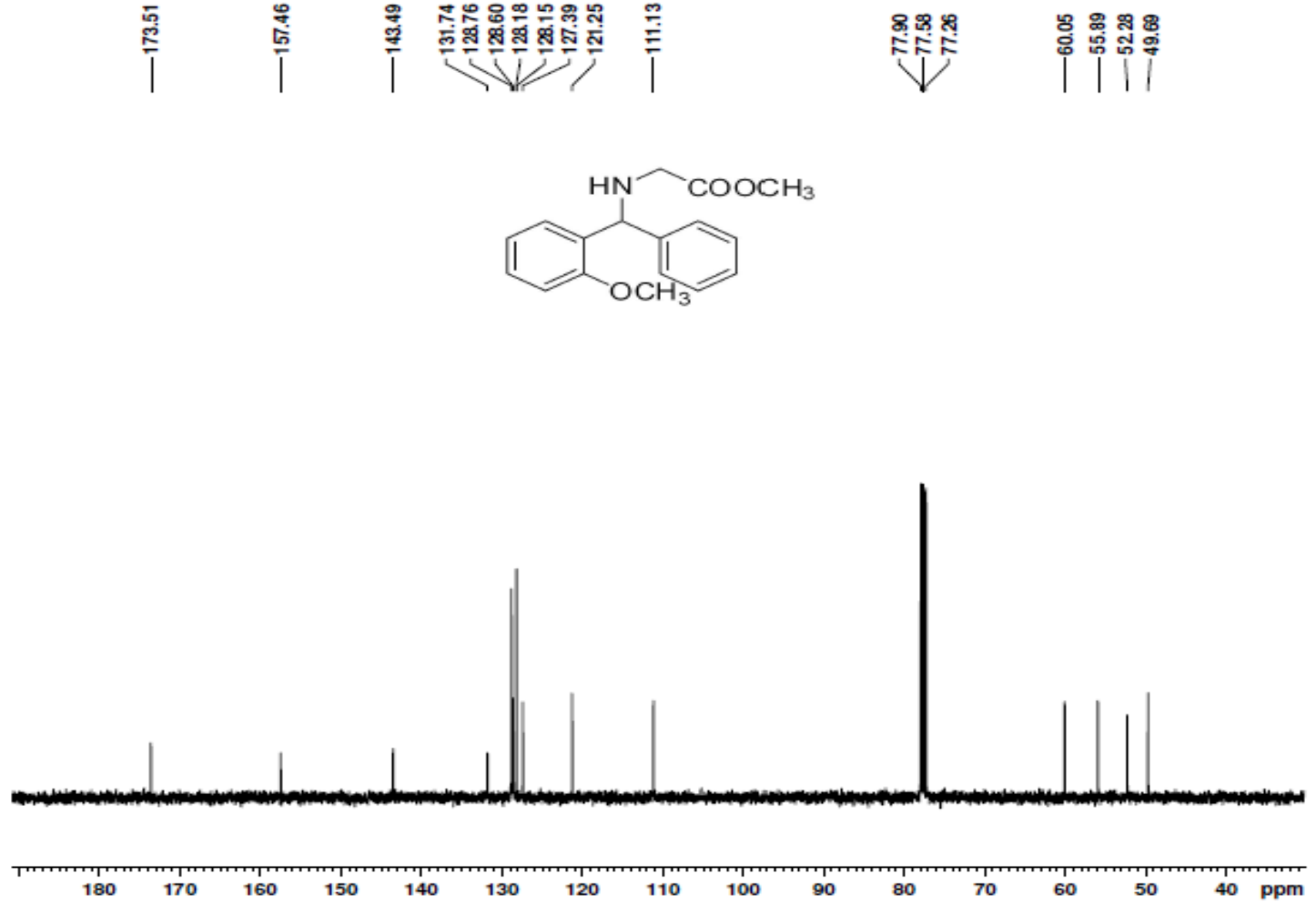
methyl 2-(((2-chlorophenyl)(phenyl)methyl)amino)acetate (2g)
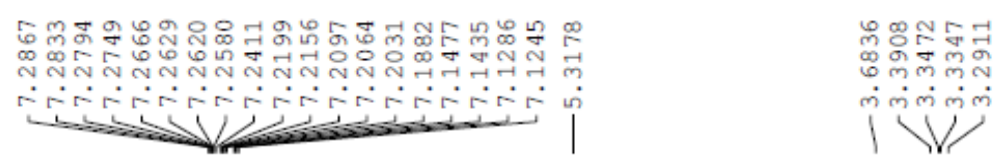

$\underset{1}{\overrightarrow{6}}$
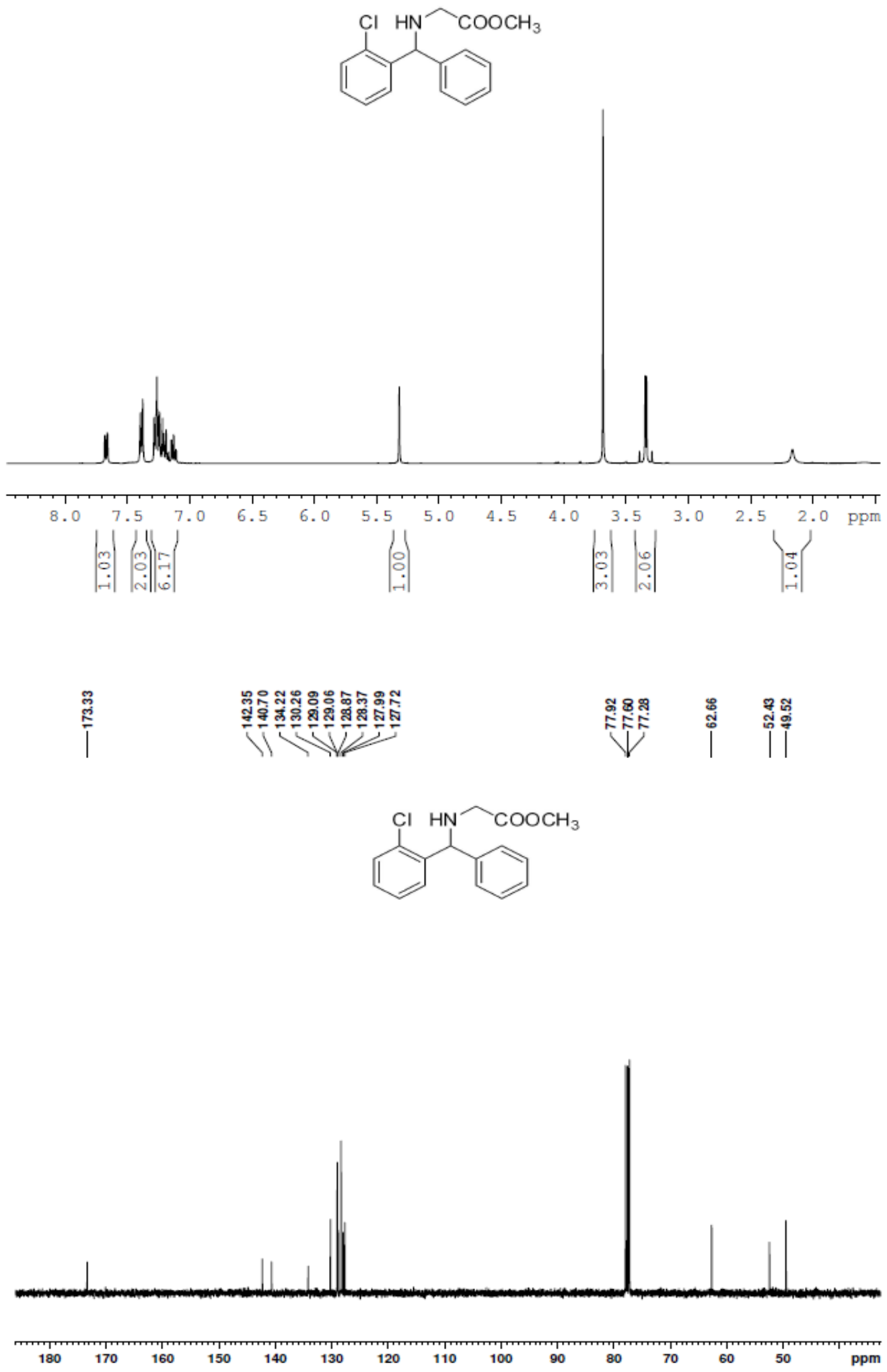

S32 
methyl 2-(((2-chlorophenyl)(3-methoxyphenyl)methyl)amino)acetate (2h)
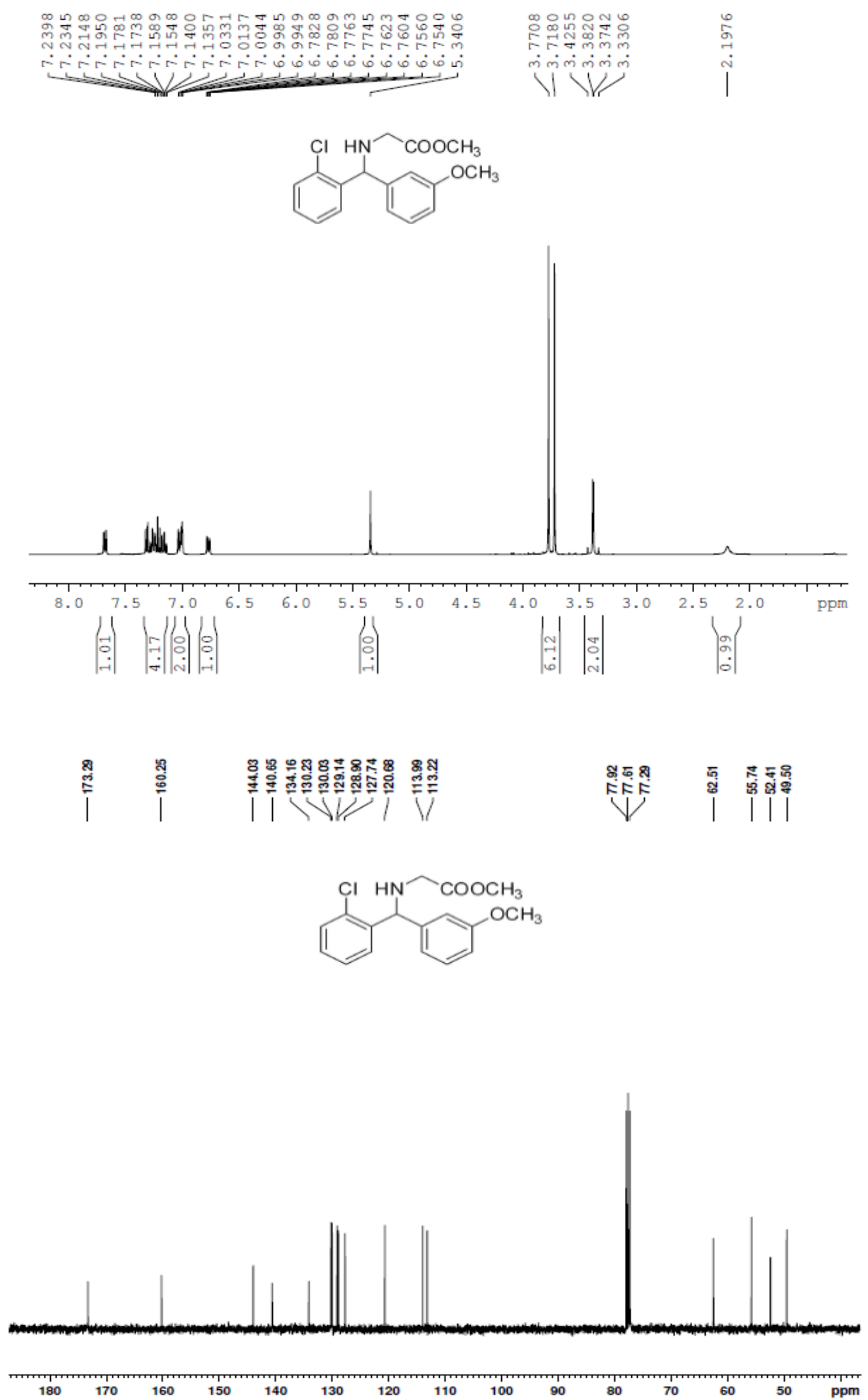
methyl 2-(((2-chlorophenyl)(m-tolyl)methyl)amino)acetate (2i)
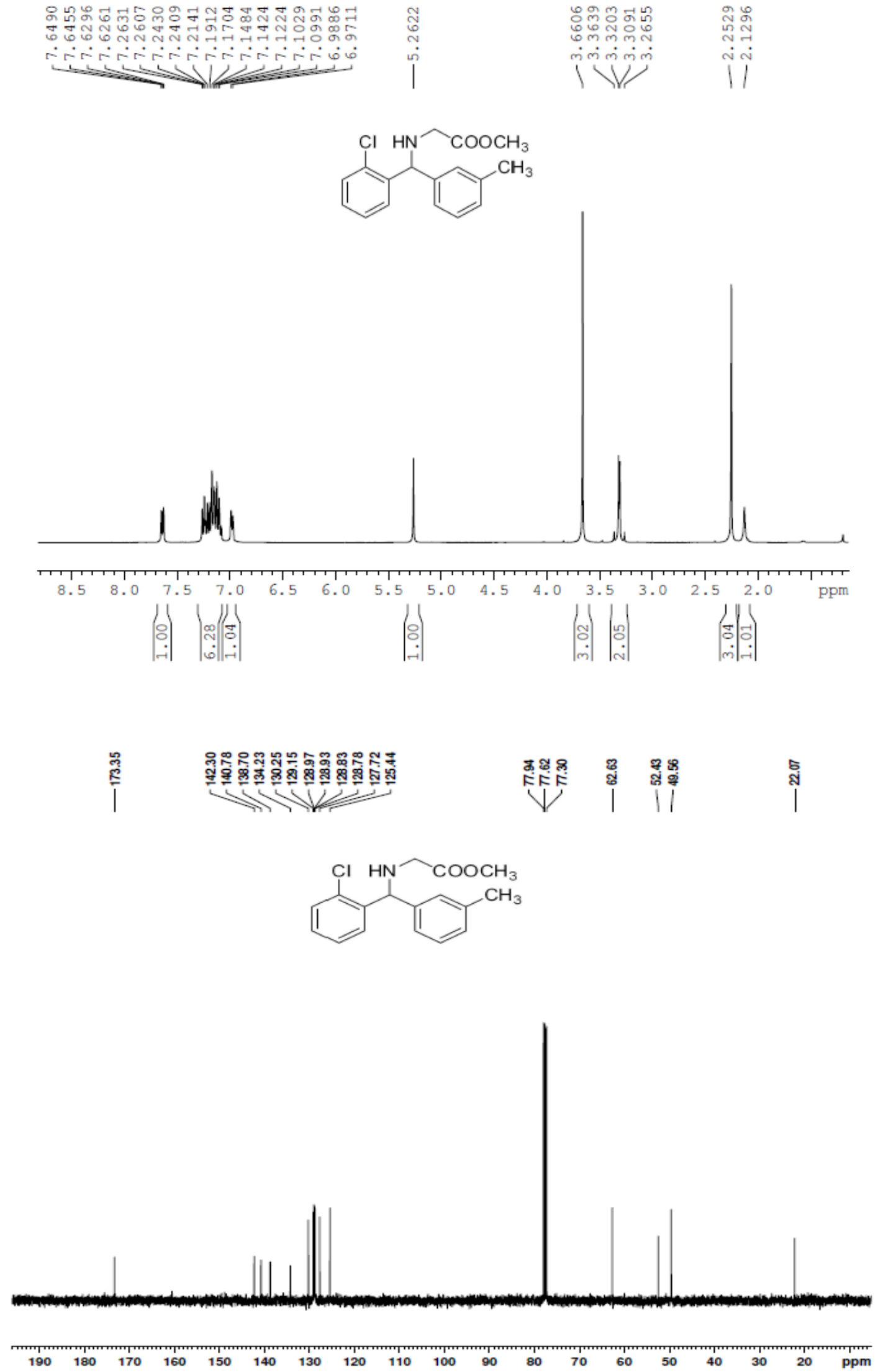
methyl 2-(((2-chlorophenyl)(p-tolyl)methyl)amino)acetate (2j)
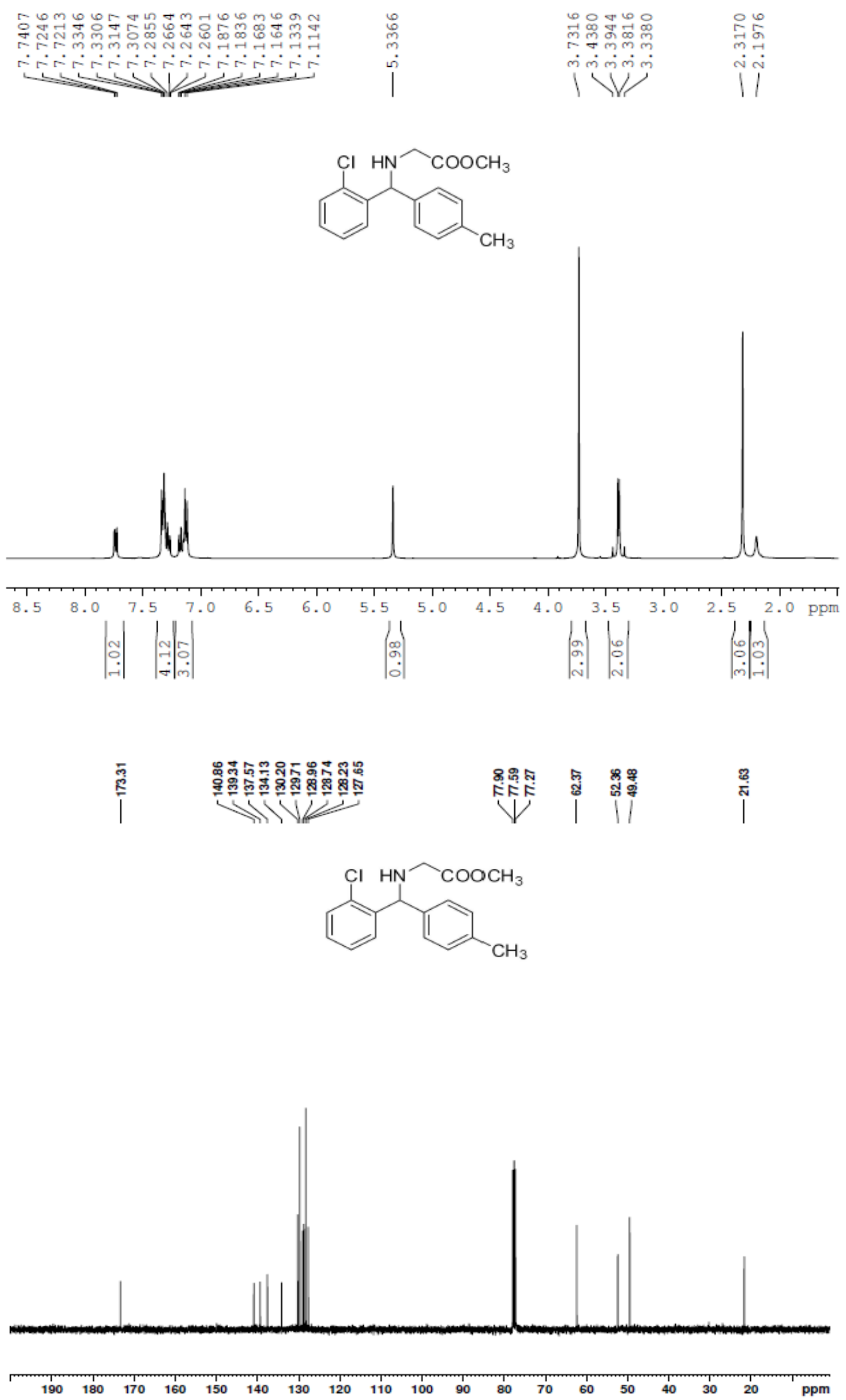
methyl 2-(((2-chlorophenyl)(4-fluorophenyl)methyl)amino)acetate (2k)

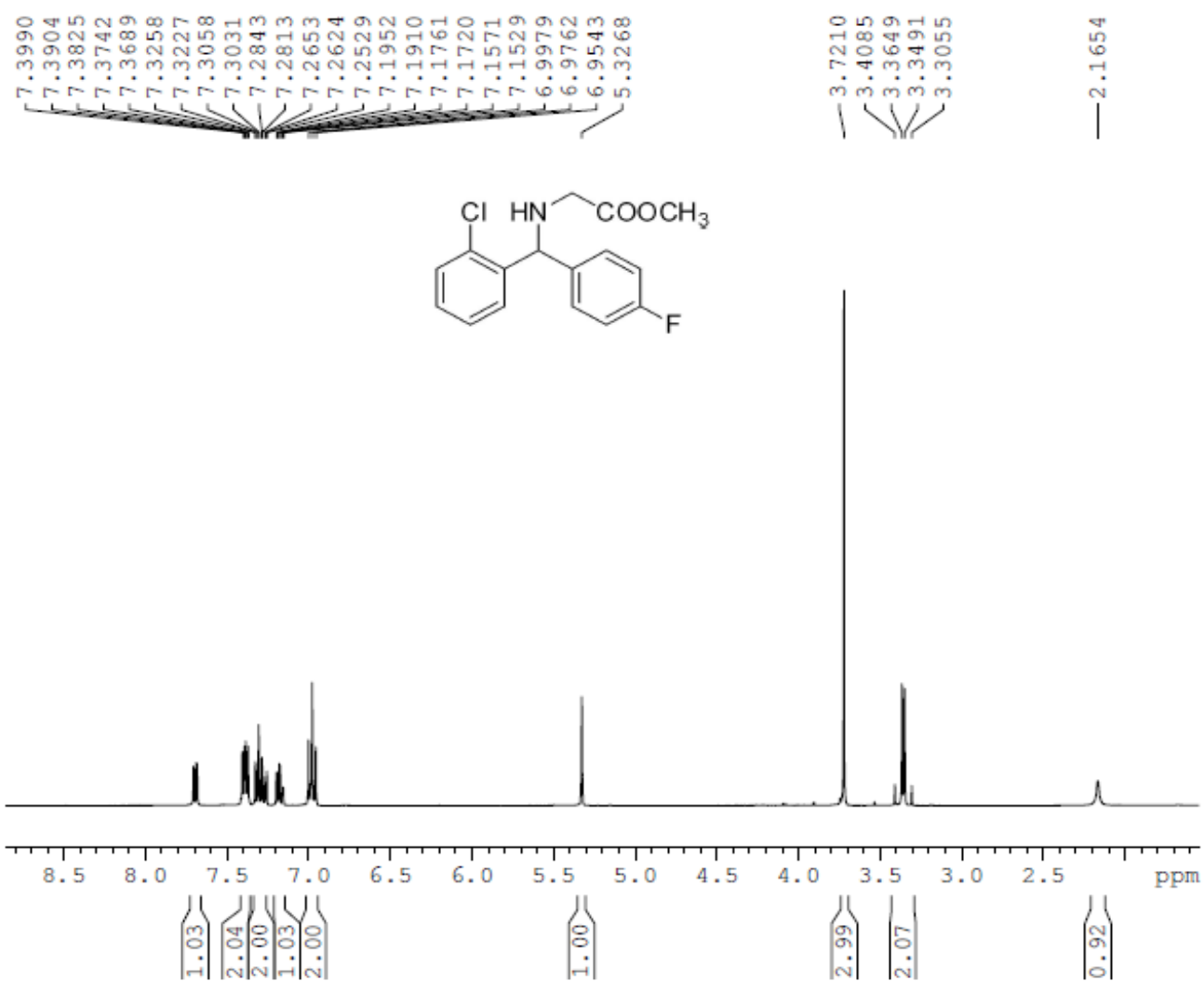

|<smiles>CC(=O)CNC(c1ccc(F)cc1)c1ccccc1Cl</smiles>

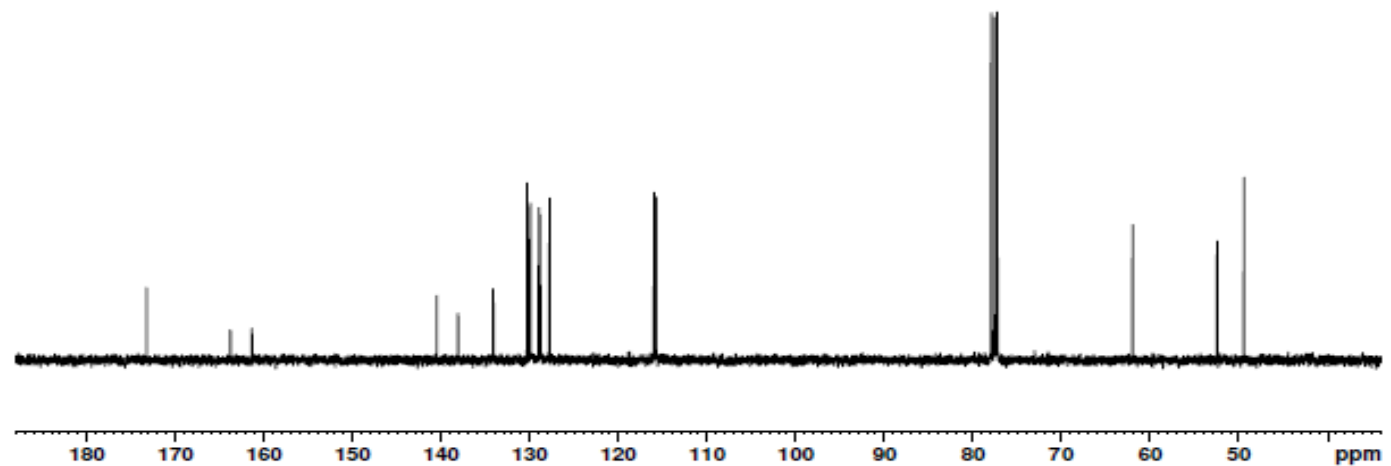


methyl 2-(((2-chlorophenyl)(4-chlorophenyl)methyl)amino)acetate (2I)
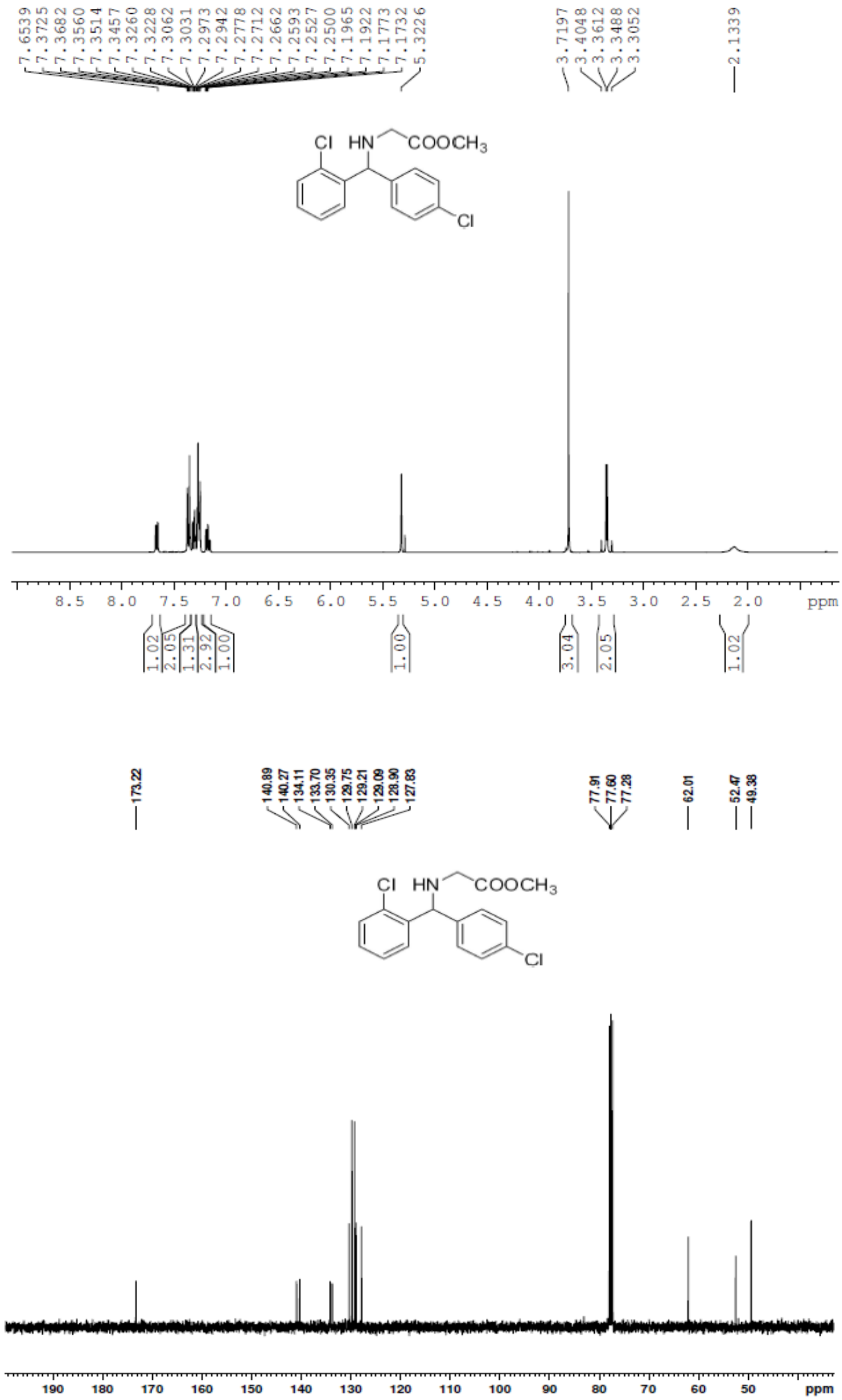
methyl 2-(((2-bromophenyl)(phenyl)methyl)amino)acetate (2m)
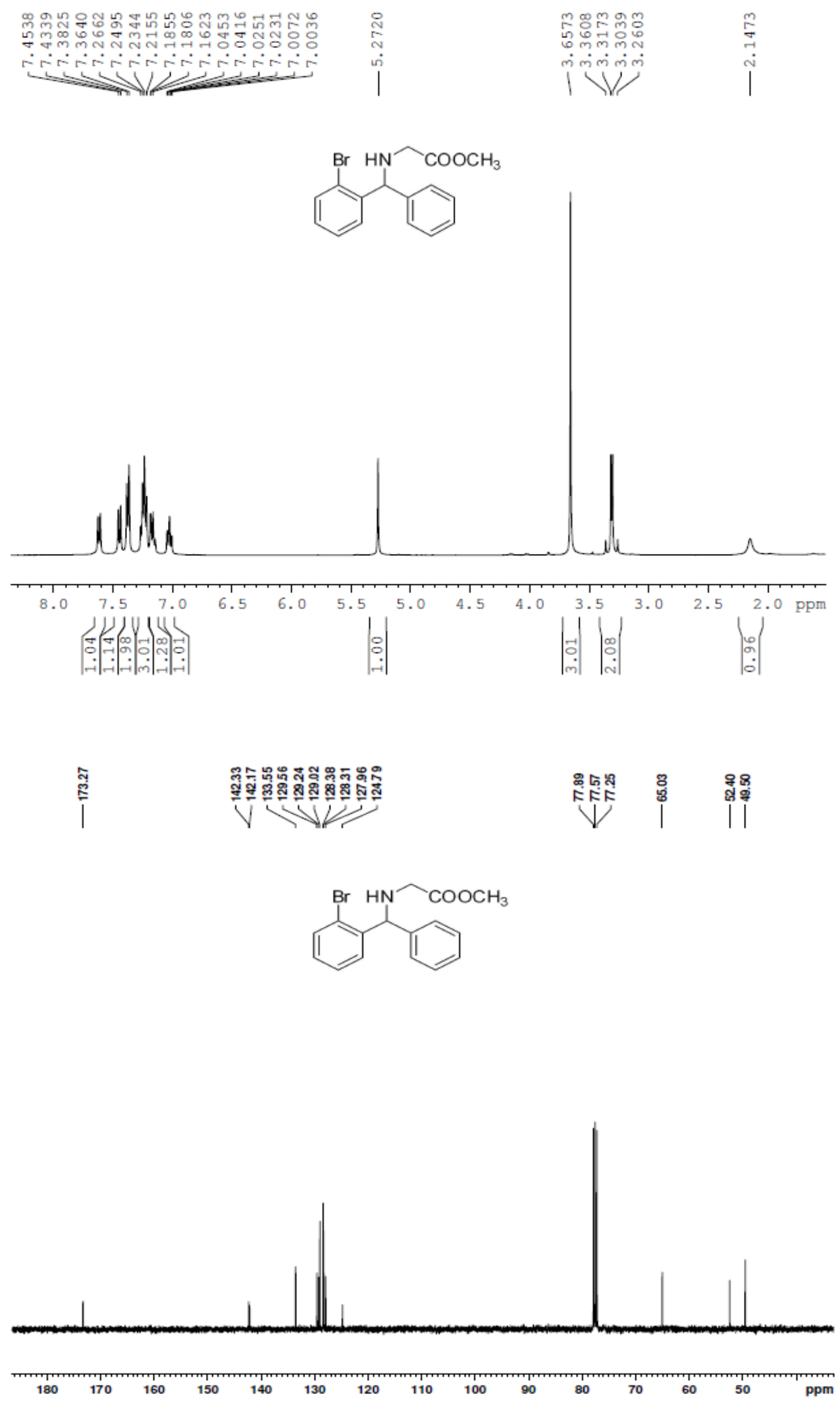
methyl 2-(((2-bromophenyl)(3-fluorophenyl)methyl)amino)acetate (2n)

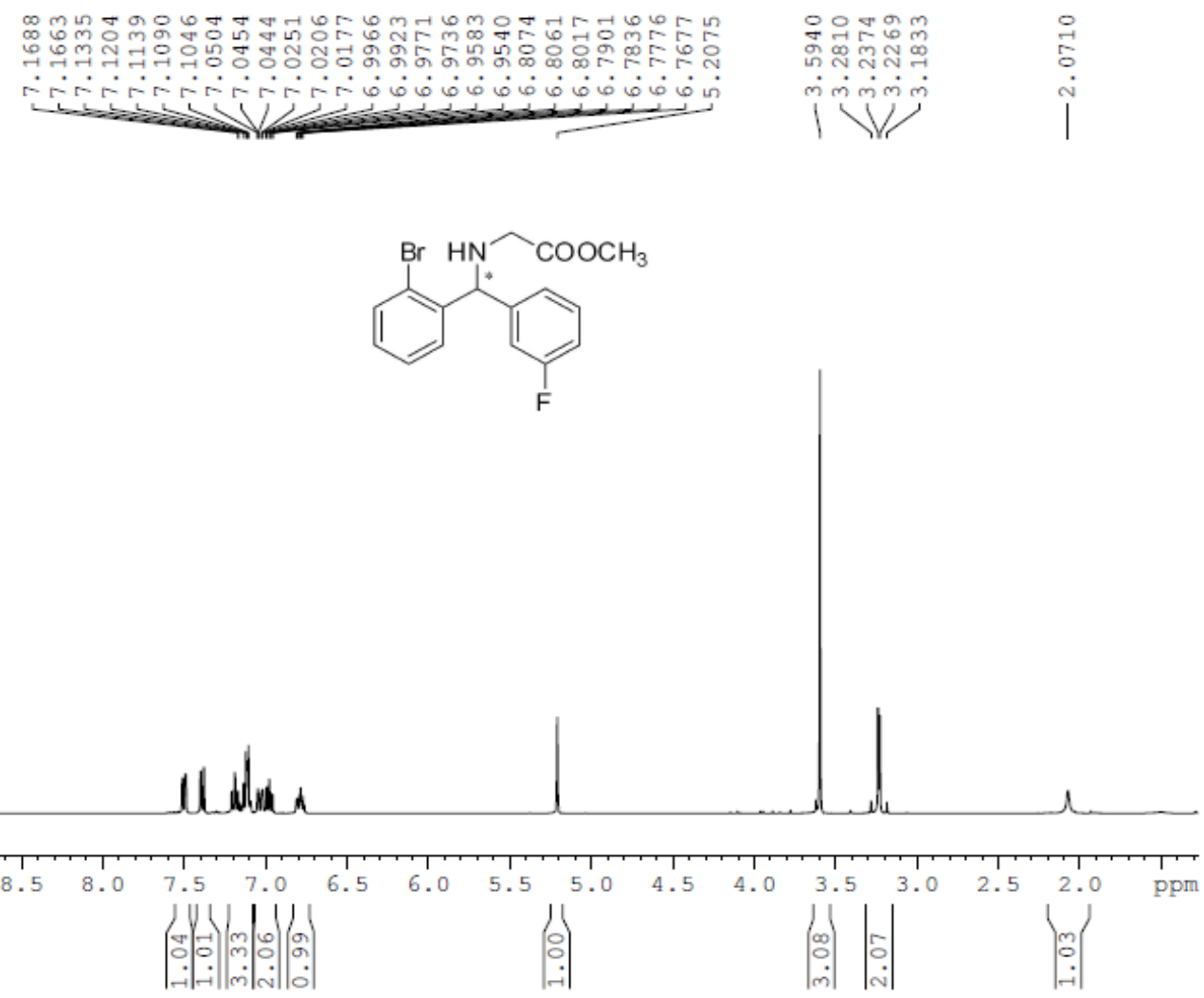

|
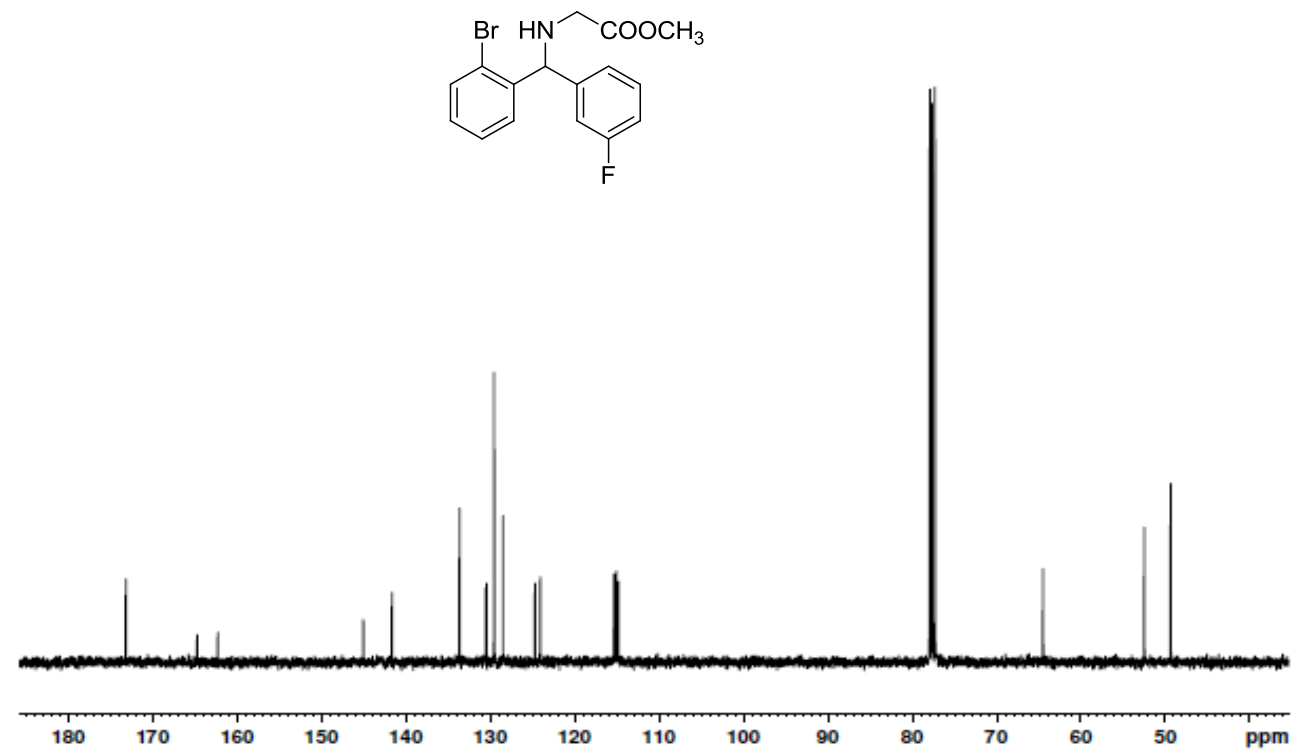
methyl 2-(((2-bromophenyl)(4-fluorophenyl)methyl)amino)acetate (2o)

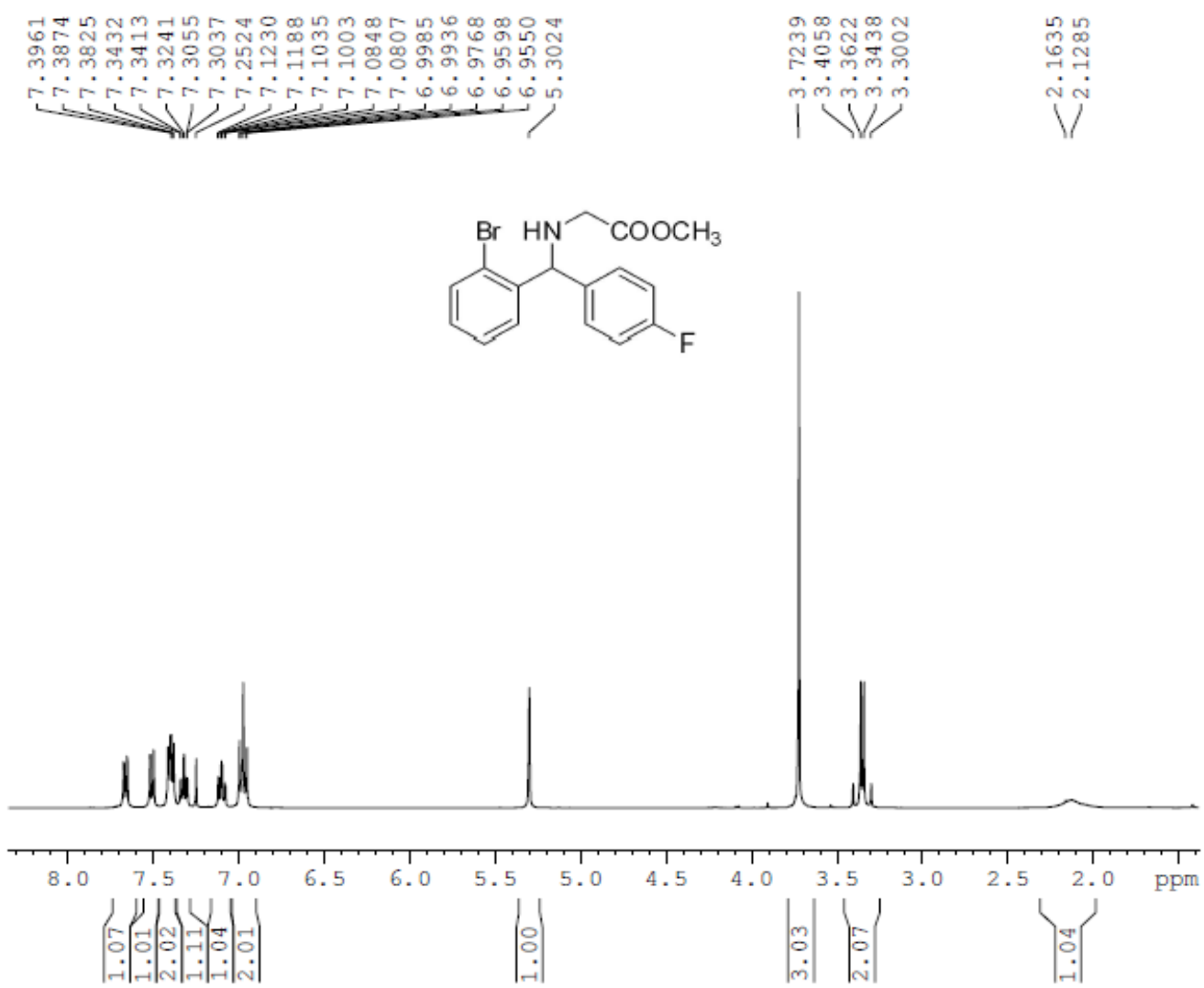

ส<smiles>CC(=O)CNC(c1ccc(F)cc1)c1ccccc1Br</smiles>

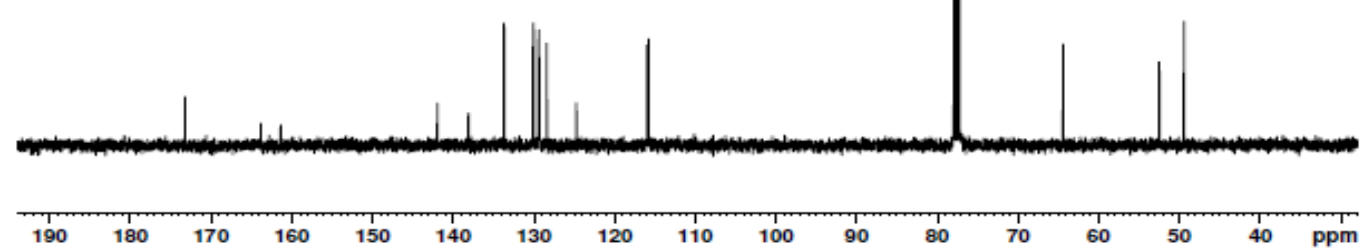


methyl 2-(((2-bromophenyl)(4-chlorophenyl)methyl)amino)acetate (2p)
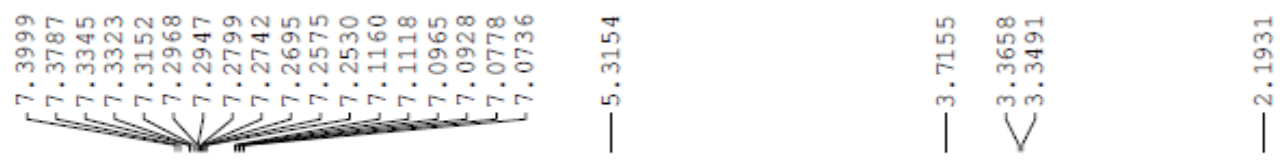<smiles>CC(=O)CNC(c1ccc(Cl)cc1)c1ccccc1Br</smiles>

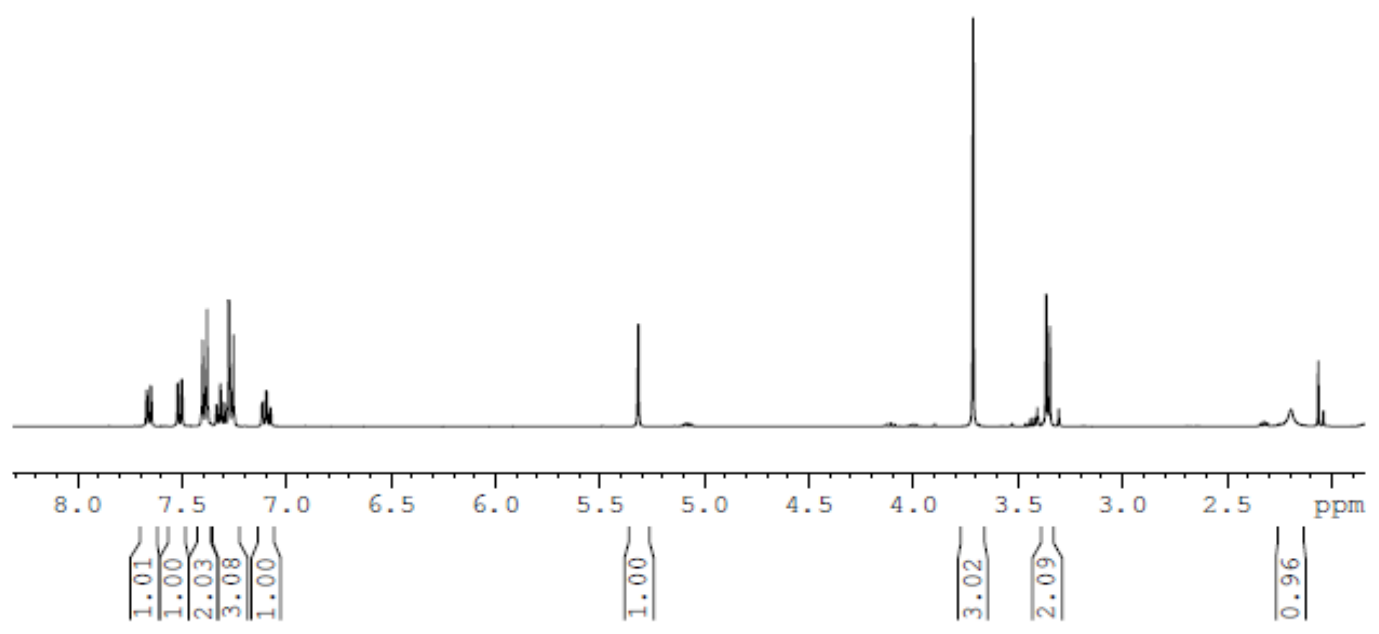

$\stackrel{\frac{m}{m}}{\frac{m}{6}}$

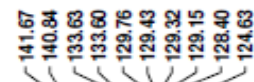

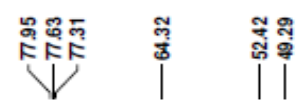<smiles>CC(=O)CNC(c1ccc(Cl)cc1)c1ccccc1Br</smiles>

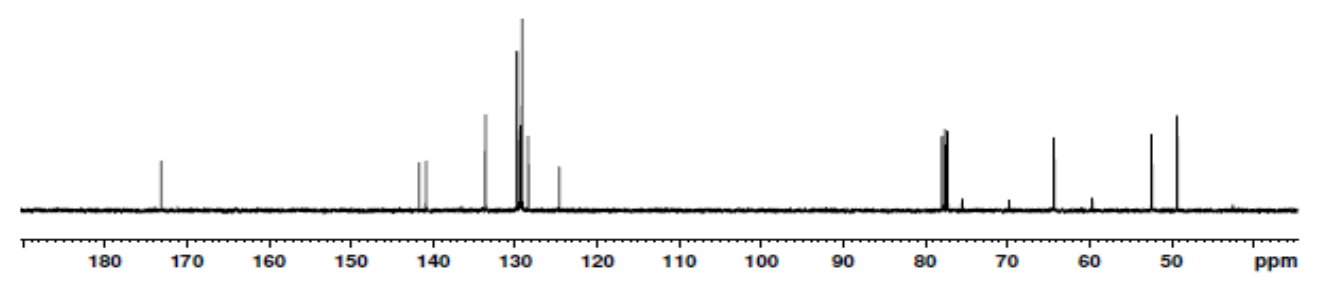


methyl 2-(((2-fluorophenyl)(phenyl)methyl)amino)acetate (2q)
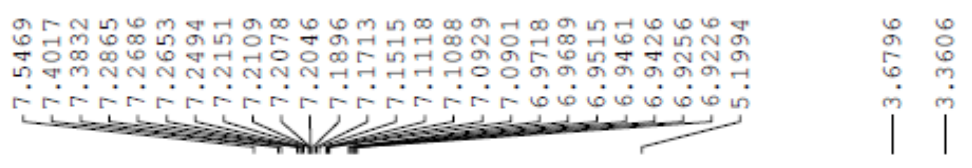

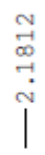
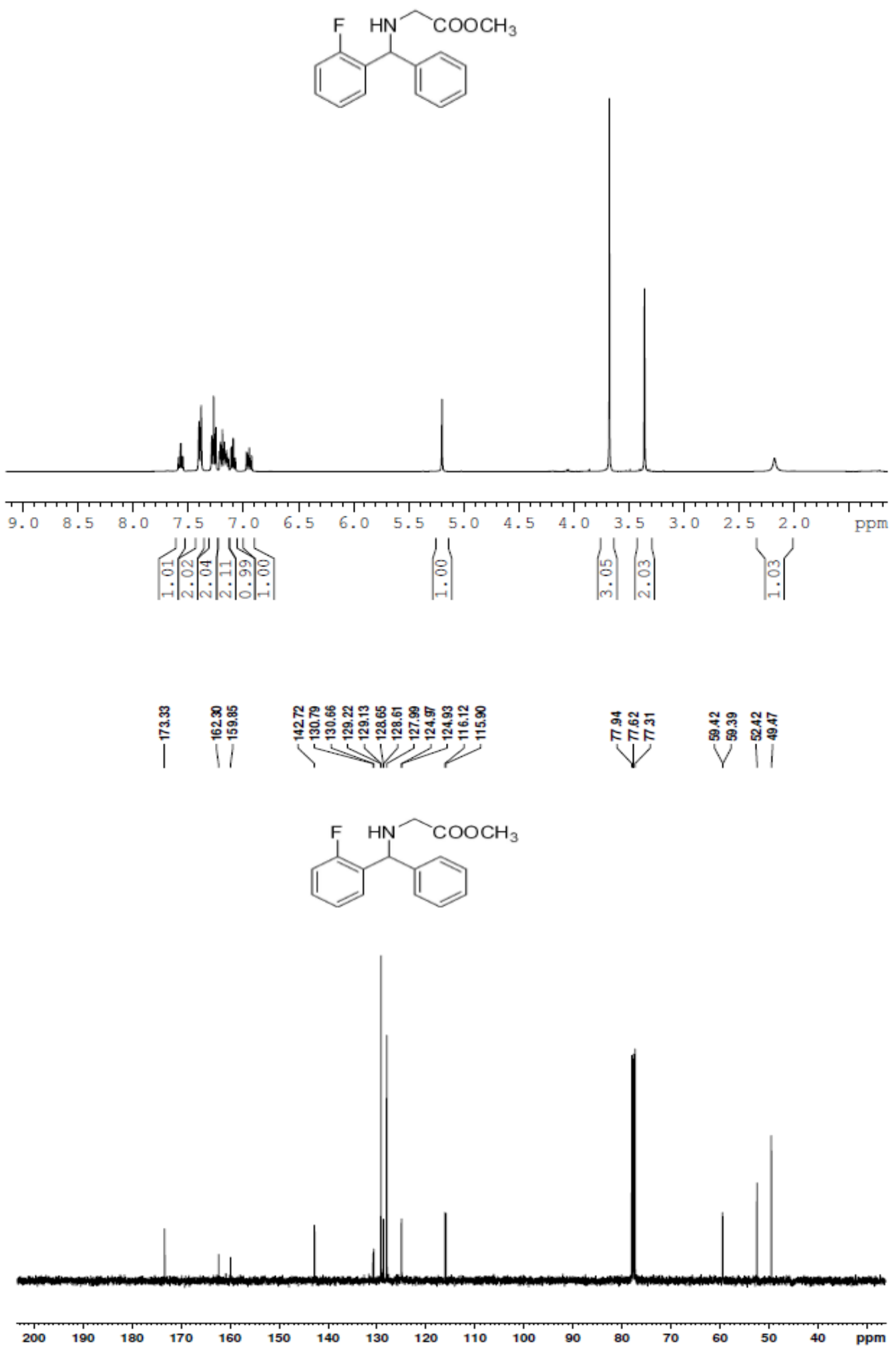

S42 
methyl 2-(((2-fluorophenyl)(m-tolyl)methyl)amino)acetate (2r)

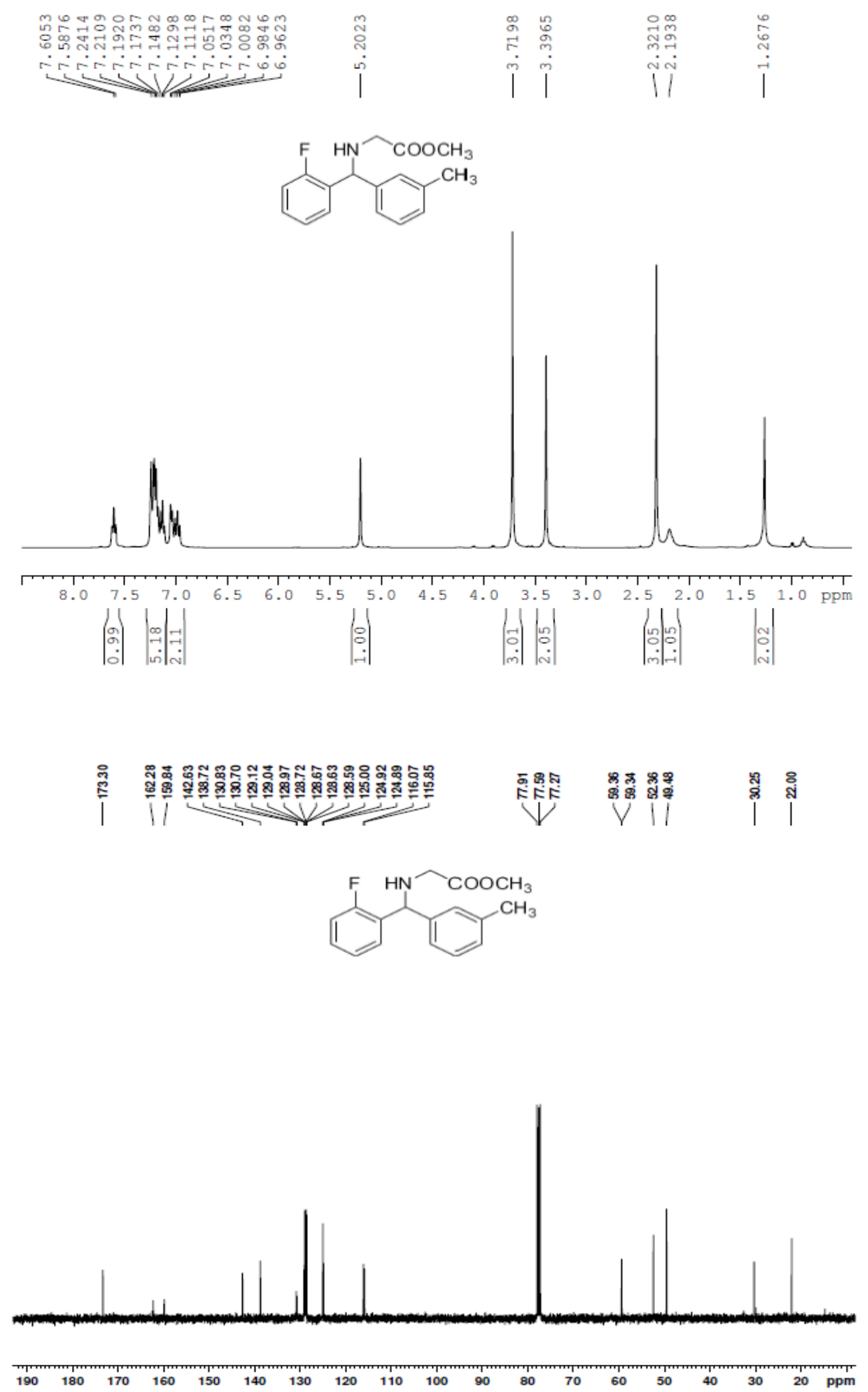


methyl 2-(((2-fluorophenyl)(4-fluorophenyl)methyl)amino)acetate (2s)
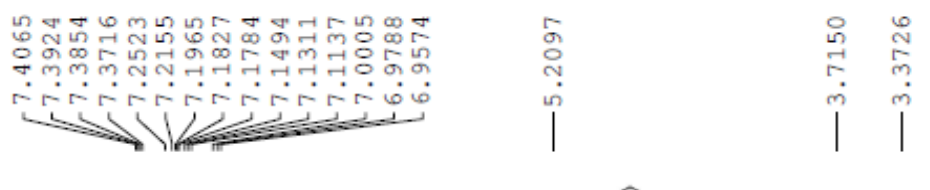

|ำ
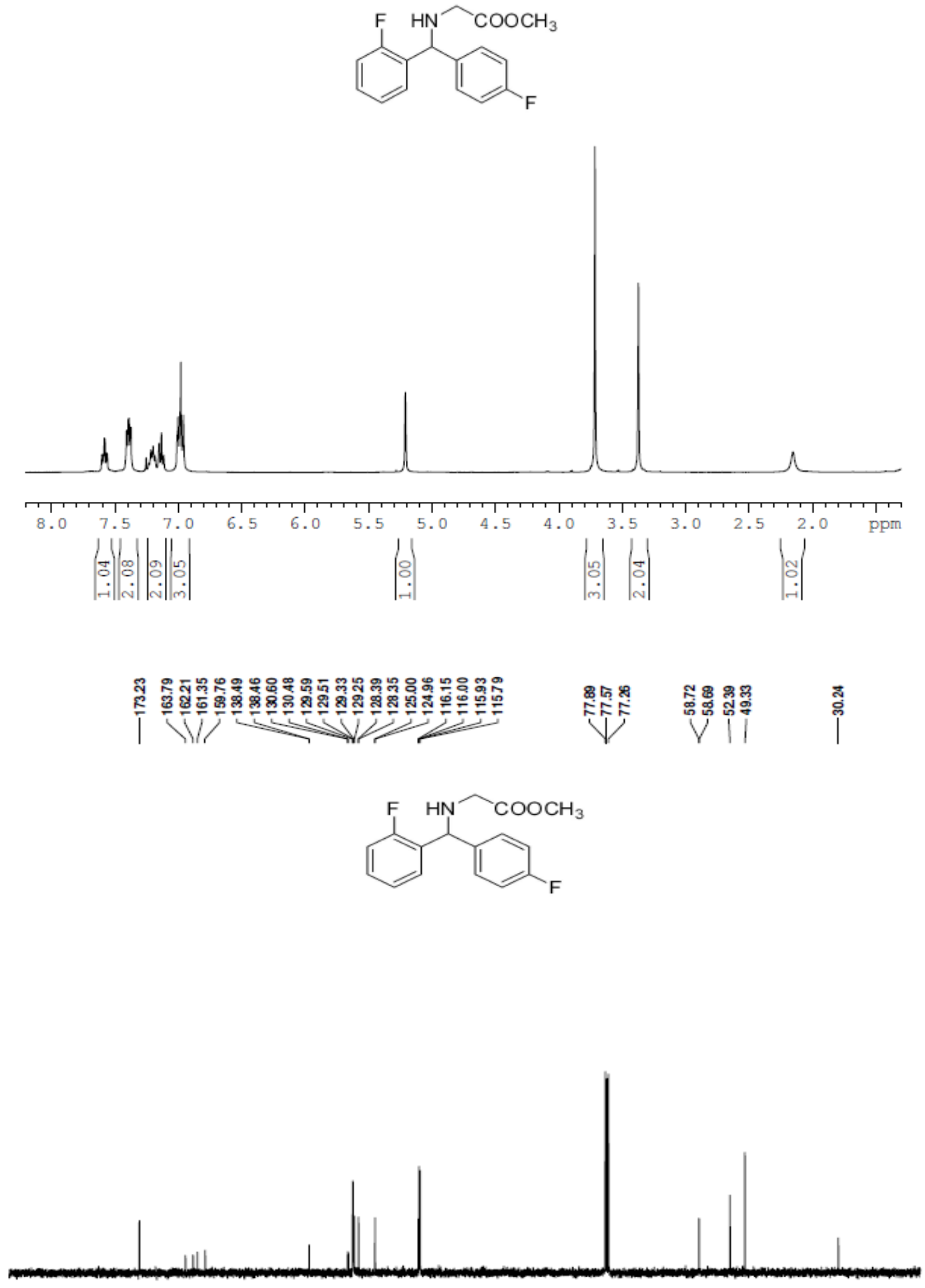

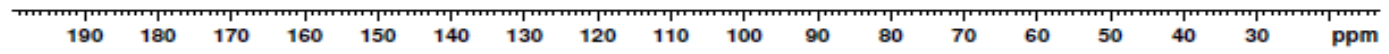

S44 
methyl 2-((naphthalen-1-yl(phenyl)methyl)amino)acetatev (2t)

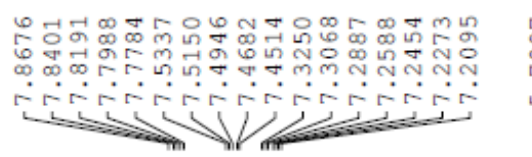

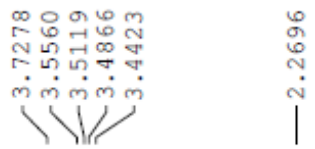<smiles>CC(=O)CNC(c1ccccc1)c1cccc2ccccc12</smiles>
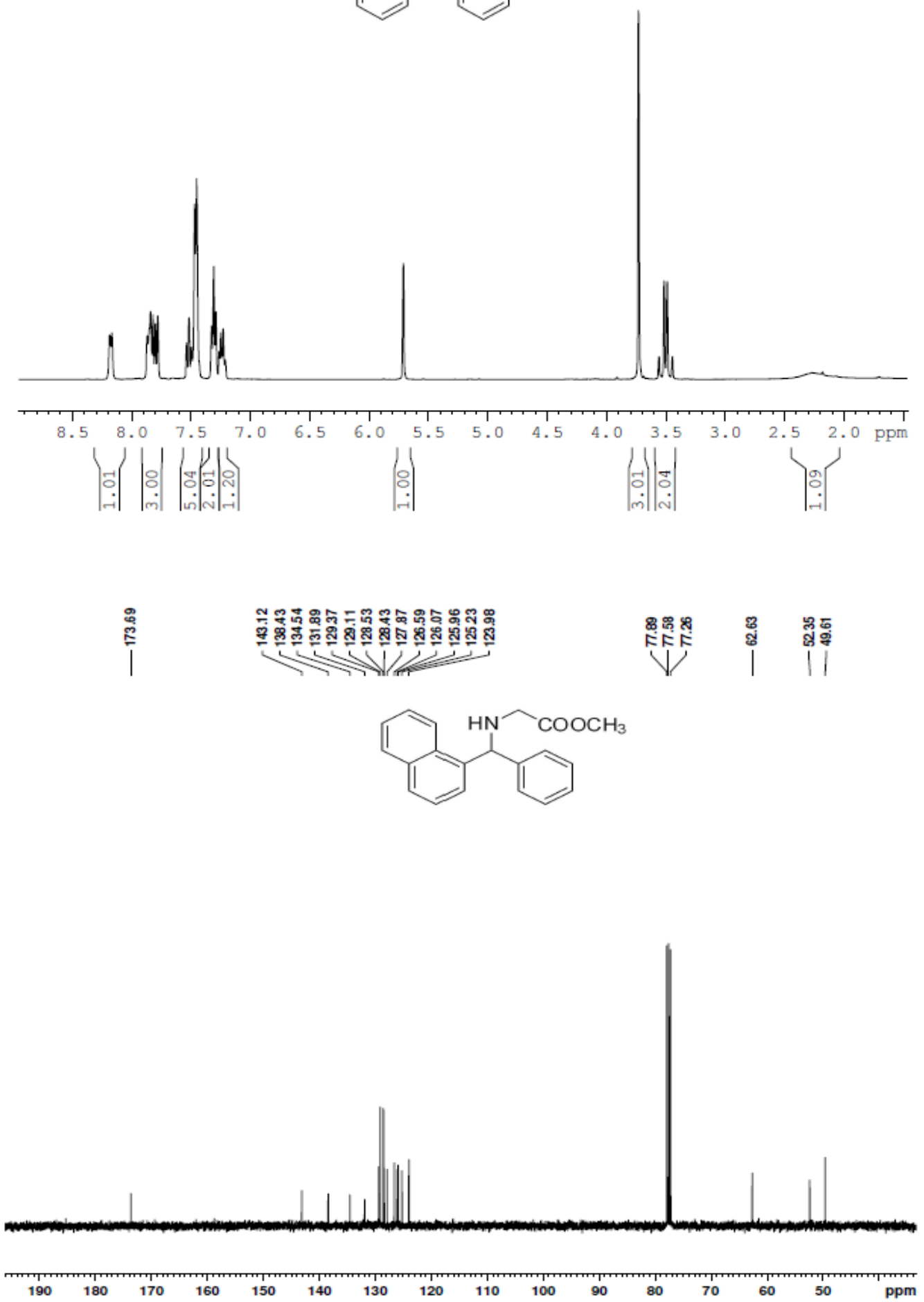
ethyl 2-((phenyl(o-tolyl)methyl)amino)acetate (2u)
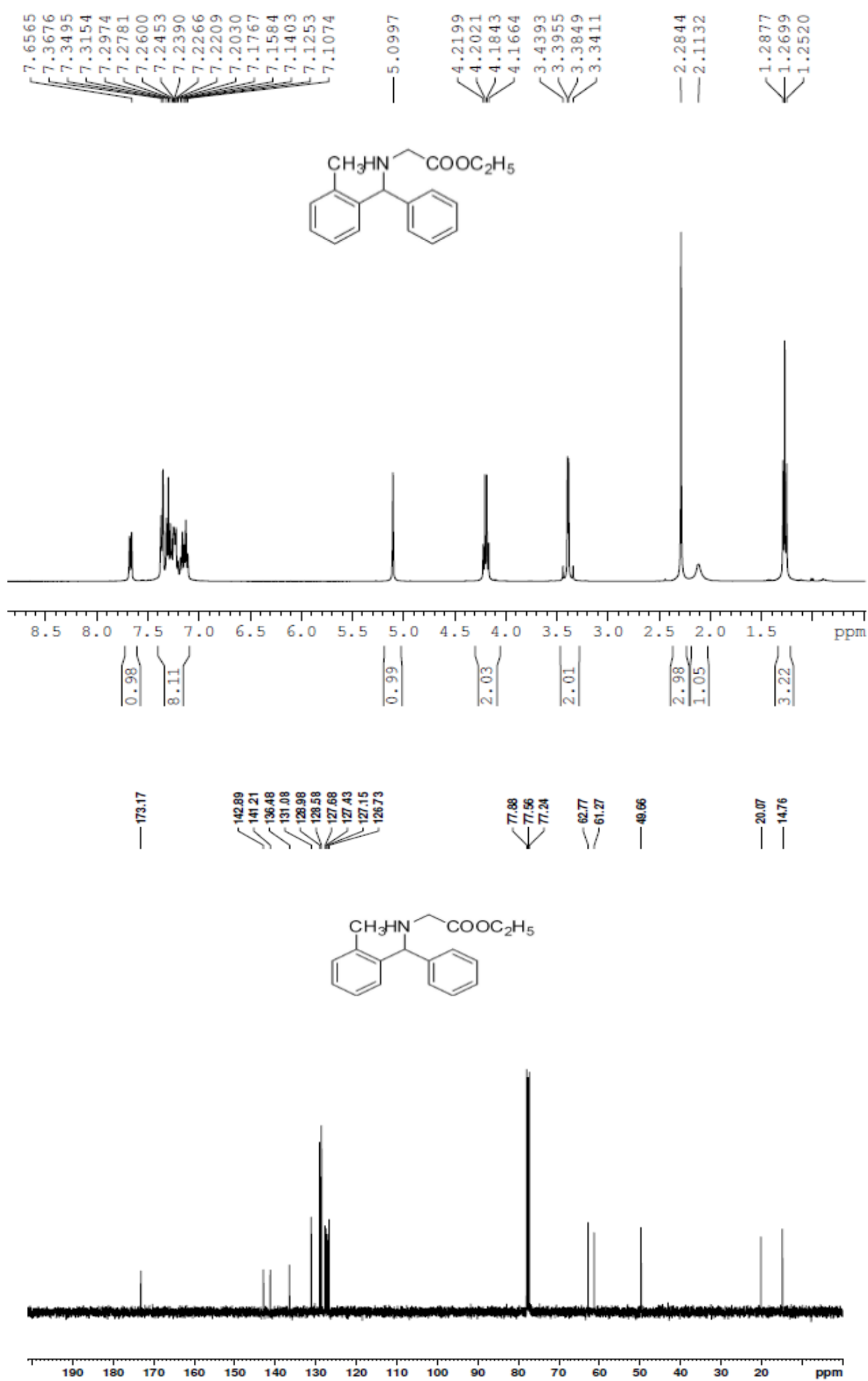
tert-butyl 2-((phenyl(o-tolyl)methyl)amino)acetate (2v)

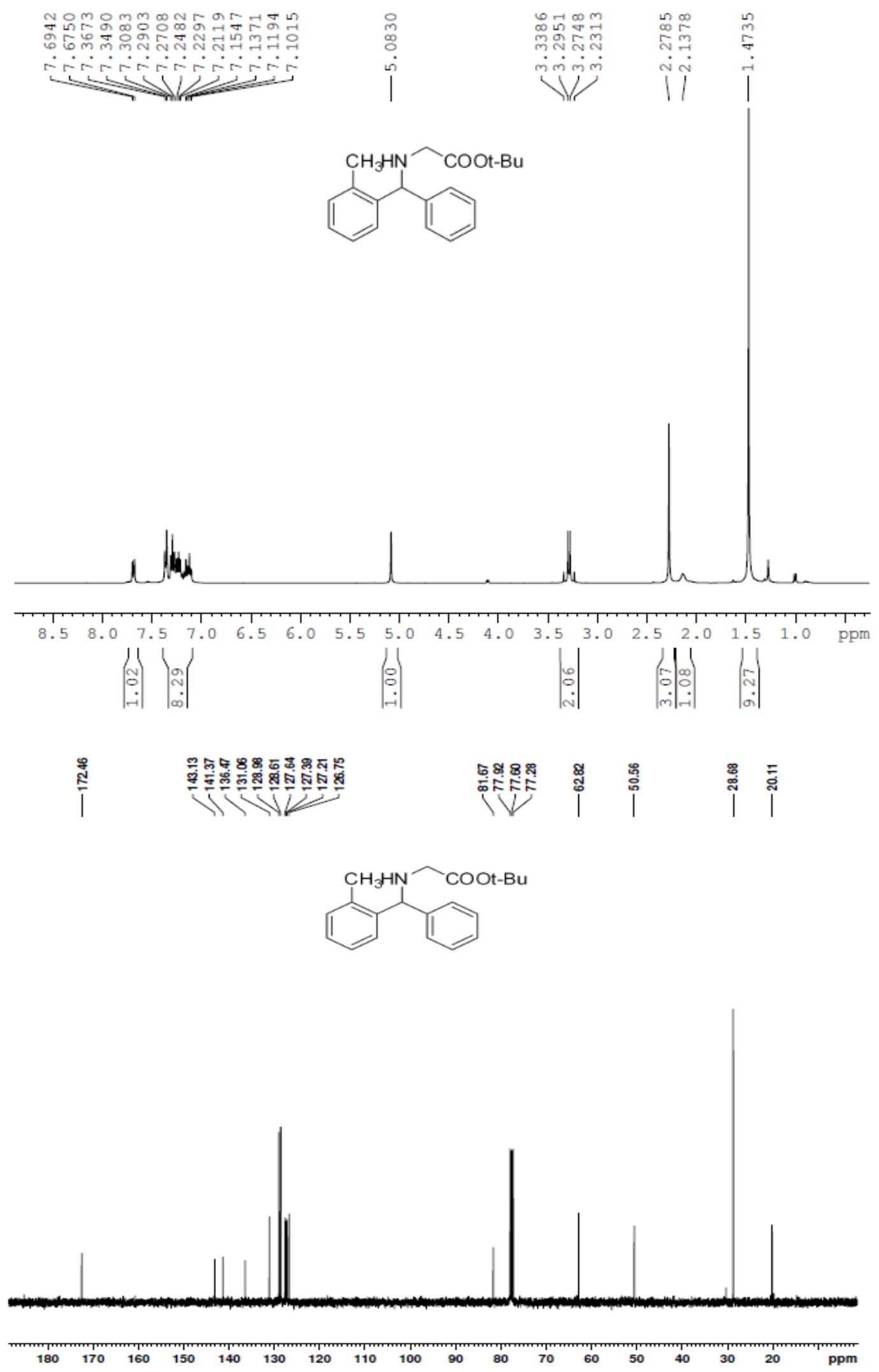


$N$-methyl-1-phenyl-1-(o-tolyl)methanamine(2w)

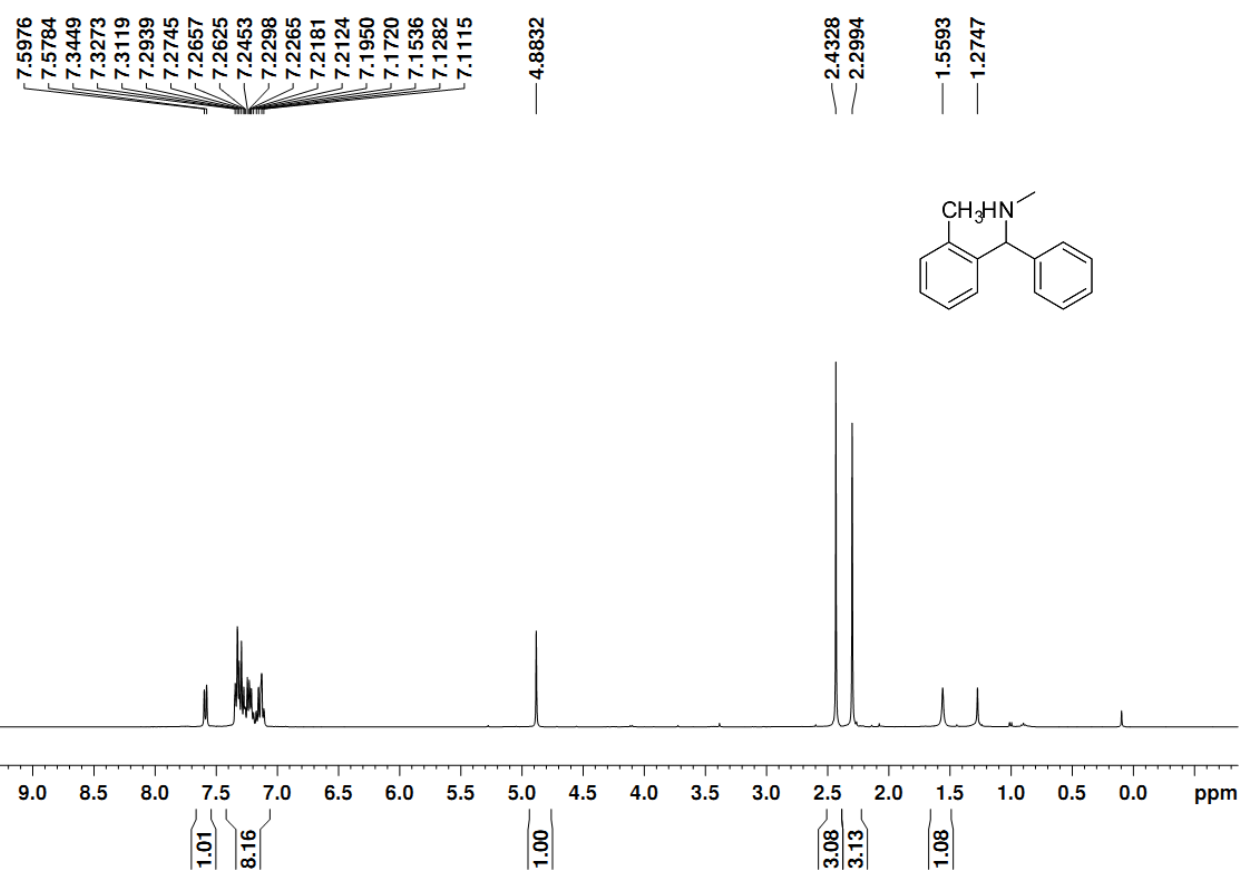

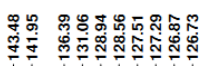

你

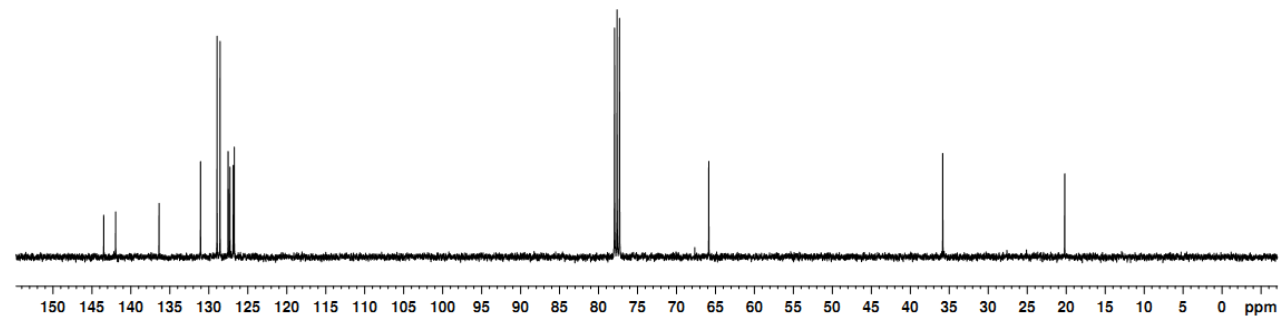


$N$-(phenyl(o-tolyl)methyl)ethanamine(2x)

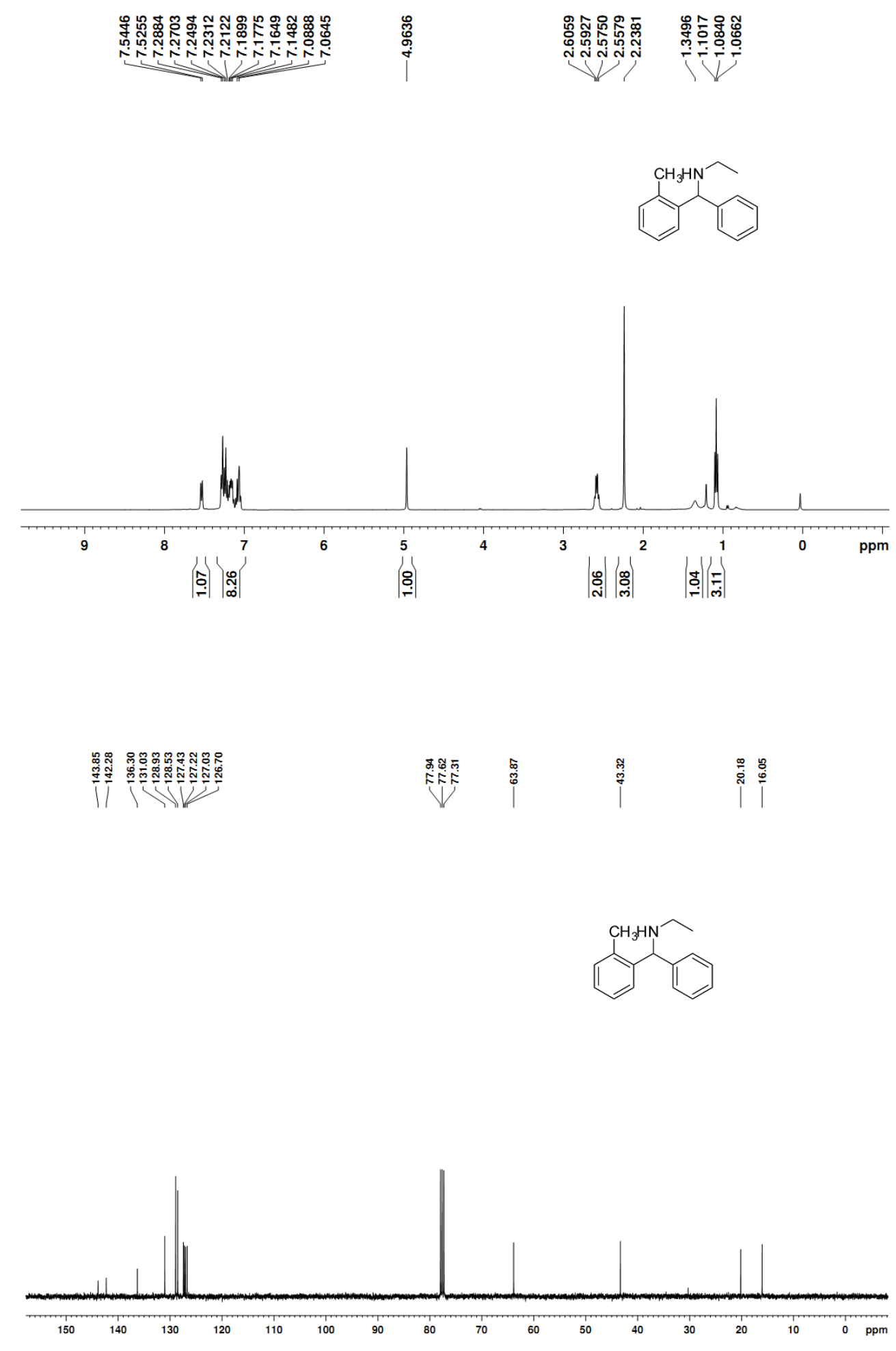


methyl 2-((phenyl(m-tolyl)methyl)amino)acetate (3a)
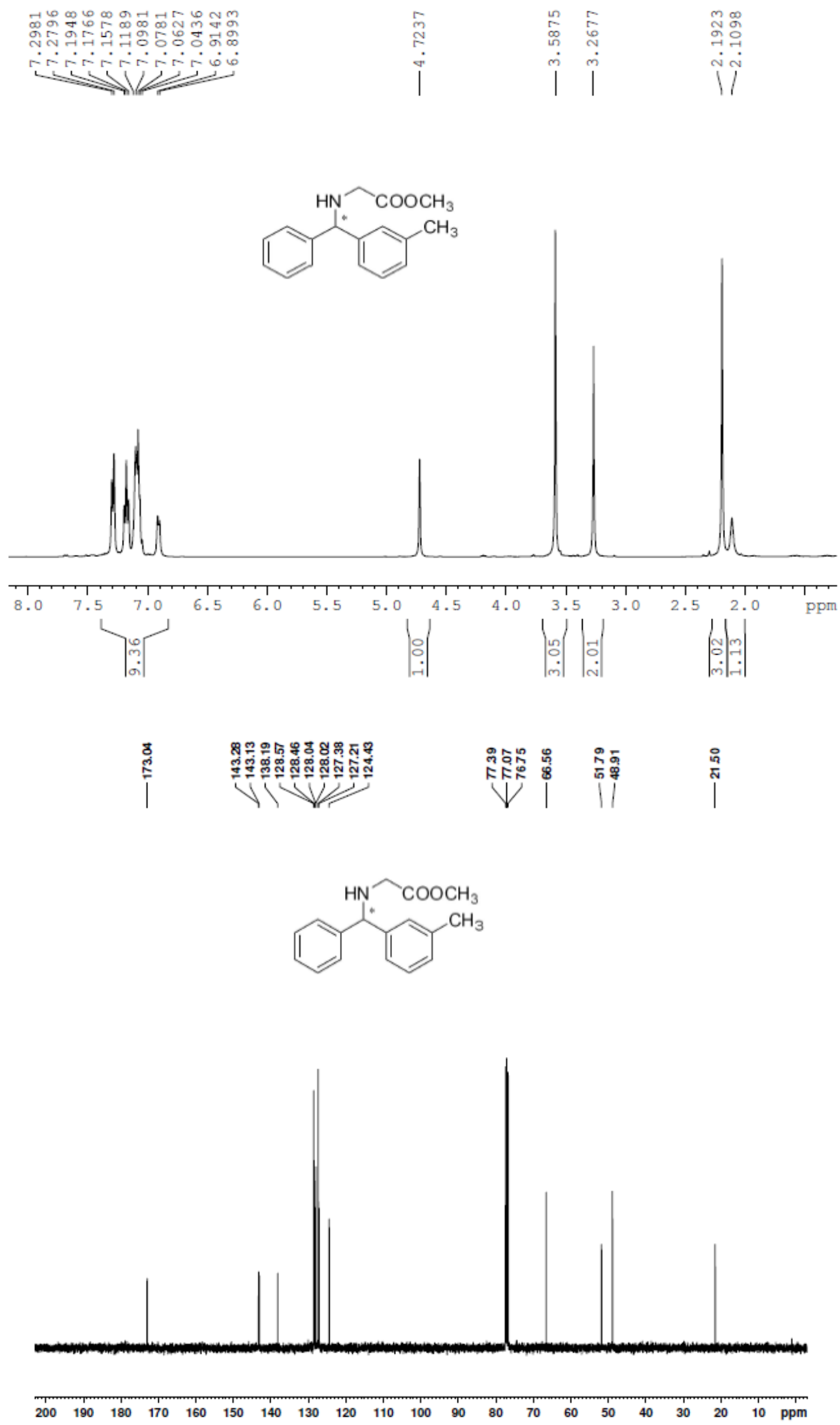

S50 
methyl 2-((phenyl(p-tolyl)methyl)amino)acetate (3b)
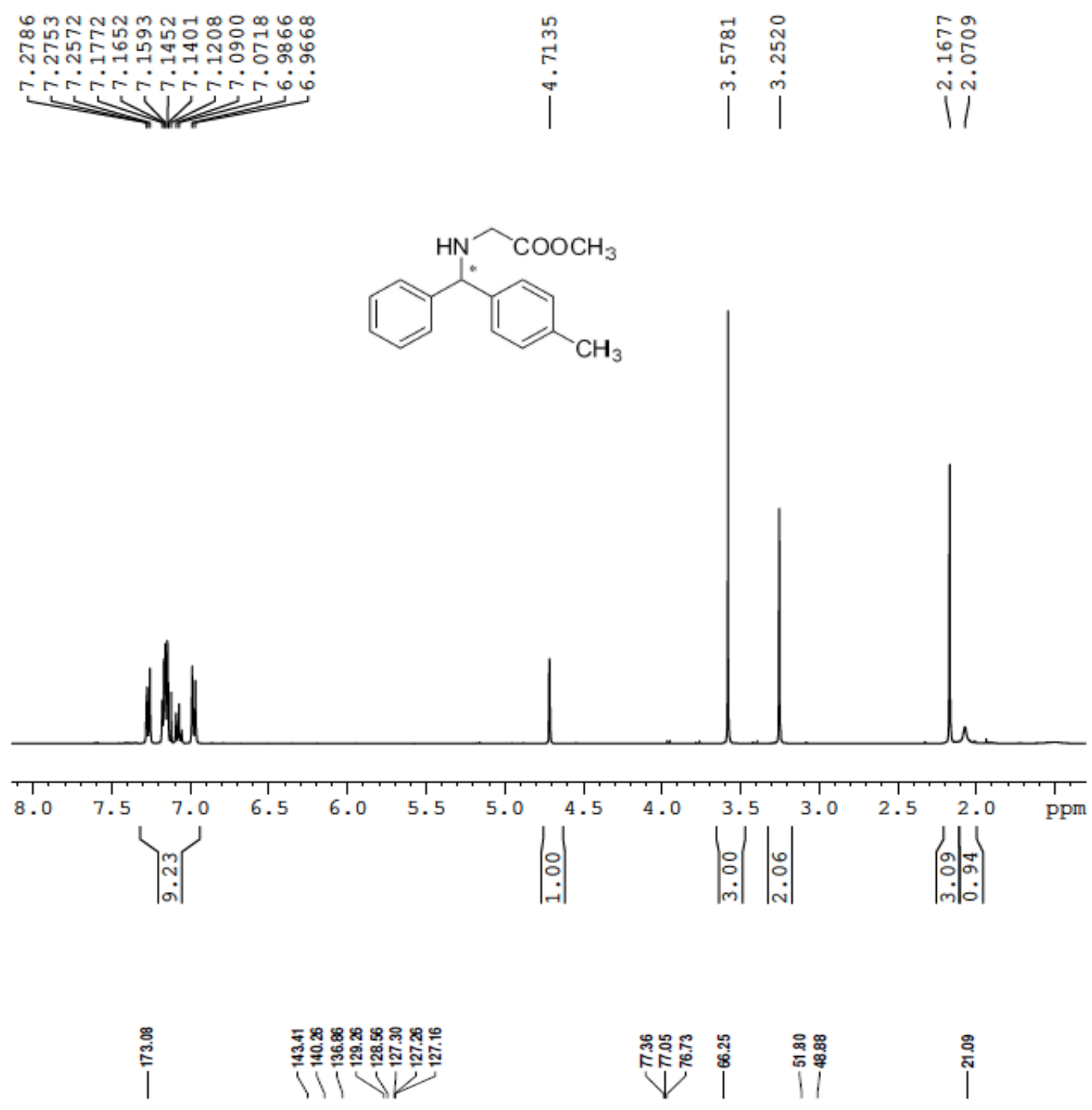

î
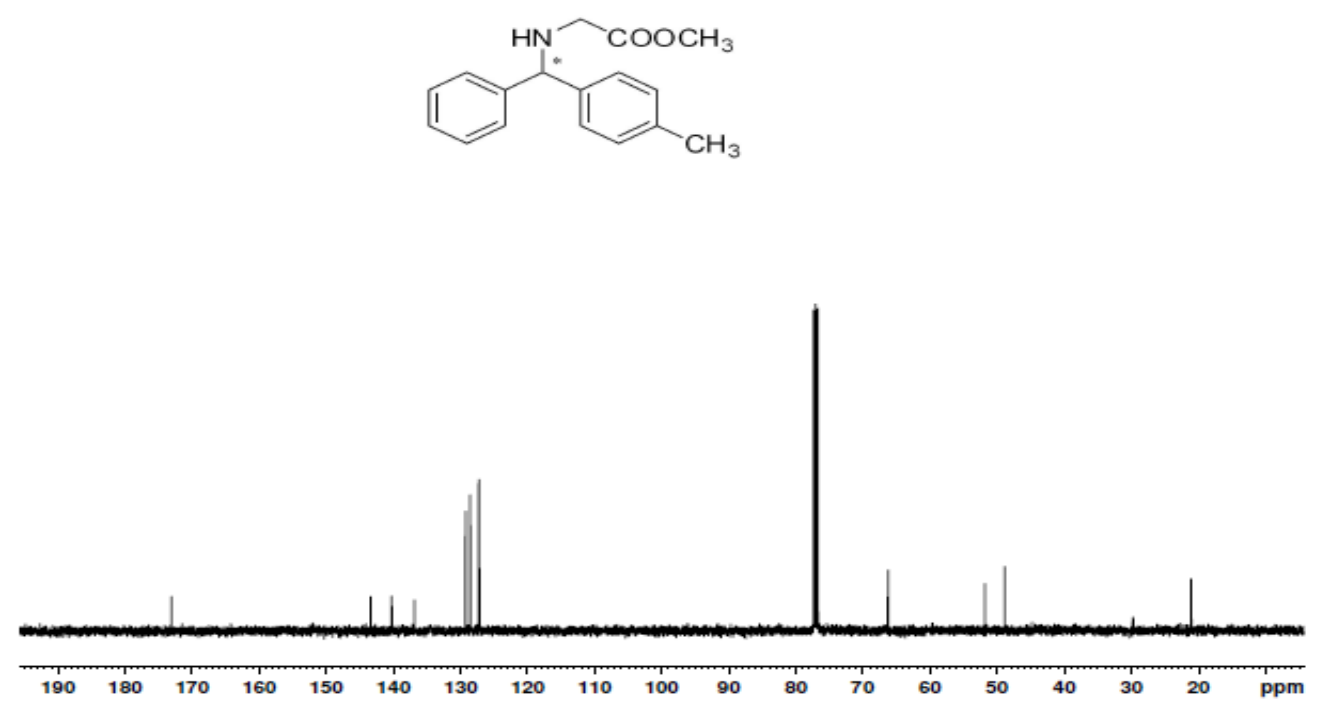

S51 
methyl 2-(((3-fluorophenyl)(phenyl)methyl)amino)acetate (3c)
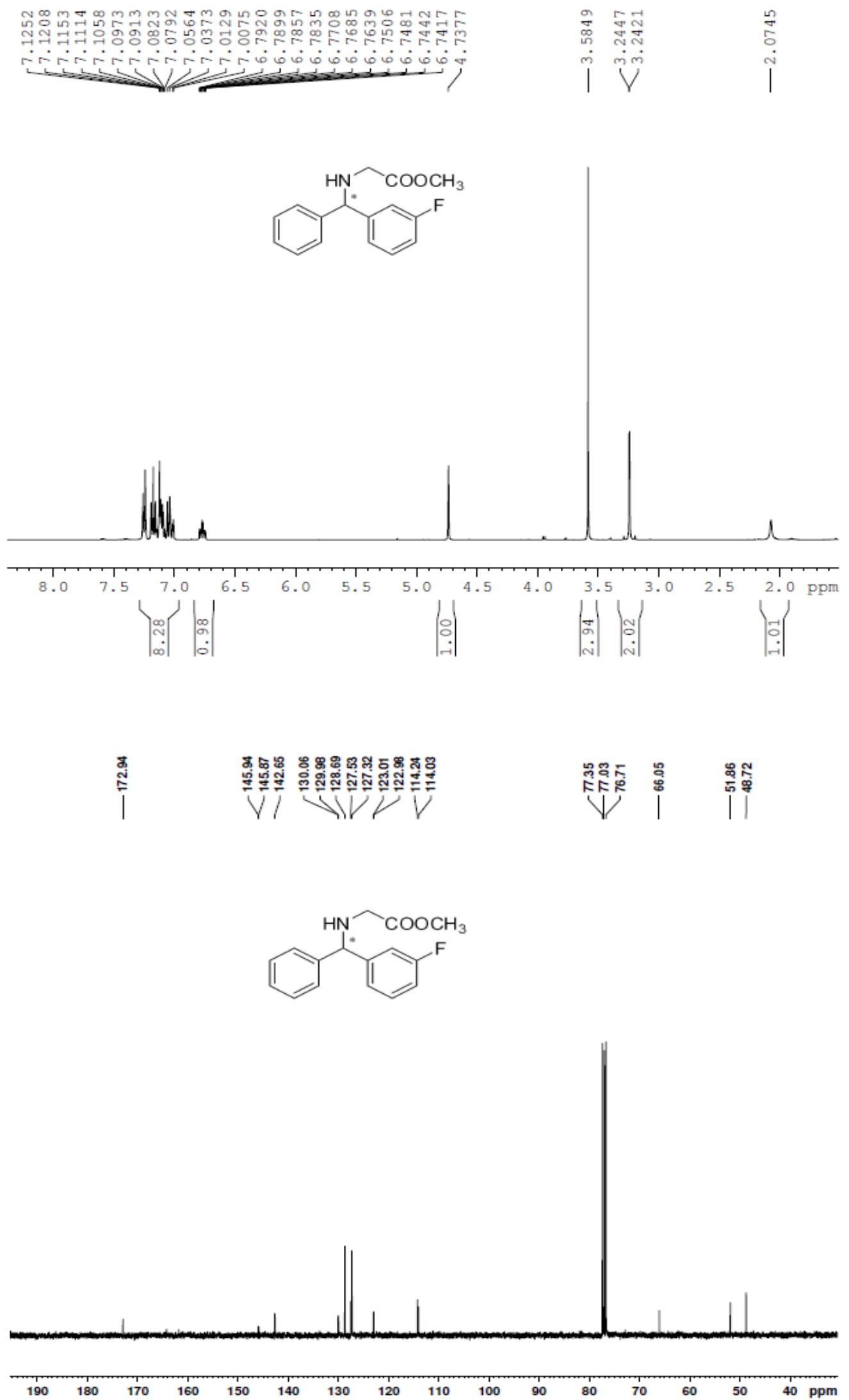
methyl 2-(((4-fluorophenyl)(phenyl)methyl)amino)acetate (3d)

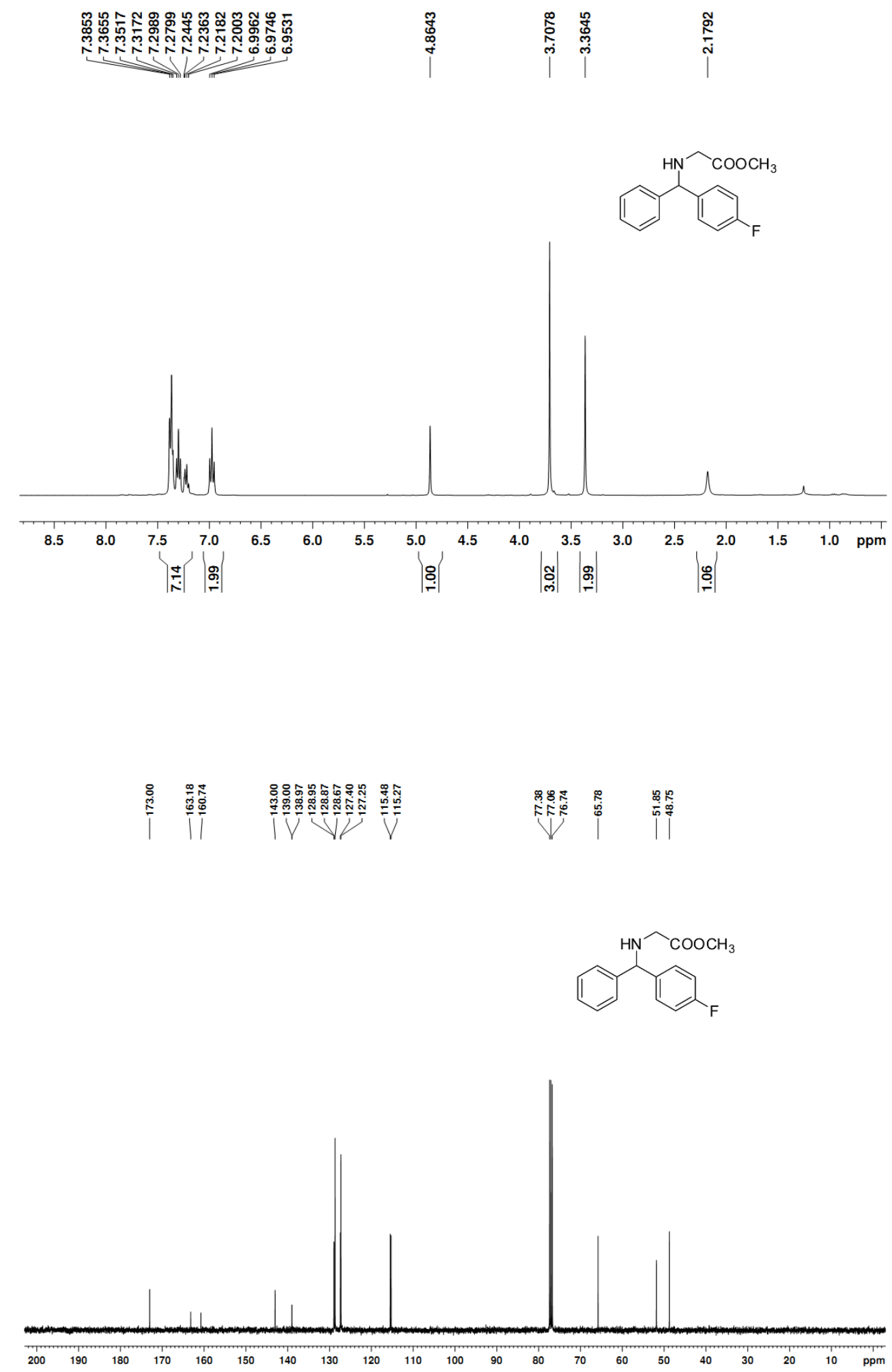


methyl 2-(((4-chlorophenyl)(phenyl)methyl)amino)acetate (3e)
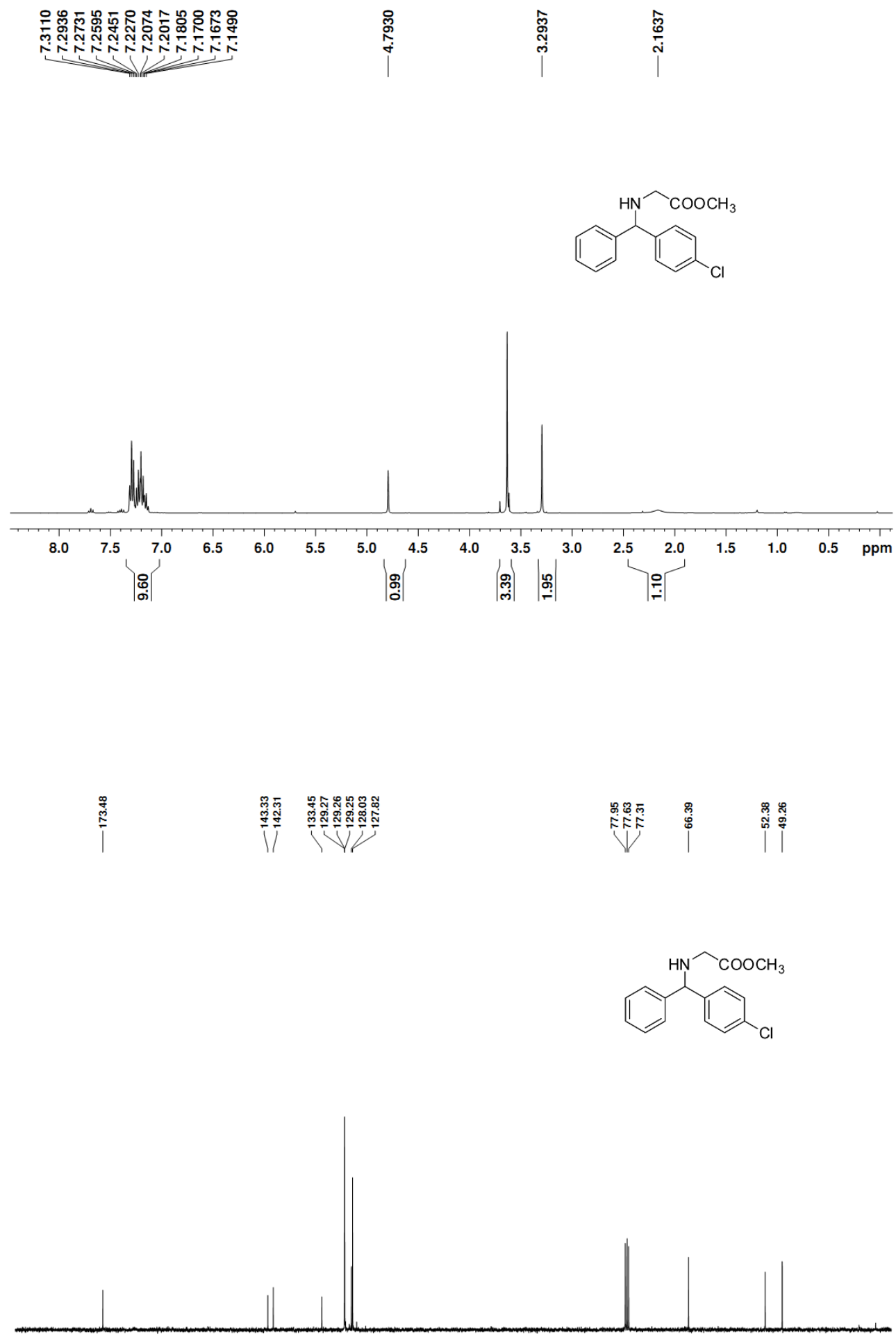

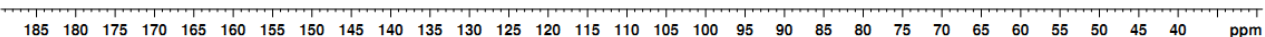


ethyl 2-(((4-chlorophenyl)(phenyl)methyl)(2-methoxy-2-oxoethyl)amino)acetate (4)
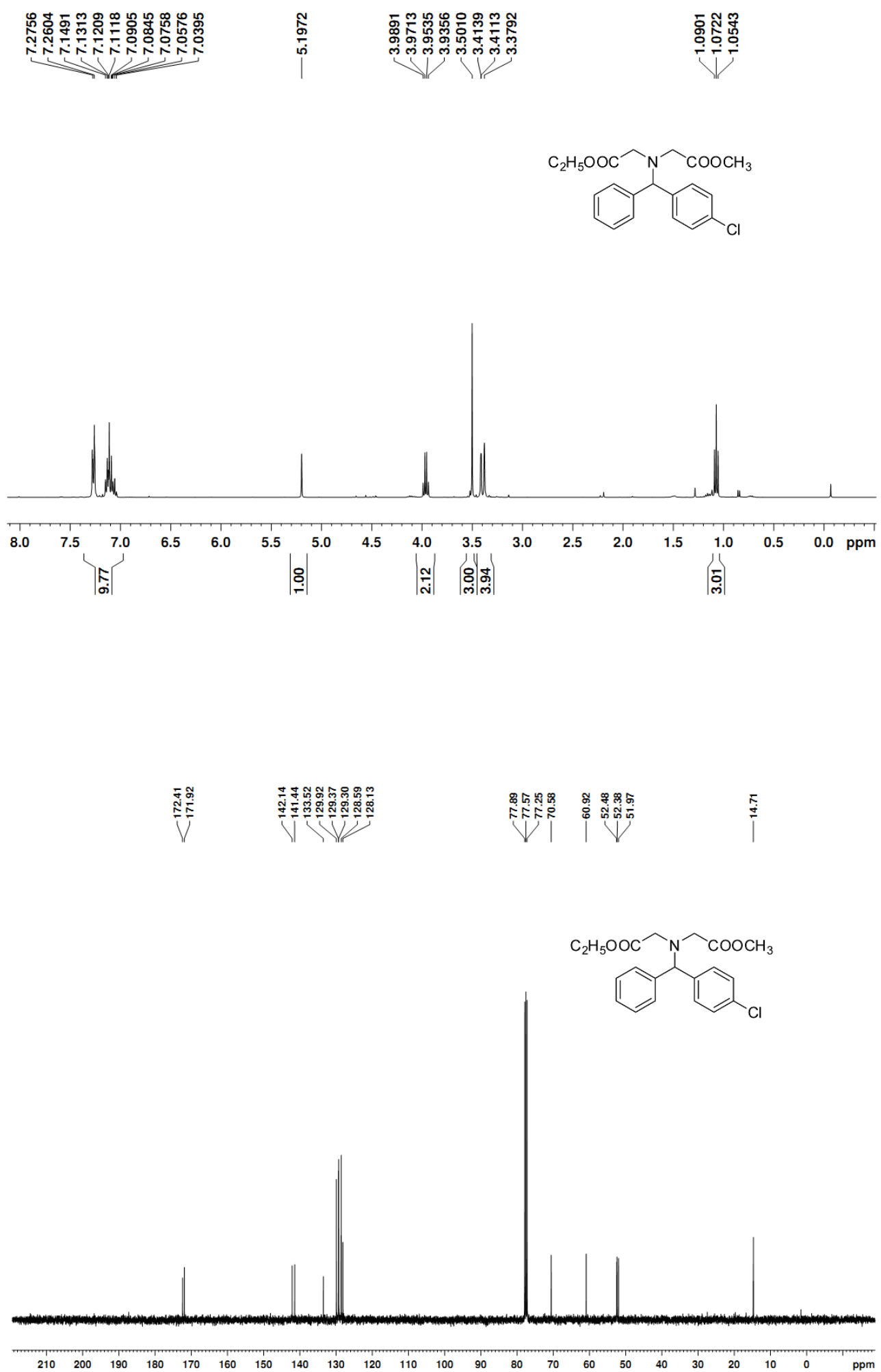
phenyl(o-tolyl)methanamine (5a)

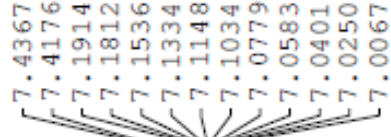

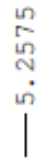

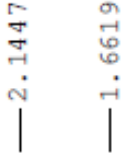<smiles>Cc1ccccc1C(N)c1ccccc1</smiles>
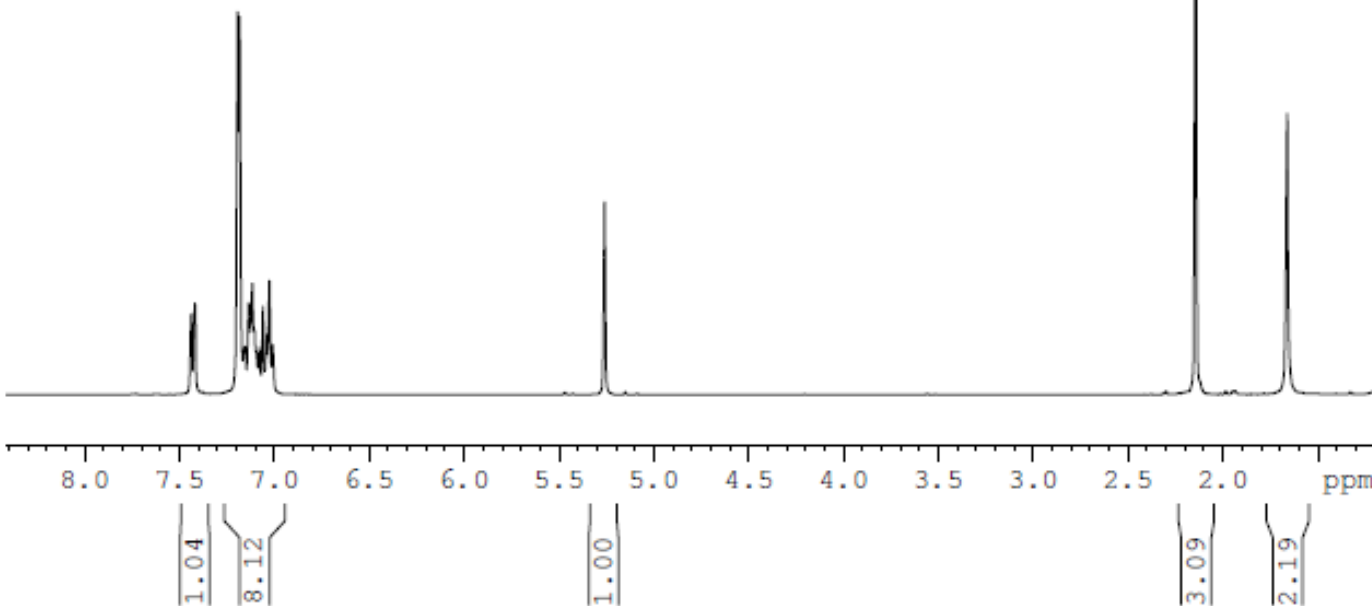

(4-chlorophenyl)(phenyl)methanamine (5b)

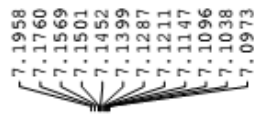

$\left.\right|_{\substack{0 \\ 0}} ^{\dot{0}}$
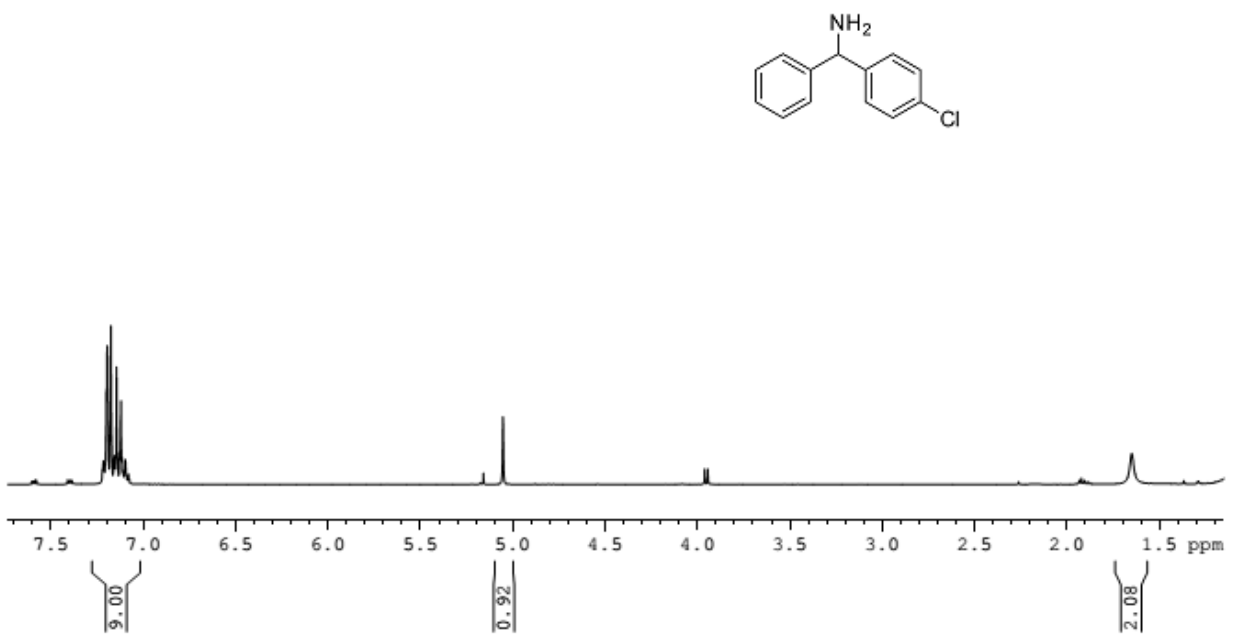

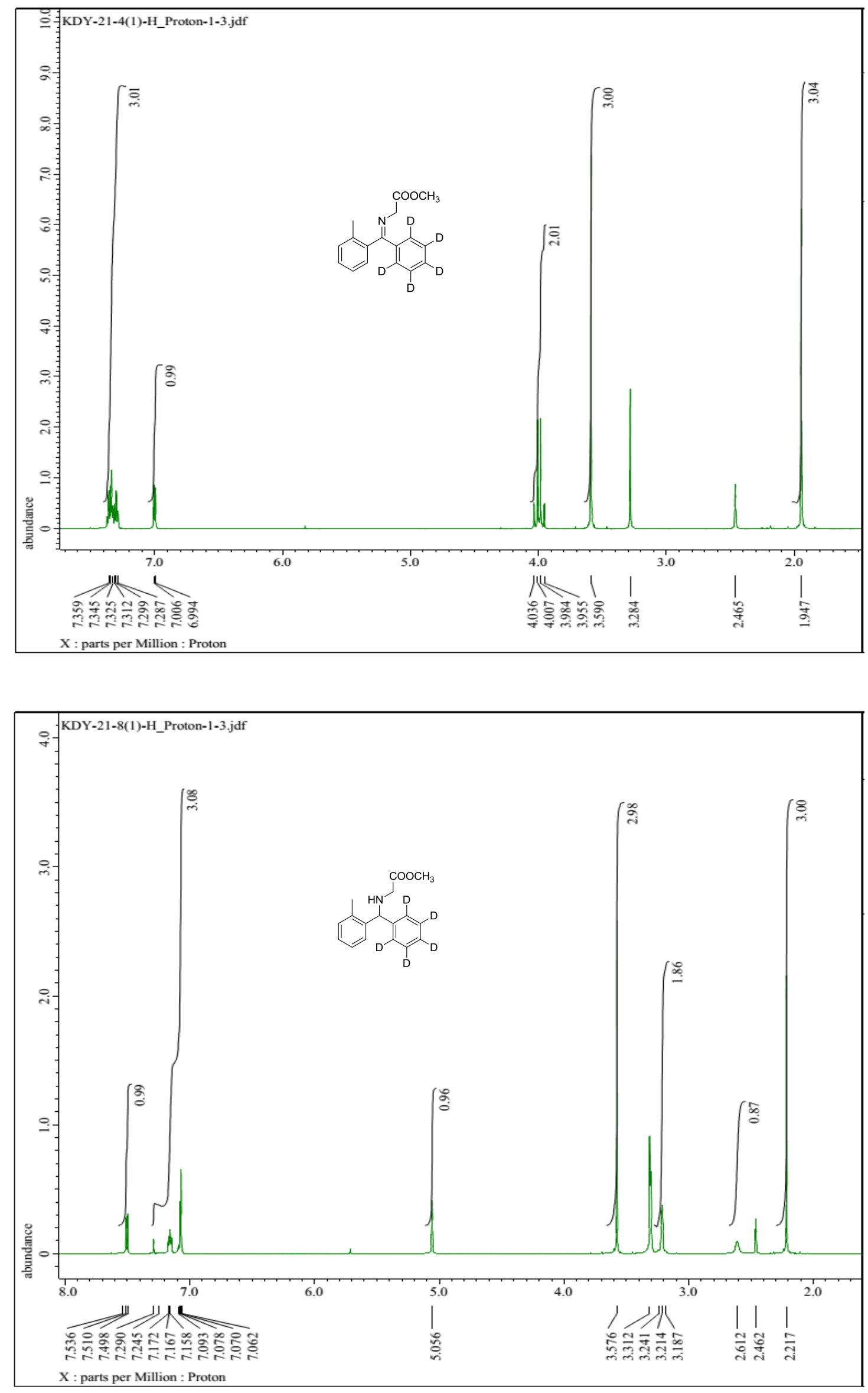


\section{HPLC spectra}

\section{methyl 2-((phenyl(o-tolyl)methyl)amino)acetate (2a)}
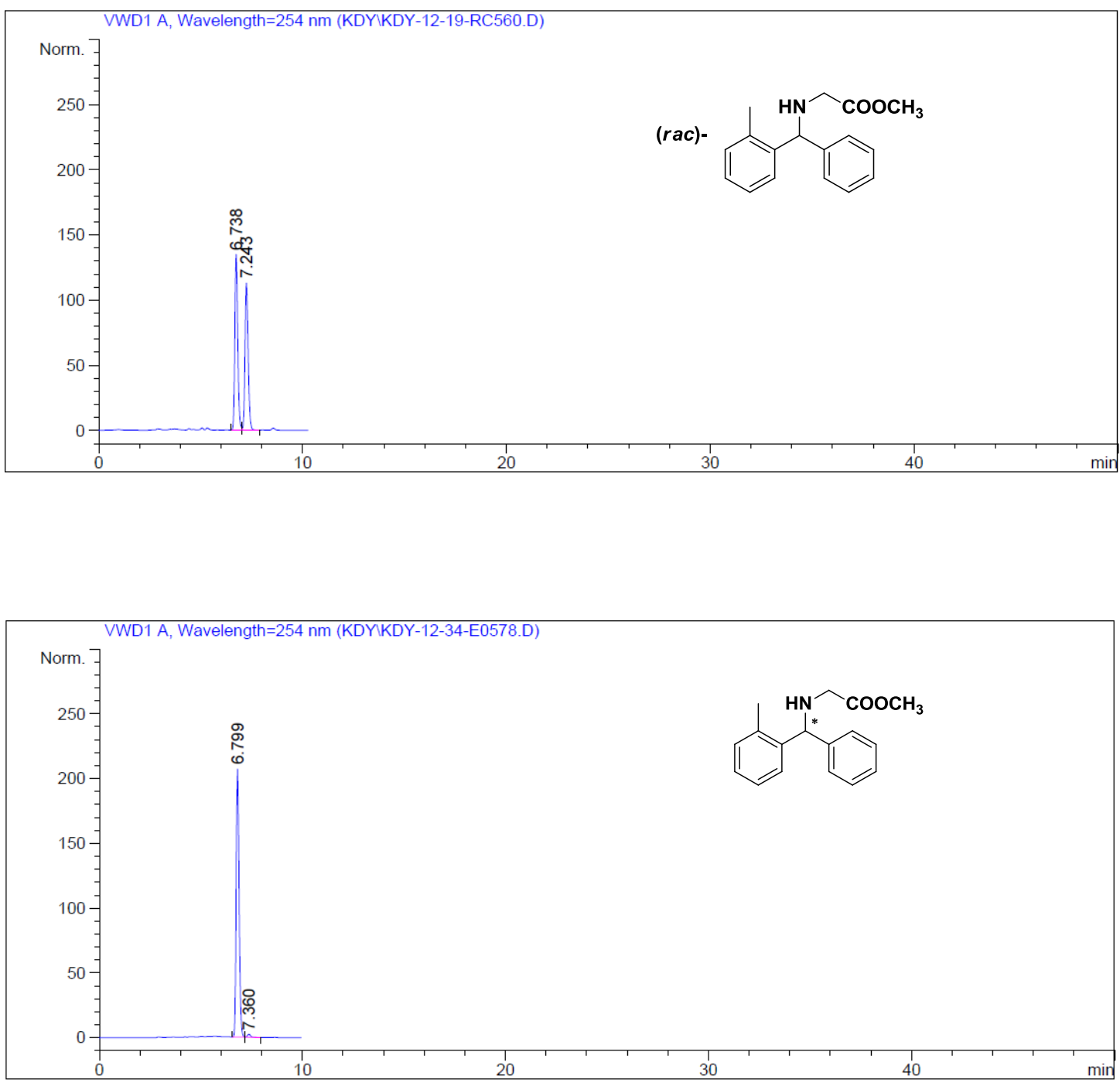

\begin{tabular}{|c|c|c|c|c|c|c|}
\hline $\begin{array}{c}\text { Peak } \\
\#\end{array}$ & $\begin{array}{c}\text { RetTime } \\
\text { [min] }\end{array}$ & Type & $\begin{array}{l}\text { Width } \\
\text { [min] }\end{array}$ & $\begin{array}{c}\text { Area } \\
{\left[\mathrm{mAU}^{*} \mathrm{~S}\right]}\end{array}$ & $\begin{array}{l}\text { Height } \\
{[\mathrm{mAU}]}\end{array}$ & $\begin{array}{c}\text { Area } \\
\text { 응 }\end{array}$ \\
\hline & & & & ---------- & ---------- & $--------\mid$ \\
\hline 1 & 6.799 & $B B$ & 0.1568 & 2088.47949 & 206.86049 & 9082 \\
\hline 2 & 7.360 & $B B$ & & & 9457 & 0918 \\
\hline
\end{tabular}

Totals :

$2111.53247 \quad 209.05506$ 
methyl 2-(((3-methoxyphenyl)(o-tolyl)methyl)amino)acetate (2b)
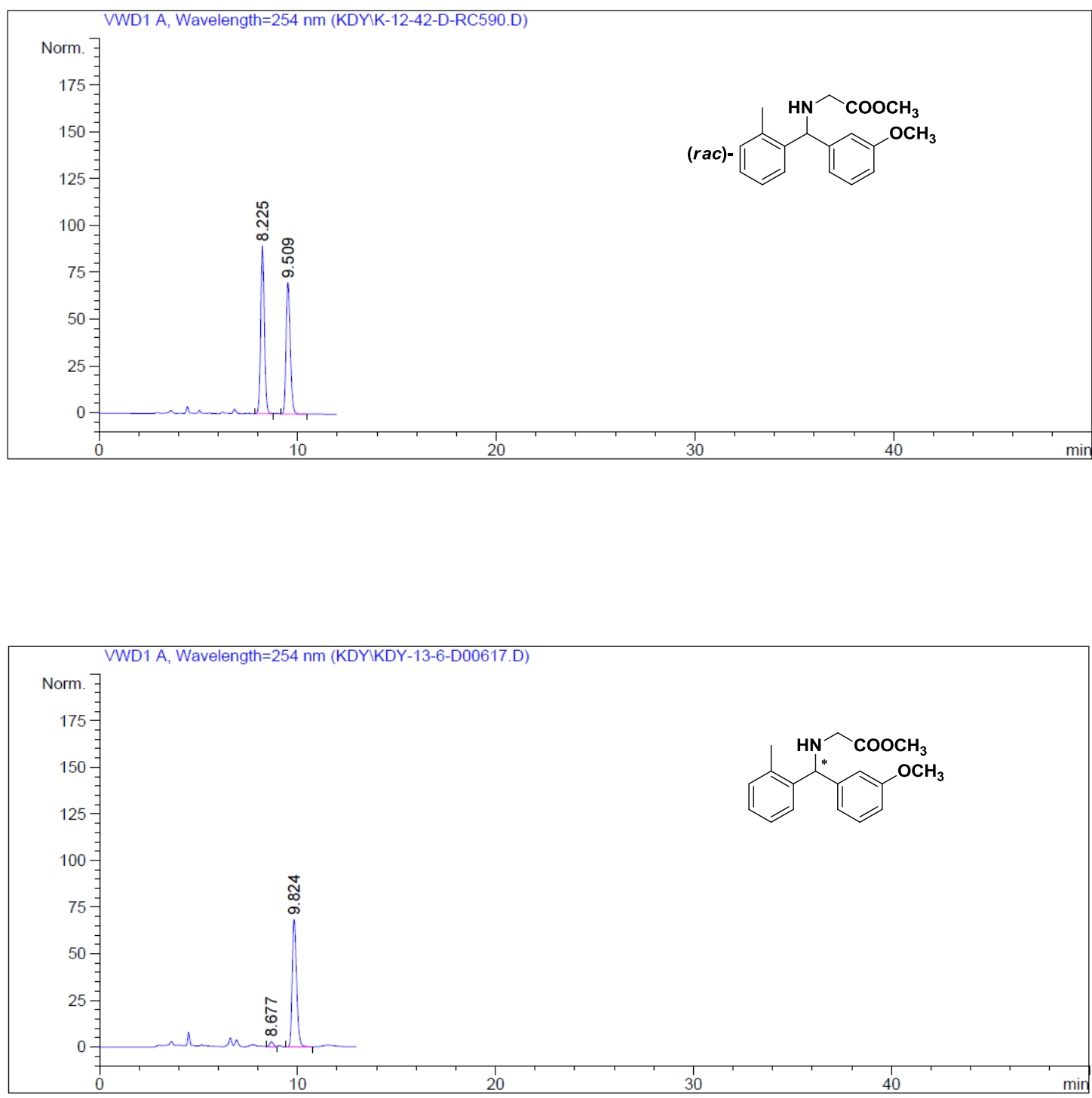

\begin{tabular}{|c|c|c|c|c|c|c|}
\hline $\begin{array}{c}\text { Peak } \\
\quad \#\end{array}$ & $\begin{array}{c}\text { RetTime } \\
\text { [min] }\end{array}$ & Type & $\begin{array}{c}\text { Width } \\
\text { [min] }\end{array}$ & $\begin{array}{c}\text { Area } \\
{\left[\mathrm{mAU}^{*} \mathrm{~S}\right]}\end{array}$ & $\begin{array}{l}\text { Height } \\
{[\mathrm{mAU}]}\end{array}$ & $\begin{array}{c}\text { Area } \\
\text { 응 }\end{array}$ \\
\hline 1 & 8.677 & BB & 0.1907 & 31.94342 & 2.63069 & 2.9455 \\
\hline 2 & 9.824 & $\mathrm{BB}$ & 0.2379 & 1052.52441 & 68.25546 & 97.0545 \\
\hline otal & Ls: & & & 1084.46783 & 70.88615 & \\
\hline
\end{tabular}


methyl 2-((m-tolyl $(o$-tolyl $)$ methyl $)$ amino $)$ acetate (2c)
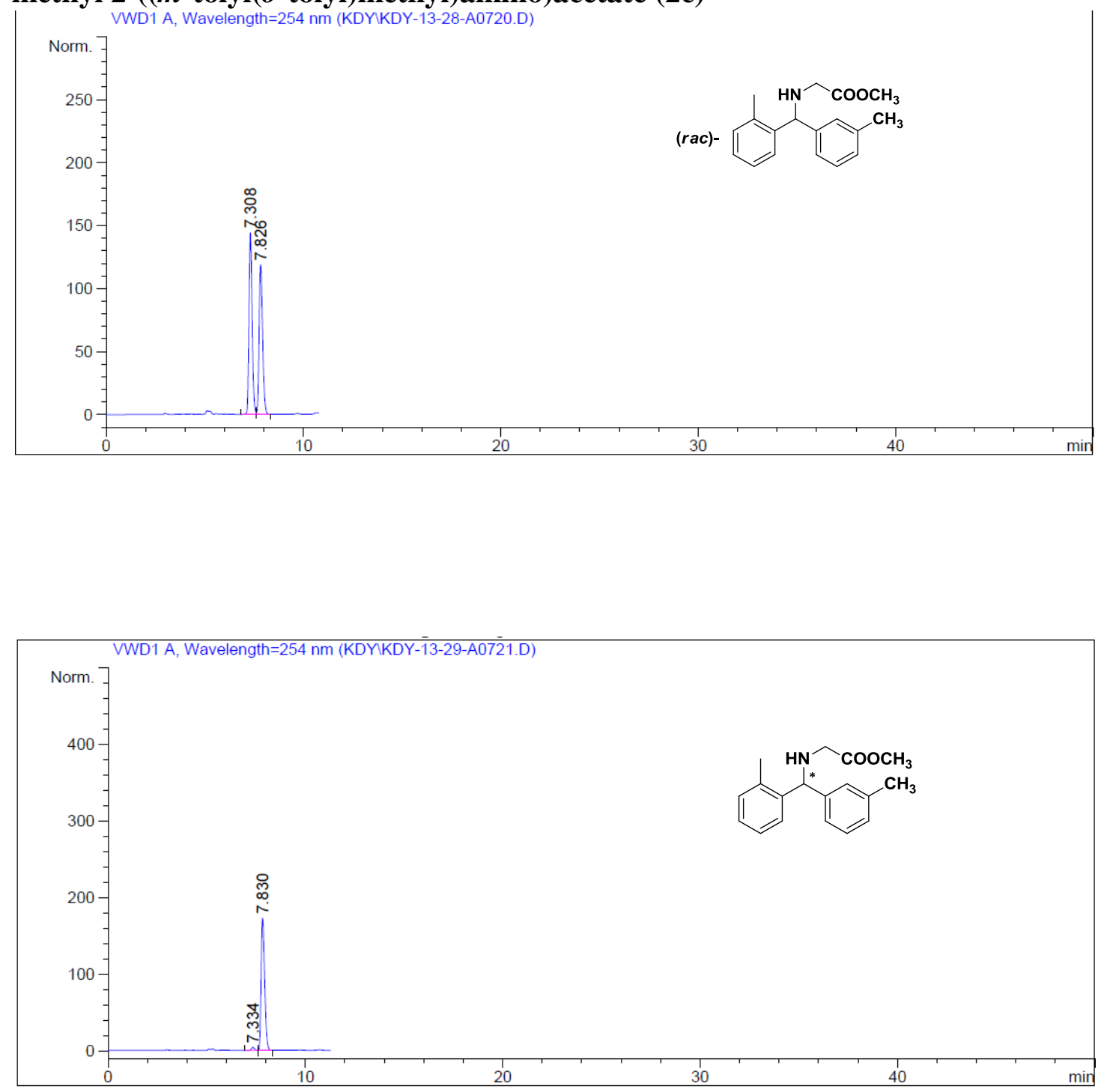

\begin{tabular}{|c|c|c|c|c|c|c|}
\hline $\begin{array}{c}\text { Peak } \\
\quad \#\end{array}$ & $\begin{array}{c}\text { RetTime } \\
\text { [min] }\end{array}$ & Type & $\begin{array}{l}\text { Width } \\
{[\mathrm{min}]}\end{array}$ & $\begin{array}{c}\text { Area } \\
{\left[\mathrm{mAU}^{*} \mathrm{~s}\right]}\end{array}$ & $\begin{array}{l}\text { Height } \\
\text { [mAU] }\end{array}$ & $\begin{array}{c}\text { Area } \\
\quad \frac{\circ}{0}\end{array}$ \\
\hline 1 & 7.334 & BB & 0.1653 & 41.61227 & 3.90697 & 1.9063 \\
\hline 2 & 7.830 & $\mathrm{BB}$ & 0.1928 & 2141.28003 & 172.55591 & 98.0937 \\
\hline Total & $\perp S$ & & & 2182.89230 & 176.46287 & \\
\hline
\end{tabular}


methyl 2-((o-tolyl(p-tolyl)methyl)amino)acetate (2d)
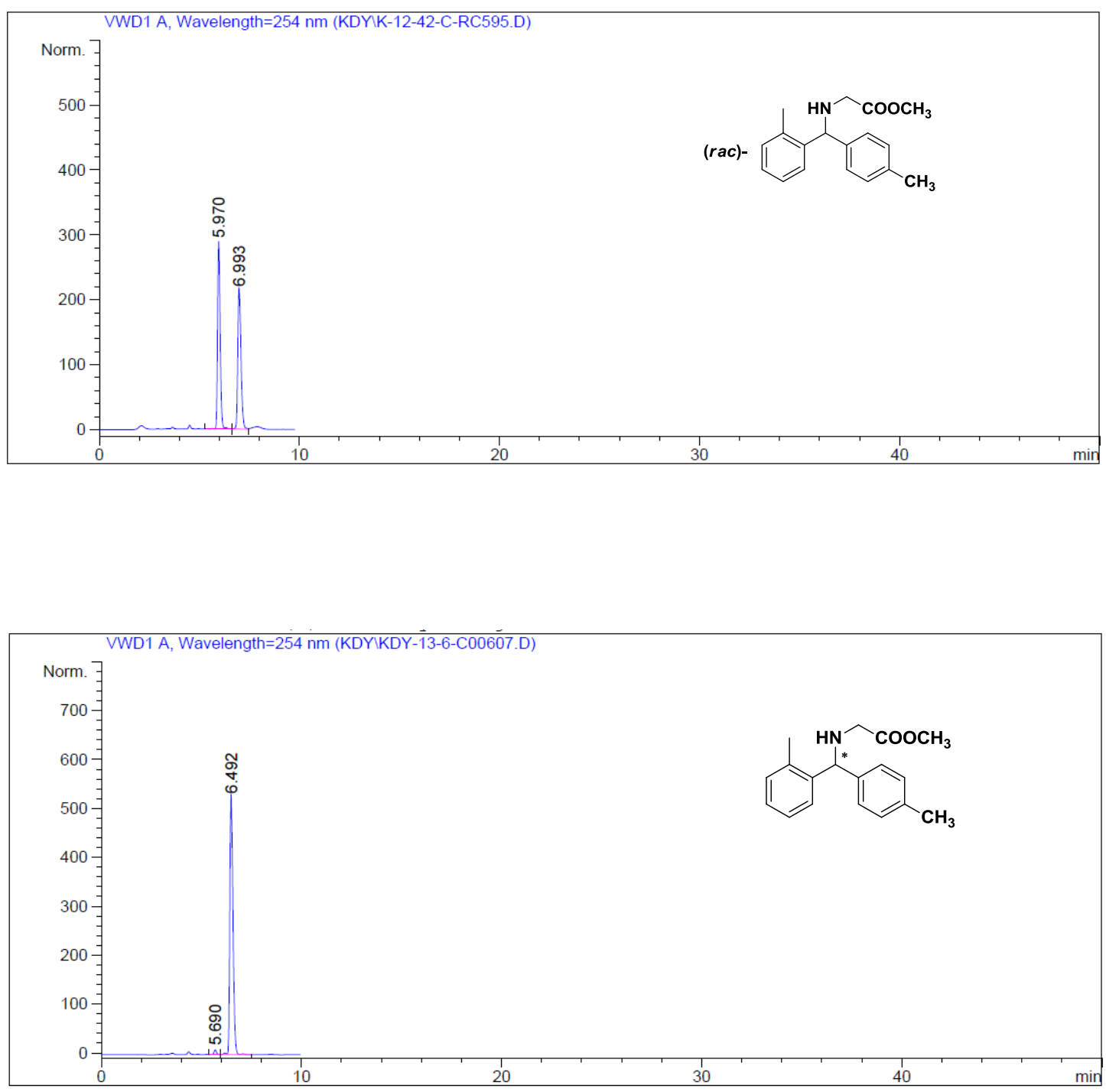

\begin{tabular}{|c|c|c|c|c|c|c|}
\hline $\begin{array}{c}\text { Peak } \\
\quad \#\end{array}$ & $\begin{array}{c}\text { RetTime } \\
\text { [min] }\end{array}$ & Type & $\begin{array}{l}\text { Width } \\
\text { [min] }\end{array}$ & $\begin{array}{c}\text { Area } \\
{\left[\mathrm{mAU}{ }^{*} \mathrm{~s}\right]}\end{array}$ & $\begin{array}{l}\text { Height } \\
\text { [mAU] }\end{array}$ & $\begin{array}{c}\text { Area } \\
\frac{\circ}{0}\end{array}$ \\
\hline- & $2-2$ & & & ---------- & & ----- \\
\hline 1 & 5.690 & BB & 0.1425 & 83.04182 & 9.45085 & 1.4001 \\
\hline 2 & 6.492 & BB & 0.1860 & 5847.93555 & 524.84955 & 98.5999 \\
\hline ot & & & & 5930.97737 & 534.30040 & \\
\hline
\end{tabular}


methyl 2-(((4-fluorophenyl)(o-tolyl)methyl)amino)acetate (2e)
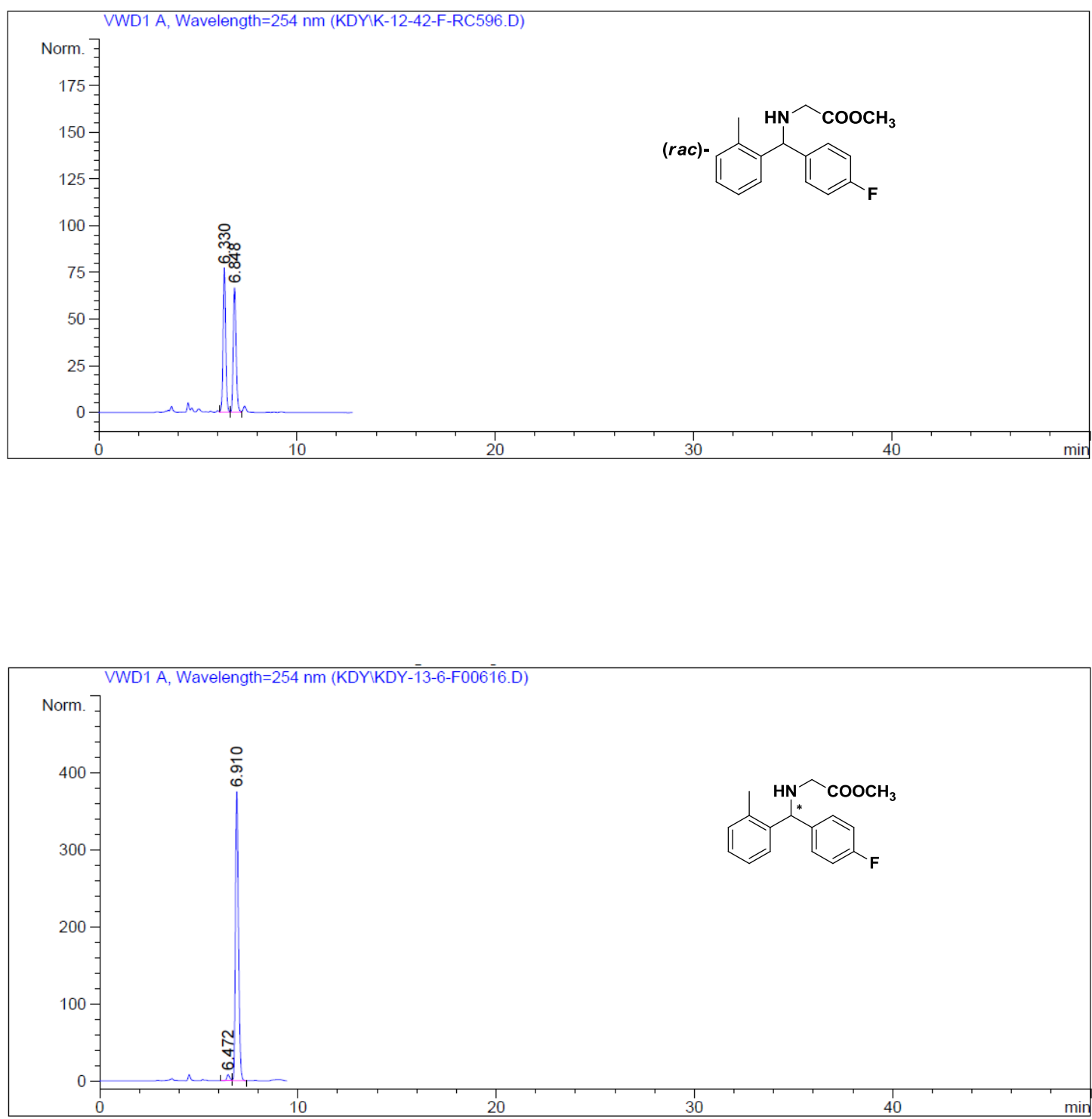

\begin{tabular}{|c|c|c|c|c|c|c|}
\hline $\begin{array}{c}\text { Peak } \\
\quad \#\end{array}$ & $\begin{array}{c}\text { RetTime } \\
\text { [min] }\end{array}$ & Type & $\begin{array}{l}\text { Width } \\
\text { [min] }\end{array}$ & $\begin{array}{c}\text { Area } \\
{\left[\mathrm{mAU}^{*} \mathrm{~S}\right]}\end{array}$ & $\begin{array}{l}\text { Height } \\
\text { [mAU] }\end{array}$ & $\begin{array}{c}\text { Area } \\
\frac{\circ}{0}\end{array}$ \\
\hline--- & ------ & & & --------- & ------- & ------- \\
\hline 1 & 6.472 & BV & 0.1617 & 84.68909 & 7.80082 & 2.0620 \\
\hline 2 & 6.910 & VB & 0.1662 & 4022.50708 & 374.93903 & 97.9380 \\
\hline
\end{tabular}

Totals:

$4107.19617 \quad 382.73984$ 
methyl 2-(((2-methoxyphenyl)(phenyl)methyl)amino)acetate (2f)
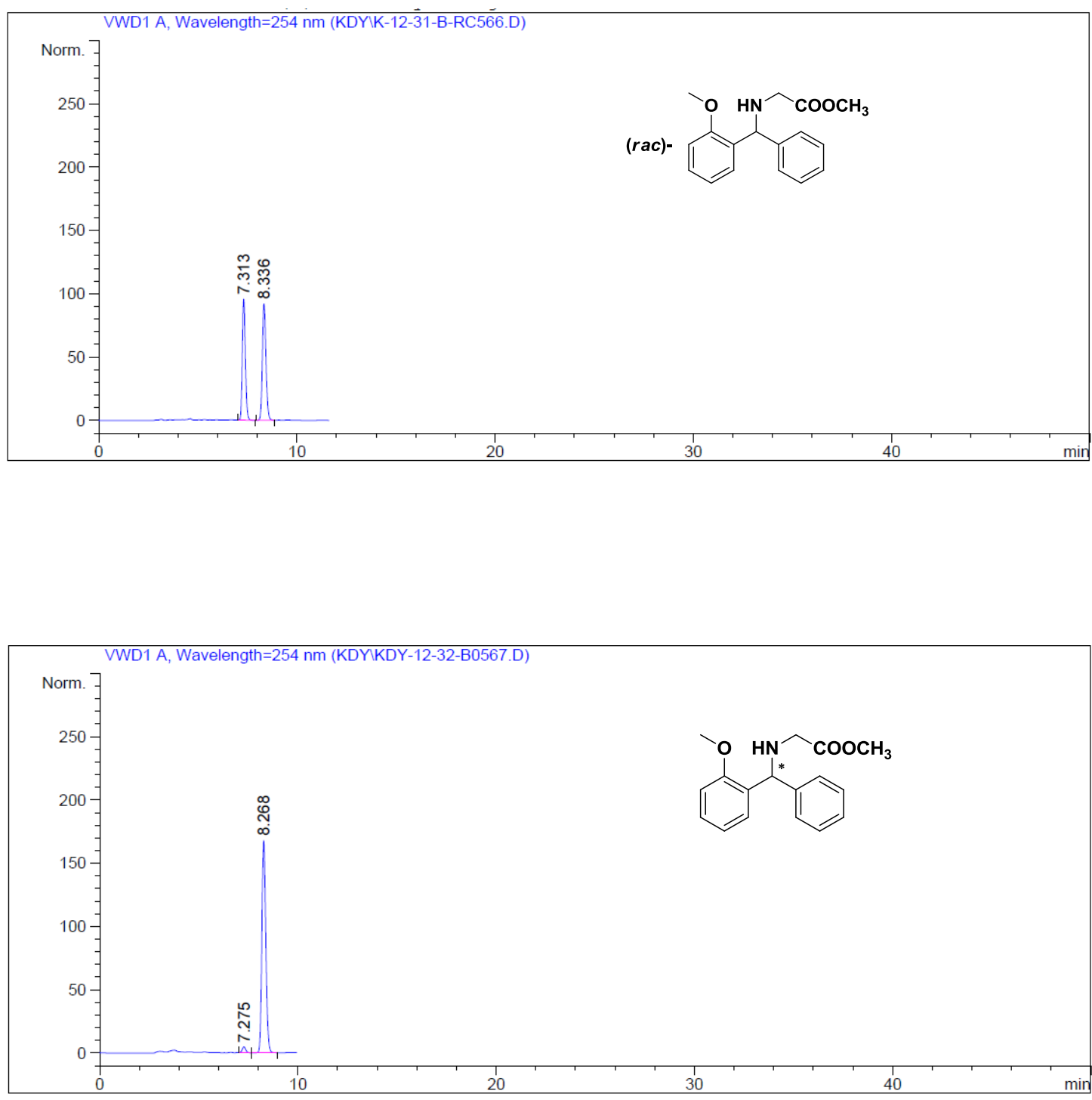

\begin{tabular}{|c|c|c|c|c|c|c|}
\hline $\begin{array}{c}\text { Peak } \\
\#\end{array}$ & $\begin{array}{c}\text { RetTime } \\
\text { [min] }\end{array}$ & Type & $\begin{array}{l}\text { Width } \\
\text { [min] }\end{array}$ & $\begin{array}{c}\text { Area } \\
{\left[\mathrm{mAU}^{*} \mathrm{~S}\right]}\end{array}$ & $\begin{array}{l}\text { Height } \\
{[\mathrm{mAU}]}\end{array}$ & $\begin{array}{c}\text { Area } \\
\quad \circ\end{array}$ \\
\hline 1 & 7.275 & $B$ & $0.1928=$ & 56.71198 & 459962 & 2.3797 \\
\hline 2 & 8.268 & $\mathrm{BB}$ & 0.2159 & 2326.46655 & 167.67757 & 97.6203 \\
\hline ota & $:$ & & & 2383.17853 & 172.27718 & \\
\hline
\end{tabular}


methyl 2-(((2-chlorophenyl)(phenyl)methyl)amino)acetate (2g)
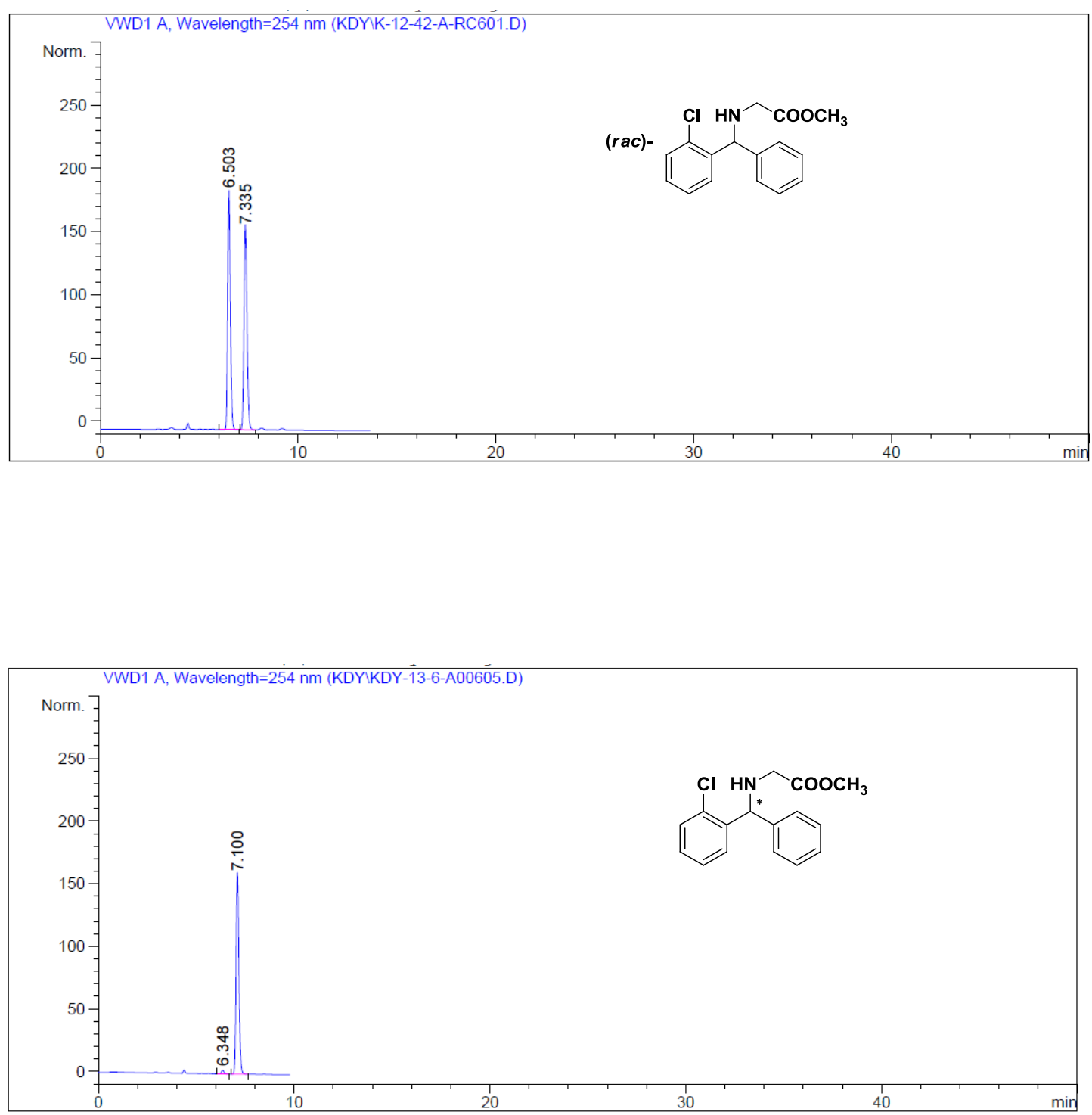

\begin{tabular}{|c|c|c|c|c|c|c|}
\hline $\begin{array}{c}\text { Peak } \\
\text { \# }\end{array}$ & $\begin{array}{c}\text { RetTime } \\
\text { [min] }\end{array}$ & Type & $\begin{array}{l}\text { Width } \\
\text { [min] }\end{array}$ & $\begin{array}{c}\text { Area } \\
{\left[\mathrm{mAU}^{*} \mathrm{~S}\right]}\end{array}$ & $\begin{array}{l}\text { Height } \\
{[\mathrm{mAU}]}\end{array}$ & $\begin{array}{c}\text { Area } \\
\frac{\circ}{0}\end{array}$ \\
\hline-- & - & & ------ & |---------- & -------- & ------1 \\
\hline 1 & 6.348 & BB & 0.1556 & 27.41905 & 2.97426 & 1.6593 \\
\hline 2 & 7.100 & BB & 0.1675 & 1625.01099 & 158.59970 & 98.3407 \\
\hline otal & . & & & 1652.43004 & 161.57396 & \\
\hline
\end{tabular}


methyl 2-(((2-chlorophenyl)(3-methoxyphenyl)methyl)amino)acetate (2h)
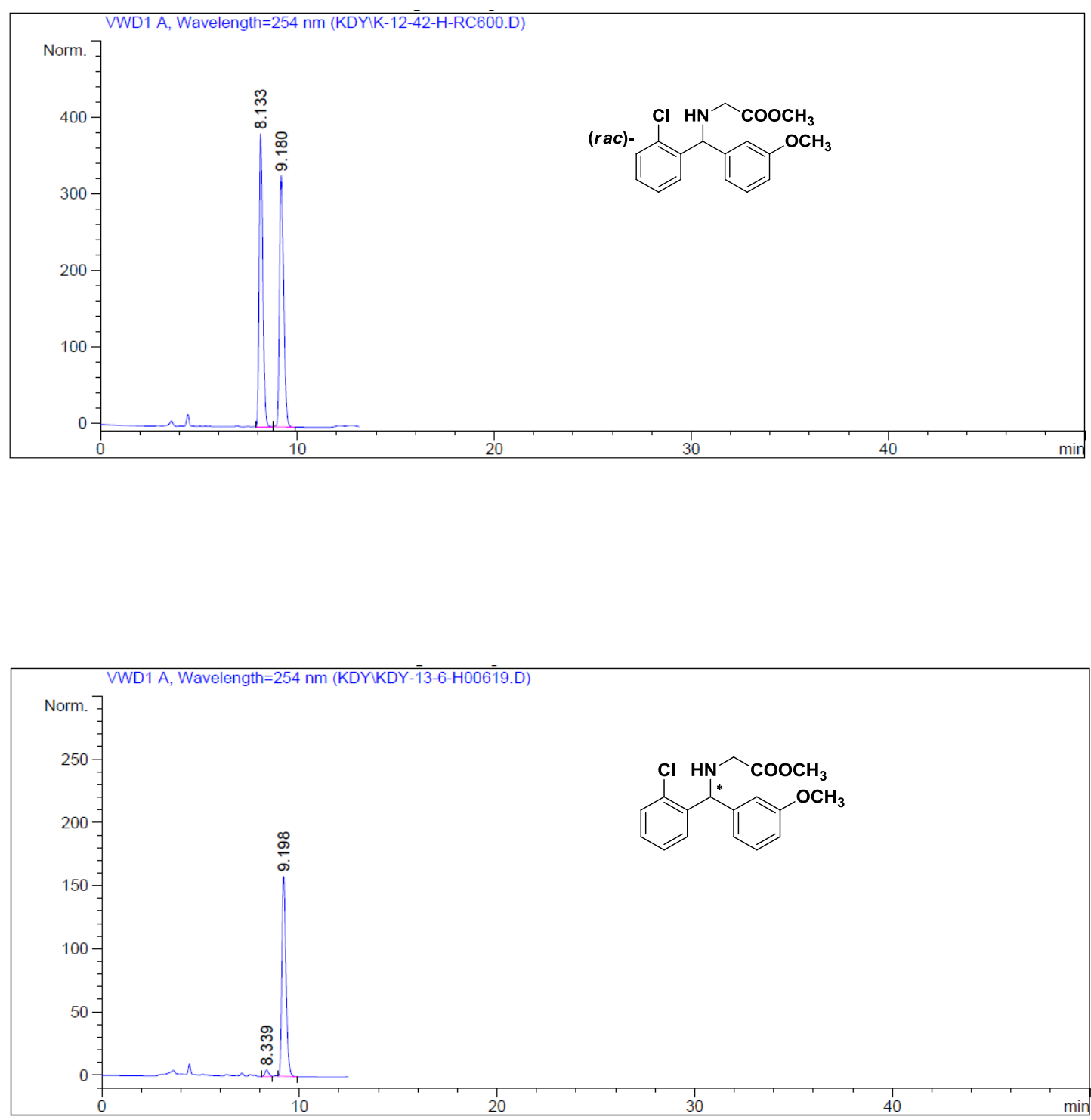

\begin{tabular}{|c|c|c|c|c|c|c|}
\hline $\begin{array}{c}\text { Peak } \\
\quad \#\end{array}$ & $\begin{array}{c}\text { RetTime } \\
\text { [min] }\end{array}$ & Type & $\begin{array}{l}\text { Width } \\
{[\mathrm{min}]}\end{array}$ & $\begin{array}{c}\text { Area } \\
{\left[\mathrm{mAU}^{*} \mathrm{~s}\right]}\end{array}$ & $\begin{array}{l}\text { Height } \\
{[\mathrm{mAU}]}\end{array}$ & $\begin{array}{c}\text { Area } \\
\quad \circ\end{array}$ \\
\hline & & & ----- & $\mid---------$ & ------- & \\
\hline 1 & 8.339 & BB & 0.1977 & 64.03210 & 4.99031 & 2.7406 \\
\hline 2 & 9.198 & BB & 0.2207 & 2272.35864 & 158.12172 & 97.2594 \\
\hline tal & : & & & 2336.39074 & 163.11203 & \\
\hline
\end{tabular}


methyl 2-(((2-chlorophenyl)(m-tolyl)methyl)amino)acetate (2i)
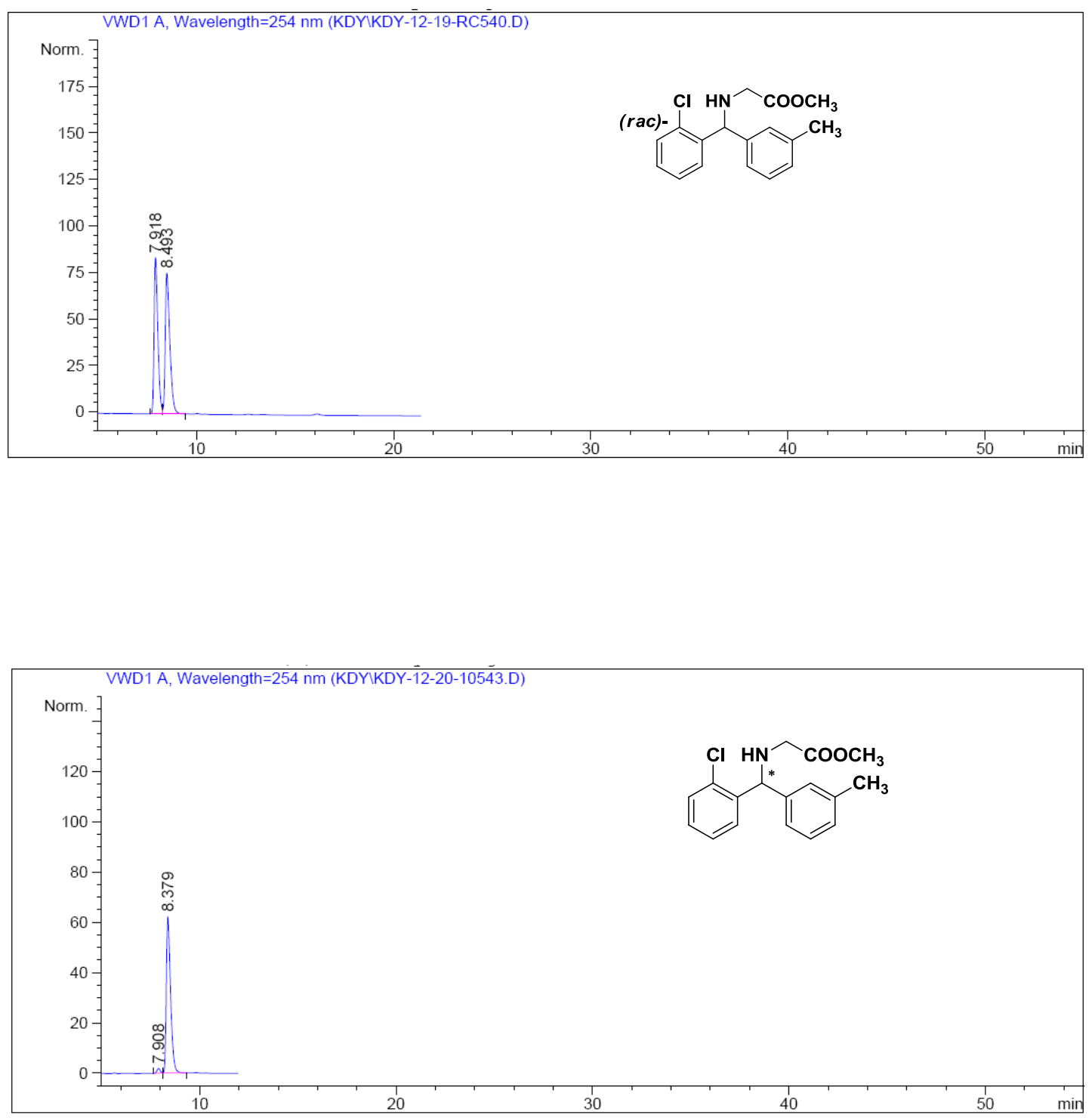

\begin{tabular}{|c|c|c|c|c|c|c|}
\hline $\begin{array}{c}\text { Peak } \\
\text { \# }\end{array}$ & $\begin{array}{c}\text { RetTime } \\
\text { [min] }\end{array}$ & Type & $\begin{array}{c}\text { Width } \\
\text { [min] }\end{array}$ & $\begin{array}{c}\text { Area } \\
{\left[\mathrm{mAU}^{*} \mathrm{~S}\right]}\end{array}$ & $\begin{array}{l}\text { Height } \\
{[\mathrm{mAU}]}\end{array}$ & $\begin{array}{c}\text { Area } \\
\quad \frac{\circ}{b}\end{array}$ \\
\hline & & & & - & -2 & \\
\hline 1 & 7.908 & $\mathrm{BB}$ & 0.1759 & 20.76943 & 1.85048 & 2.1670 \\
\hline 2 & 8.379 & BB & 0.2247 & 937.65991 & 62.25066 & 97.8330 \\
\hline ota & & & & 958.42934 & 64.10114 & \\
\hline
\end{tabular}


methyl 2-(((2-chlorophenyl)(p-tolyl)methyl)amino)acetate $(2 \mathbf{j})$
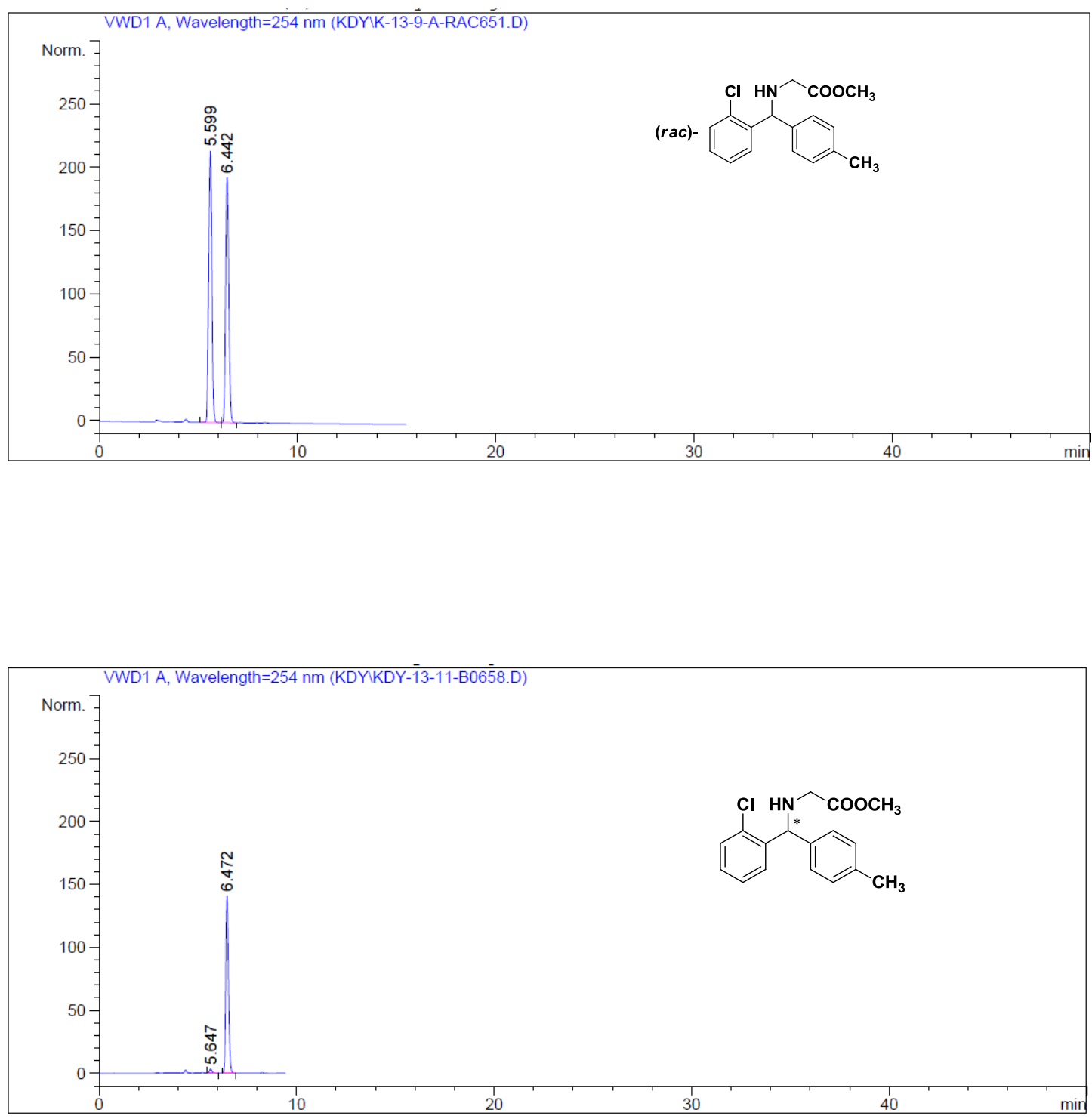

\begin{tabular}{|c|c|c|c|c|c|c|}
\hline $\begin{array}{c}\text { Peak } \\
\quad \#\end{array}$ & $\begin{array}{c}\text { RetTime } \\
\text { [min] }\end{array}$ & Type & $\begin{array}{l}\text { Width } \\
\text { [min] }\end{array}$ & $\begin{array}{c}\text { Area } \\
{\left[\mathrm{mAU}^{*} \mathrm{~s}\right]}\end{array}$ & $\begin{array}{l}\text { Height } \\
{[\mathrm{mAU}]}\end{array}$ & $\begin{array}{c}\text { Area } \\
\text { \% }\end{array}$ \\
\hline---- & ------- & & ------- & | ---------- & ---------- & -------- \\
\hline 1 & 5.647 & $\mathrm{BB}$ & 0.1256 & 25.27577 & 3.11680 & 1.8441 \\
\hline 2 & 6.472 & $\mathrm{BB}$ & 0.1474 & 1345.34204 & 140.86060 & 98.1559 \\
\hline Total & ls: & & & 1370.61781 & 7740 & \\
\hline
\end{tabular}


methyl 2-(((2-chlorophenyl)(4-fluorophenyl)methyl)amino)acetate (2k)
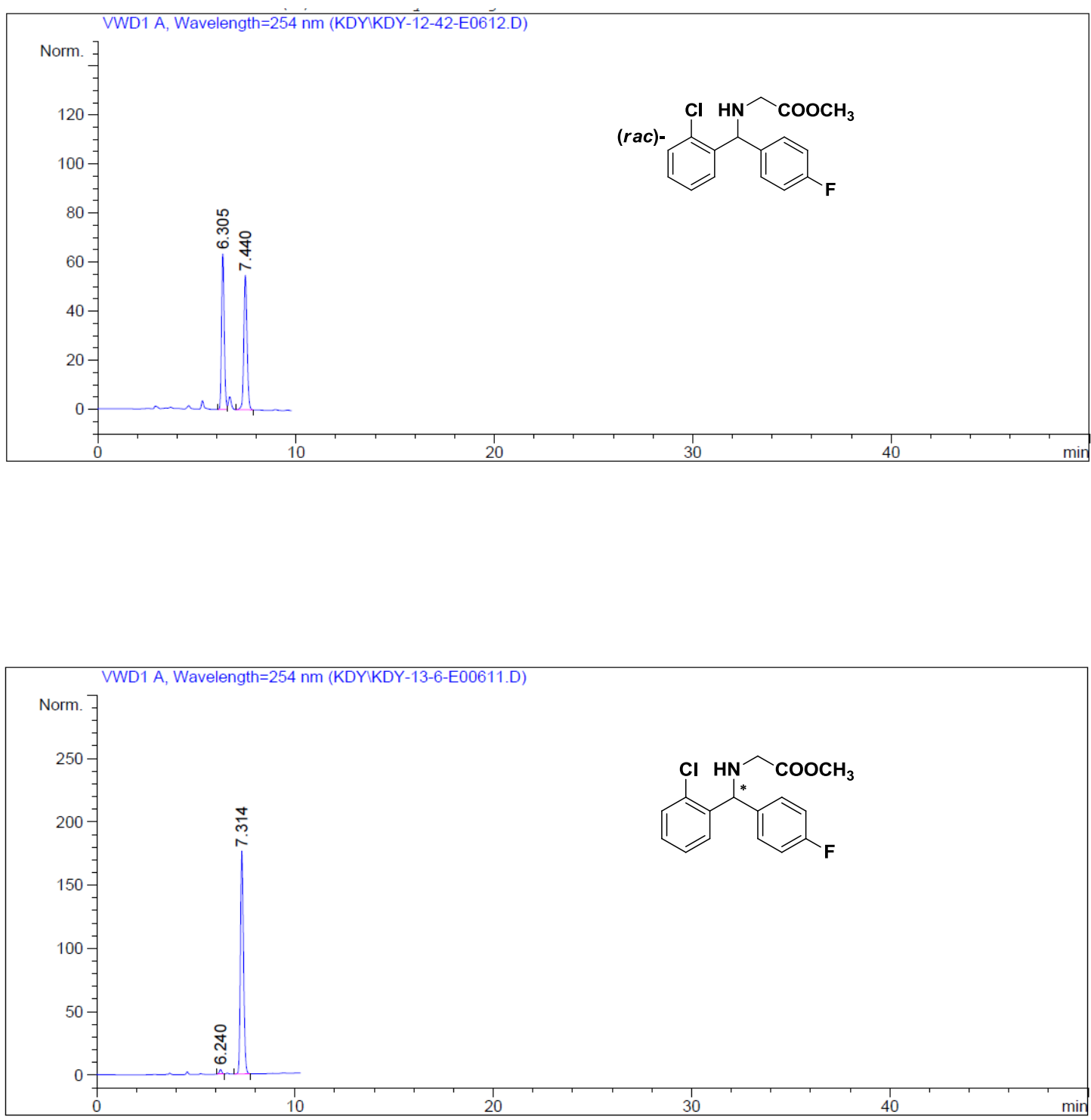

\begin{tabular}{|c|c|c|c|c|c|c|}
\hline $\begin{array}{c}\text { Peak } \\
\#\end{array}$ & $\begin{array}{c}\text { RetTime } \\
\text { [min] }\end{array}$ & Type & $\begin{array}{c}\text { Width } \\
\text { [min] }\end{array}$ & $\begin{array}{c}\text { Area } \\
{\left[\mathrm{mAU}^{*} \mathrm{~S}\right]}\end{array}$ & $\begin{array}{l}\text { Height } \\
\text { [mAU] }\end{array}$ & $\begin{array}{c}\text { Area } \\
\quad \frac{\circ}{0}\end{array}$ \\
\hline 1 & 6.240 & $\mathrm{BB}$ & 0.1329 & 30.57846 & 3.57080 & 1.5813 \\
\hline 2 & 7.314 & $\mathrm{BB}$ & 0.1662 & 1903.18457 & 175.99194 & 98.4187 \\
\hline Total & $\mathrm{s}$ & & & 1933.76303 & 179.56275 & \\
\hline
\end{tabular}


methyl 2-(((2-chlorophenyl)(4-chlorophenyl)methyl)amino)acetate (2l)
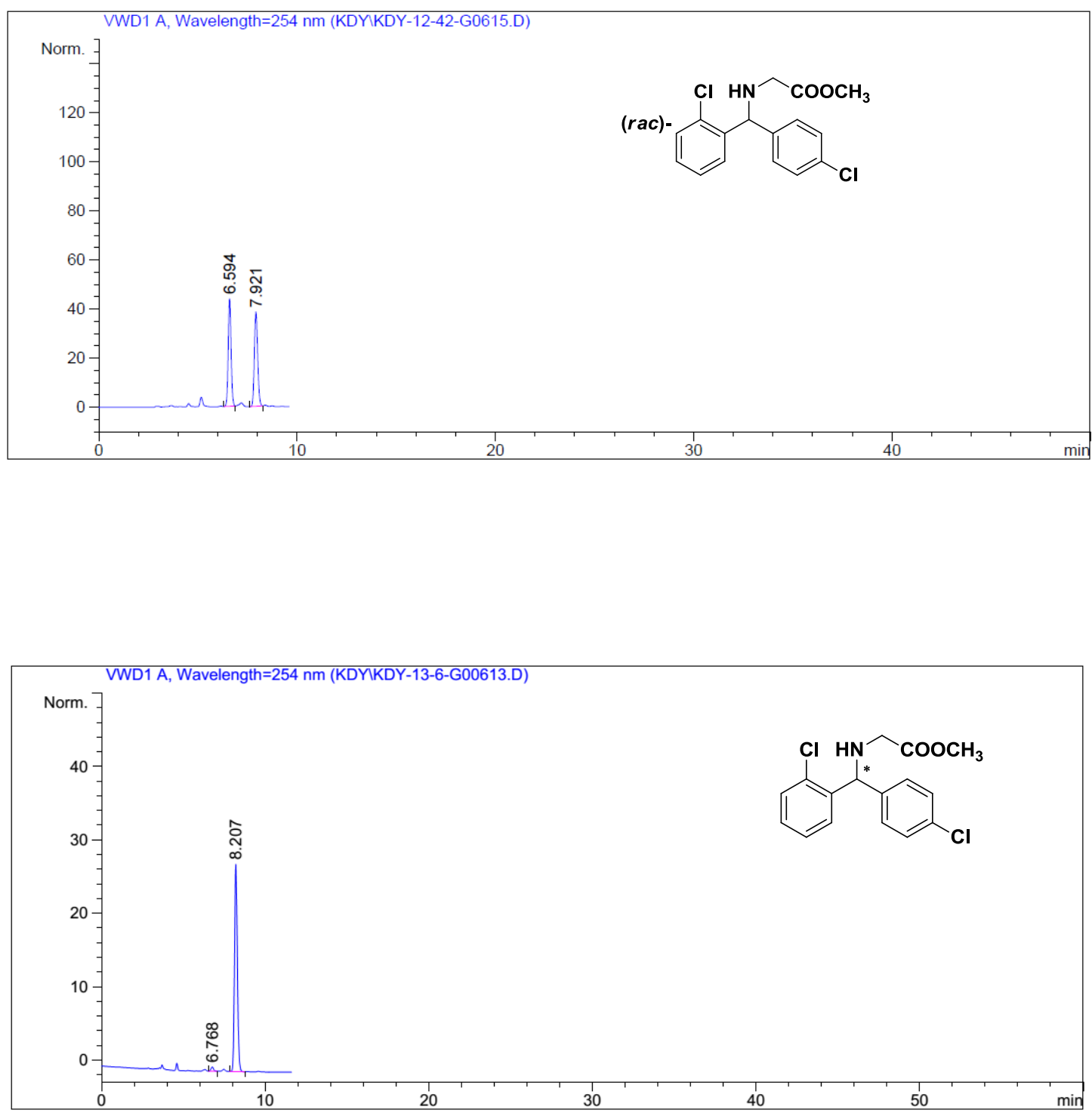

\begin{tabular}{|c|c|c|c|c|c|c|}
\hline $\begin{array}{c}\text { Peak } \\
\quad \#\end{array}$ & $\begin{array}{c}\text { RetTime } \\
\text { [min] }\end{array}$ & Type & $\begin{array}{c}\text { Width } \\
\text { [min] }\end{array}$ & $\begin{array}{c}\text { Area } \\
{\left[\mathrm{mAU}^{\star} \mathrm{s}\right]}\end{array}$ & $\begin{array}{l}\text { Height } \\
\text { [mAU] }\end{array}$ & $\begin{array}{c}\text { Area } \\
\text { 응 }\end{array}$ \\
\hline---1 & ------- & & ------- & --------- & --------- & $-------\mid$ \\
\hline 1 & 6.768 & BB & 0.1657 & 6.10054 & $5.61836 e-1$ & 1.7644 \\
\hline 2 & 8.207 & BB & 0.1853 & 339.64920 & 28.24668 & 98.2356 \\
\hline tal & & & & 345.74974 & 852 & \\
\hline
\end{tabular}


methyl 2-(((2-bromophenyl)(phenyl)methyl)amino)acetate (2m)
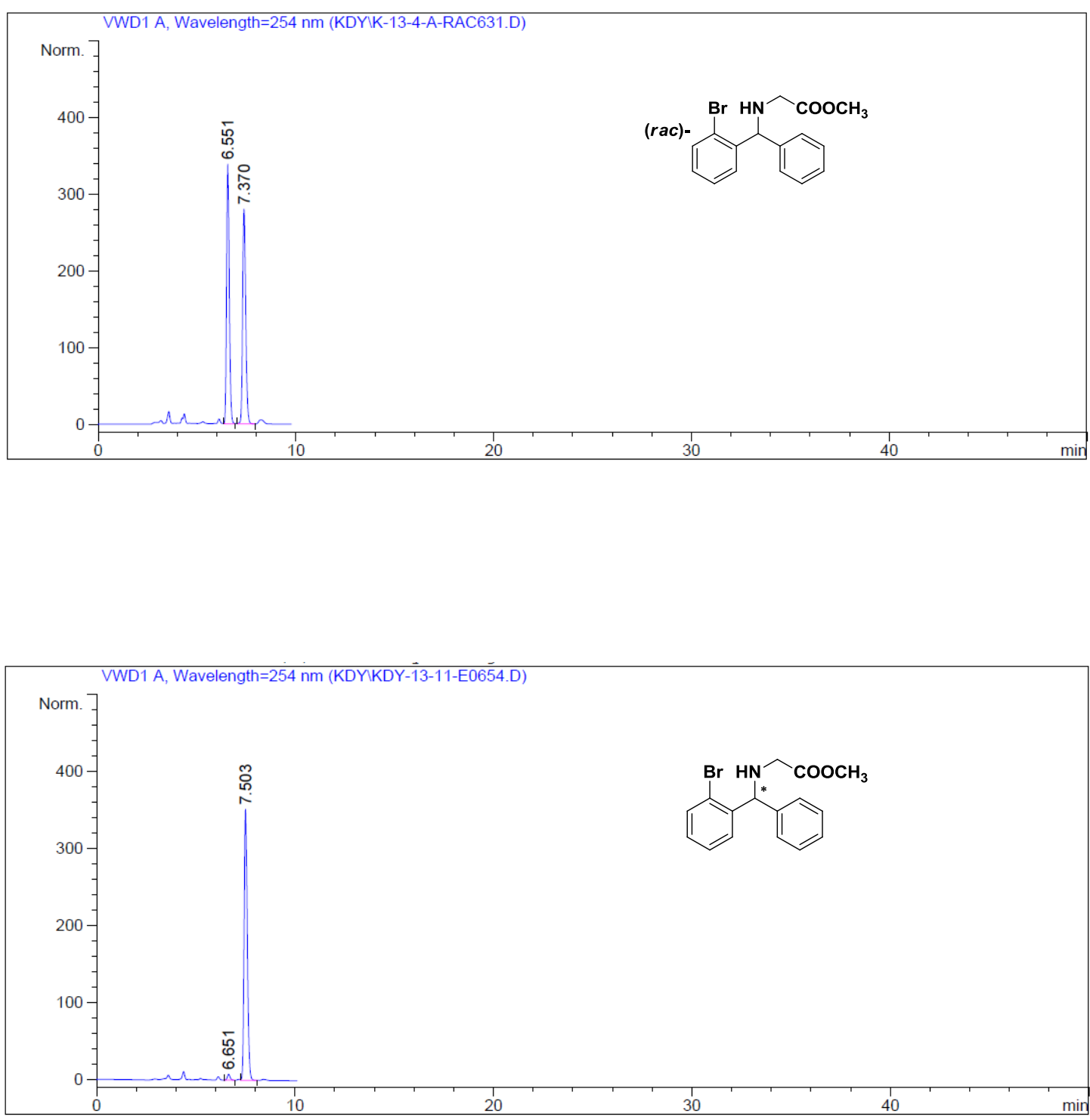

\begin{tabular}{|c|c|c|c|c|c|c|}
\hline $\begin{array}{c}\text { Peak } \\
\quad \#\end{array}$ & $\begin{array}{c}\text { RetTime } \\
\text { [min] }\end{array}$ & Type & $\begin{array}{l}\text { Width } \\
\text { [min] }\end{array}$ & $\begin{array}{c}\text { Area } \\
{\left[\mathrm{mAU}^{*} \mathrm{~s}\right]}\end{array}$ & $\begin{array}{l}\text { Height } \\
\text { [mAU] }\end{array}$ & $\begin{array}{c}\text { Area } \\
\text { 응 }\end{array}$ \\
\hline 1 & 6.651 & BB & 0.1498 & 74.82027 & 7.66963 & 1.8584 \\
\hline 2 & 7.503 & VB & 0.1740 & 3951.24341 & 351.93756 & 98.1416 \\
\hline Total & : & & & 4026.06367 & 359.60719 & \\
\hline
\end{tabular}


methyl 2-(((2-bromophenyl)(3-fluorophenyl)methyl)amino)acetate (2n)
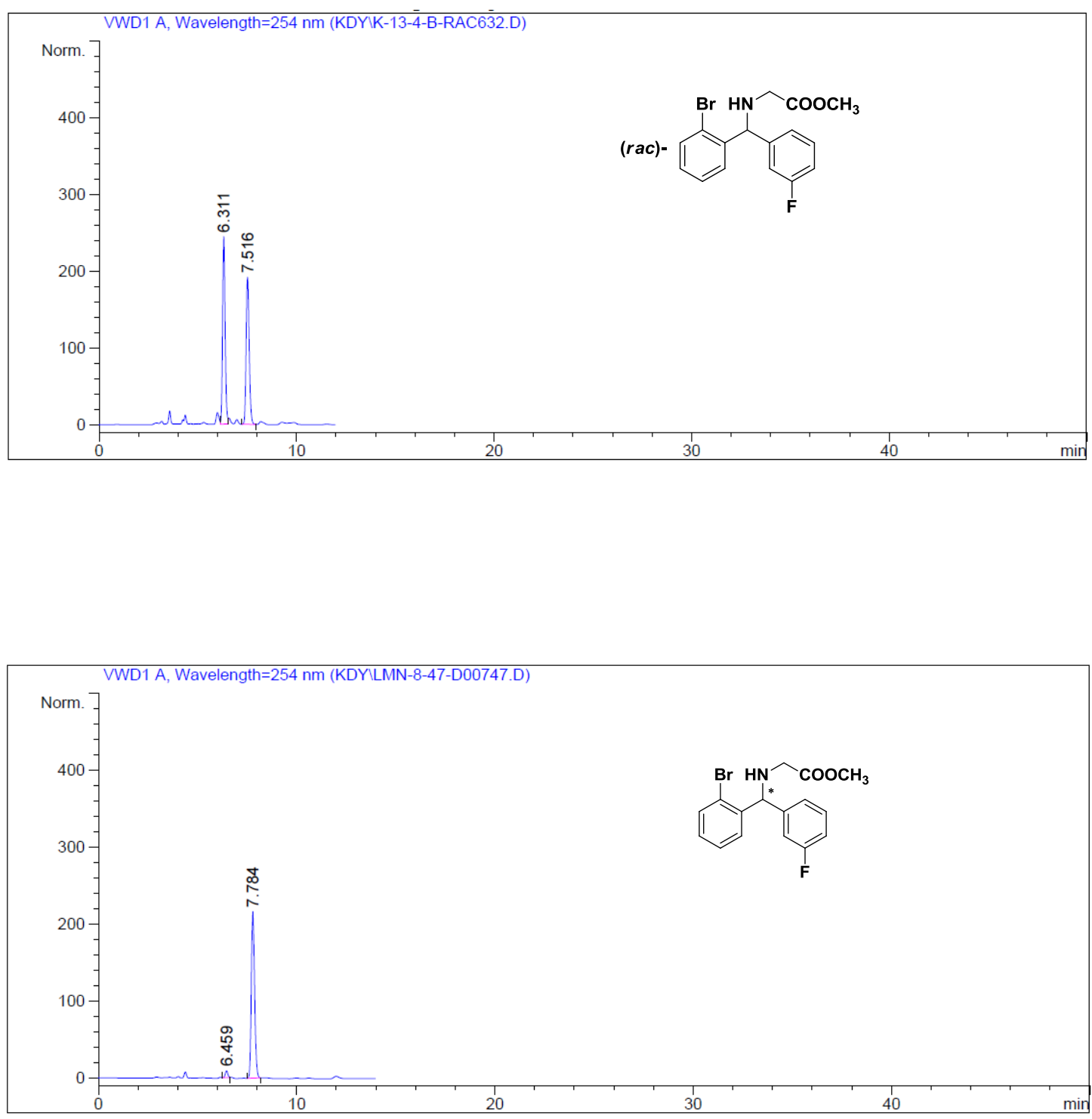

\begin{tabular}{|c|c|c|c|c|c|c|}
\hline $\begin{array}{c}\text { Peak } \\
\#\end{array}$ & $\begin{array}{c}\text { RetTime } \\
\text { [min] }\end{array}$ & Type & $\begin{array}{c}\text { Width } \\
{[\mathrm{min}]}\end{array}$ & $\begin{array}{c}\text { Area } \\
{\left[\mathrm{mAU}^{*} \mathrm{~s}\right]}\end{array}$ & $\begin{array}{l}\text { Height } \\
\text { [mAU] }\end{array}$ & $\begin{array}{c}\text { Area } \\
\frac{\circ}{0}\end{array}$ \\
\hline--- & 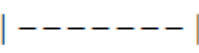 & & ------ & $\mid----------$ & --------- & $-------\mid$ \\
\hline 1 & 6.459 & $\mathrm{BB}$ & 0.1386 & 76.43546 & 8.69483 & 2.9730 \\
\hline 2 & 7.784 & BB & 0.1784 & 2494.50952 & 216.54170 & 97.0270 \\
\hline Total & $1 \mathrm{~s}:$ & & & 2570.94498 & 225.23653 & \\
\hline
\end{tabular}


methyl 2-(((2-bromophenyl)(4-fluorophenyl)methyl)amino)acetate (2o)
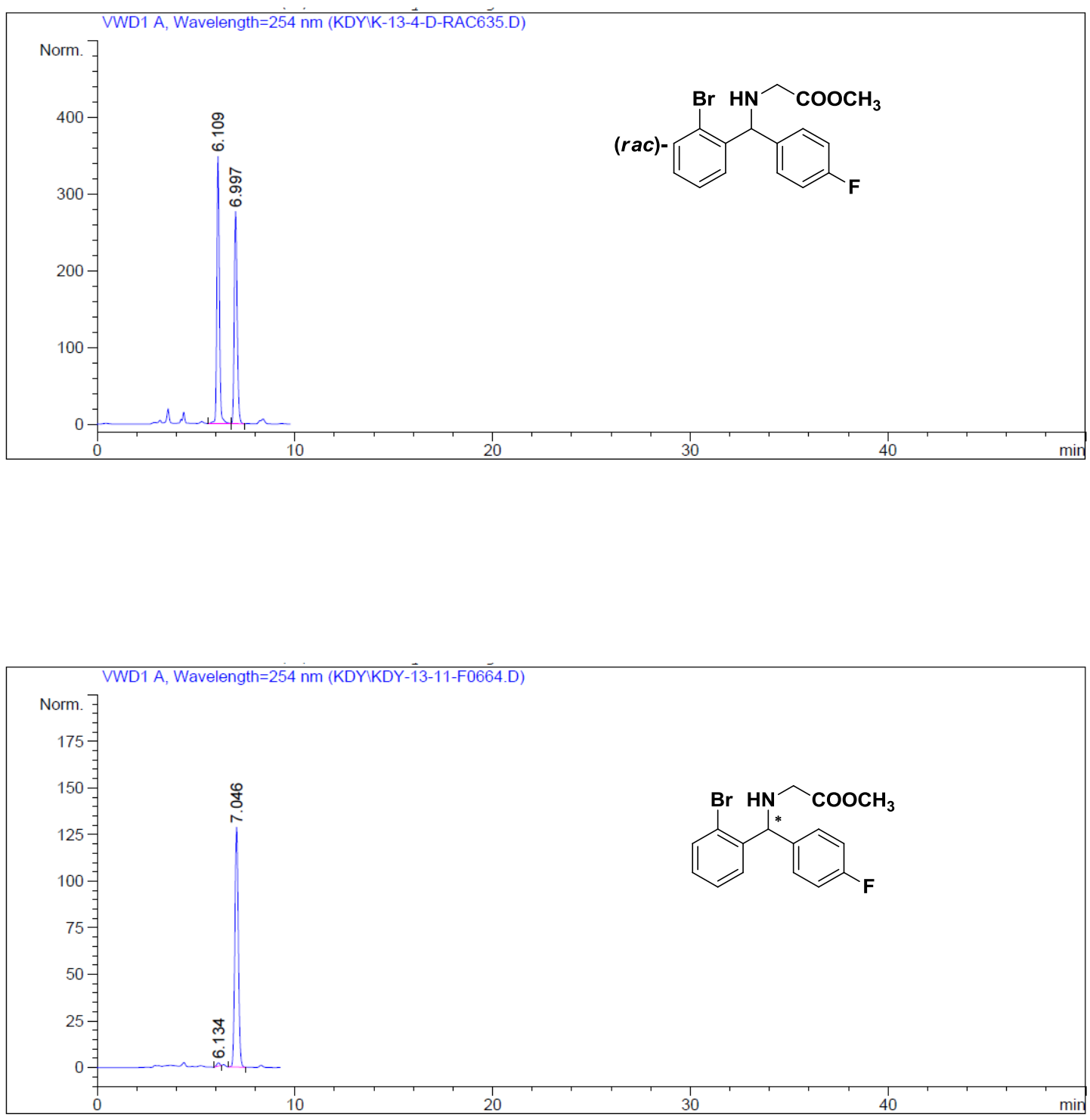

\begin{tabular}{|c|c|c|c|c|c|c|}
\hline $\begin{array}{c}\text { Peak } \\
\quad \#\end{array}$ & $\begin{array}{c}\text { RetTime } \\
\text { [min] }\end{array}$ & Type & $\begin{array}{c}\text { Width } \\
{[\mathrm{min}]}\end{array}$ & $\begin{array}{c}\text { Area } \\
{\left[\mathrm{mAU}^{*} \mathrm{~S}\right]}\end{array}$ & $\begin{array}{l}\text { Height } \\
{[\mathrm{mAU}]}\end{array}$ & $\begin{array}{c}\text { Area } \\
\frac{\circ}{0}\end{array}$ \\
\hline$=-$ & ---- & & ------ & ----------- & --------- & ------ \\
\hline 1 & 6.134 & BB & 0.1785 & 18.95439 & 1.77652 & 1.1941 \\
\hline 2 & 7.046 & $\mathrm{BB}$ & 0.1892 & 1568.41321 & 128.64769 & 98.8059 \\
\hline
\end{tabular}

Totals :

$1587.36760 \quad 130.42421$ 
methyl 2-(((2-bromophenyl)(4-chlorophenyl)methyl)amino)acetate (2p)
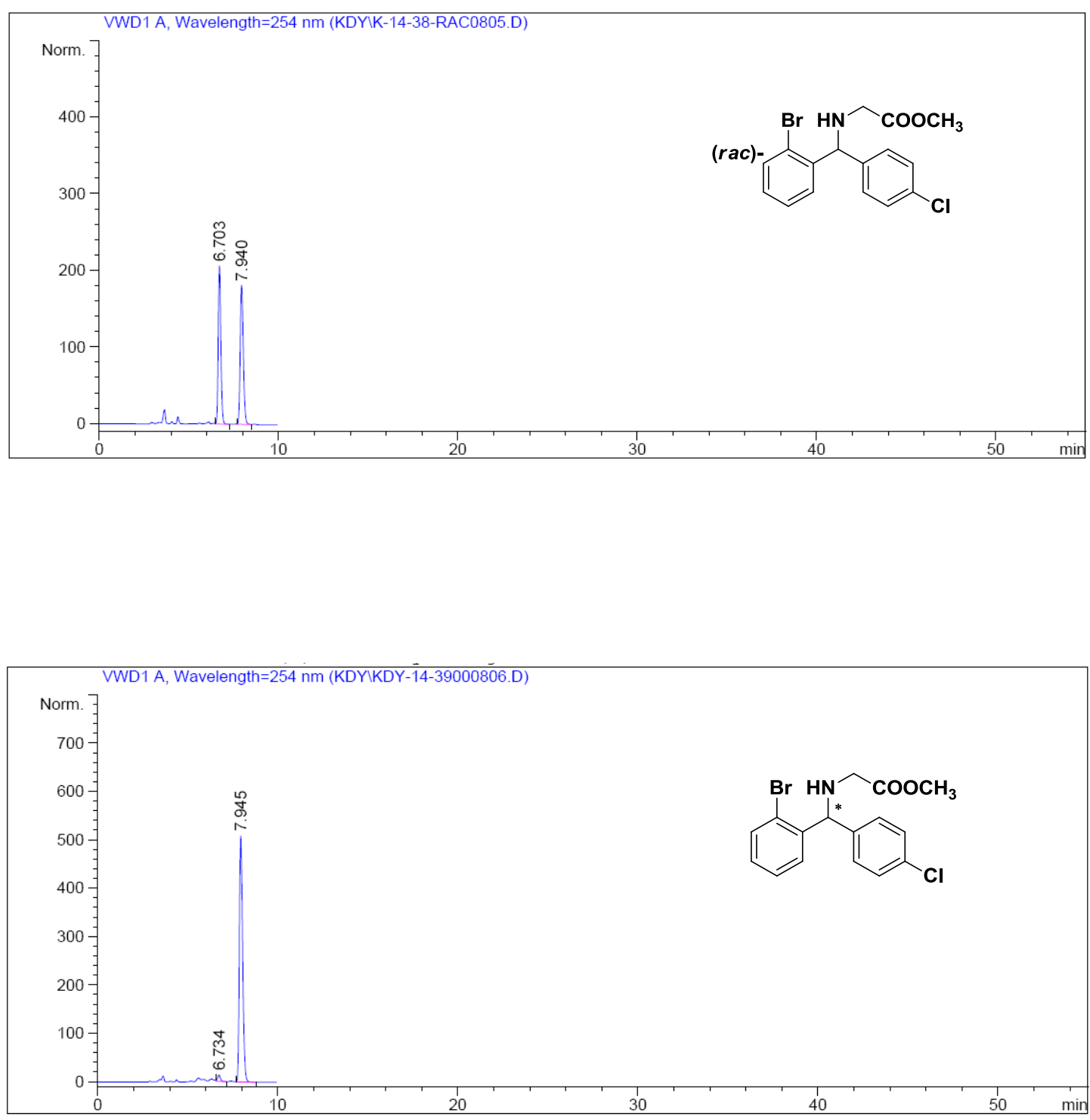

\begin{tabular}{|c|c|c|c|c|c|c|}
\hline $\begin{array}{c}\text { Peak } \\
\#\end{array}$ & $\begin{array}{c}\text { RetTime } \\
\text { [min] }\end{array}$ & Type & $\begin{array}{c}\text { Width } \\
\text { [min] }\end{array}$ & $\begin{array}{c}\text { Area } \\
{\left[\mathrm{mAU}^{*} \mathrm{~S}\right]}\end{array}$ & $\begin{array}{l}\text { Height } \\
{[\mathrm{mAU}]}\end{array}$ & $\begin{array}{c}\text { Area } \\
\frac{\circ}{0}\end{array}$ \\
\hline-- & ------ & 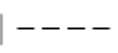 & ------- & ---------- & ---------- & ------ \\
\hline 1 & 6.734 & VB & 0.1624 & 141.56326 & 13.17512 & 2.1405 \\
\hline 2 & 7.945 & VB & 0.1975 & 6472.01514 & 508.42920 & 97.8595 \\
\hline otal & L $:$ & & & 6613.57840 & 521.60432 & \\
\hline
\end{tabular}


methyl 2-(((2-fluorophenyl)(phenyl)methyl)amino)acetate (2q)
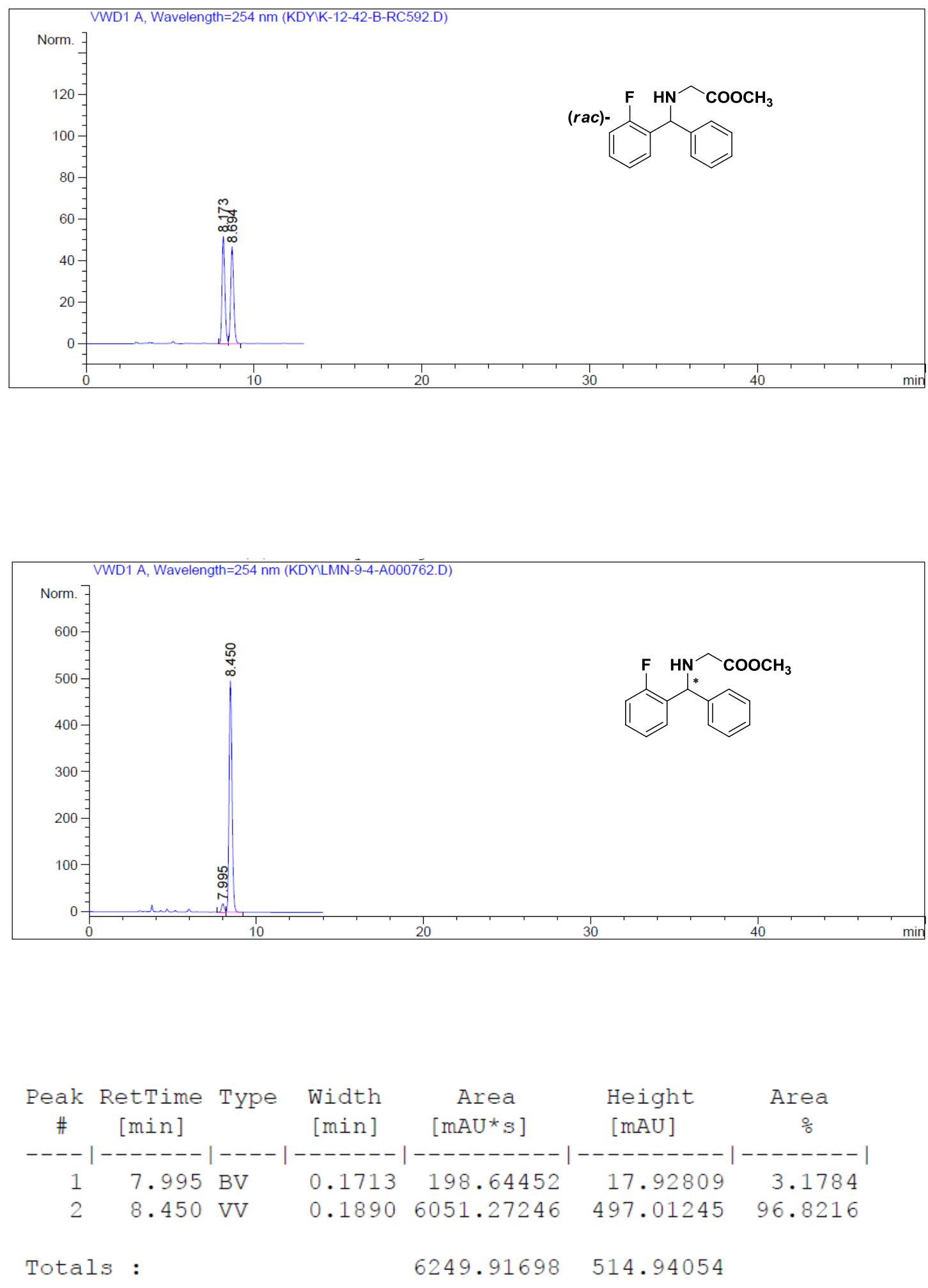

S74 
methyl 2-(((2-fluorophenyl)(m-tolyl)methyl)amino)acetate (2r)
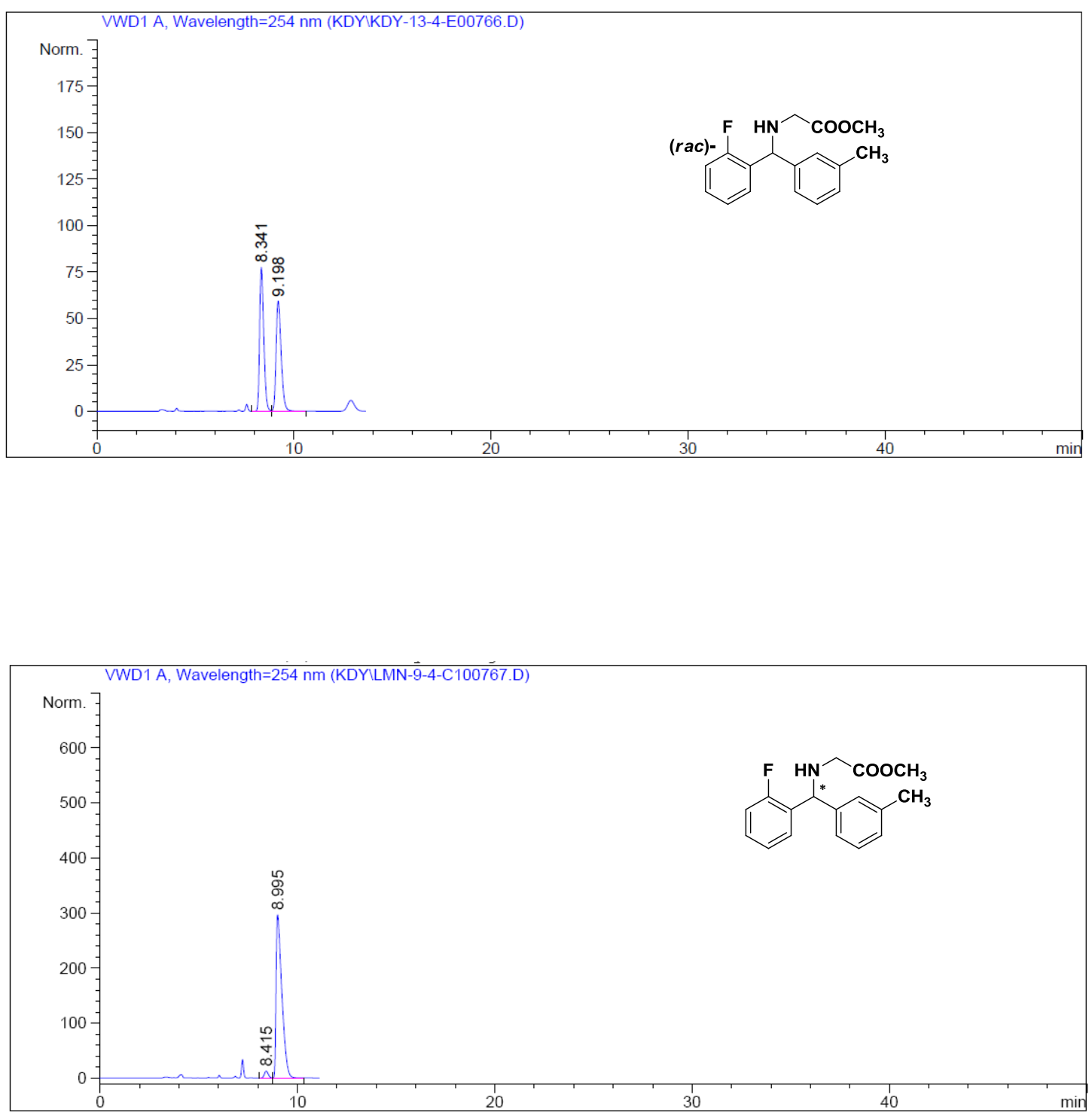

\begin{tabular}{|c|c|c|c|c|c|c|}
\hline $\begin{array}{c}\text { Peak } \\
\#\end{array}$ & $\begin{array}{c}\text { RetTime } \\
\text { [min] }\end{array}$ & Type & $\begin{array}{l}\text { Width } \\
\text { [min] }\end{array}$ & $\begin{array}{c}\text { Area } \\
{\left[\mathrm{mAU}^{*} \mathrm{~S}\right]}\end{array}$ & $\begin{array}{l}\text { Height } \\
\text { [mAU] }\end{array}$ & $\begin{array}{c}\text { Area } \\
\quad \frac{\circ}{0}\end{array}$ \\
\hline--1 & 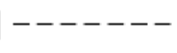 & & ------ & ---------- & --------- & ------- \\
\hline 1 & 8.415 & BV & 0.2178 & 178.59250 & 12.71837 & 2.8680 \\
\hline 2 & 8.995 & VV & 0.3000 & 6048.48584 & 296.43839 & 97.1320 \\
\hline Total & $s:$ & & & 6227.07834 & 309.15676 & \\
\hline
\end{tabular}


methyl 2-(((2-fluorophenyl)(4-fluorophenyl)methyl)amino)acetate (2s)
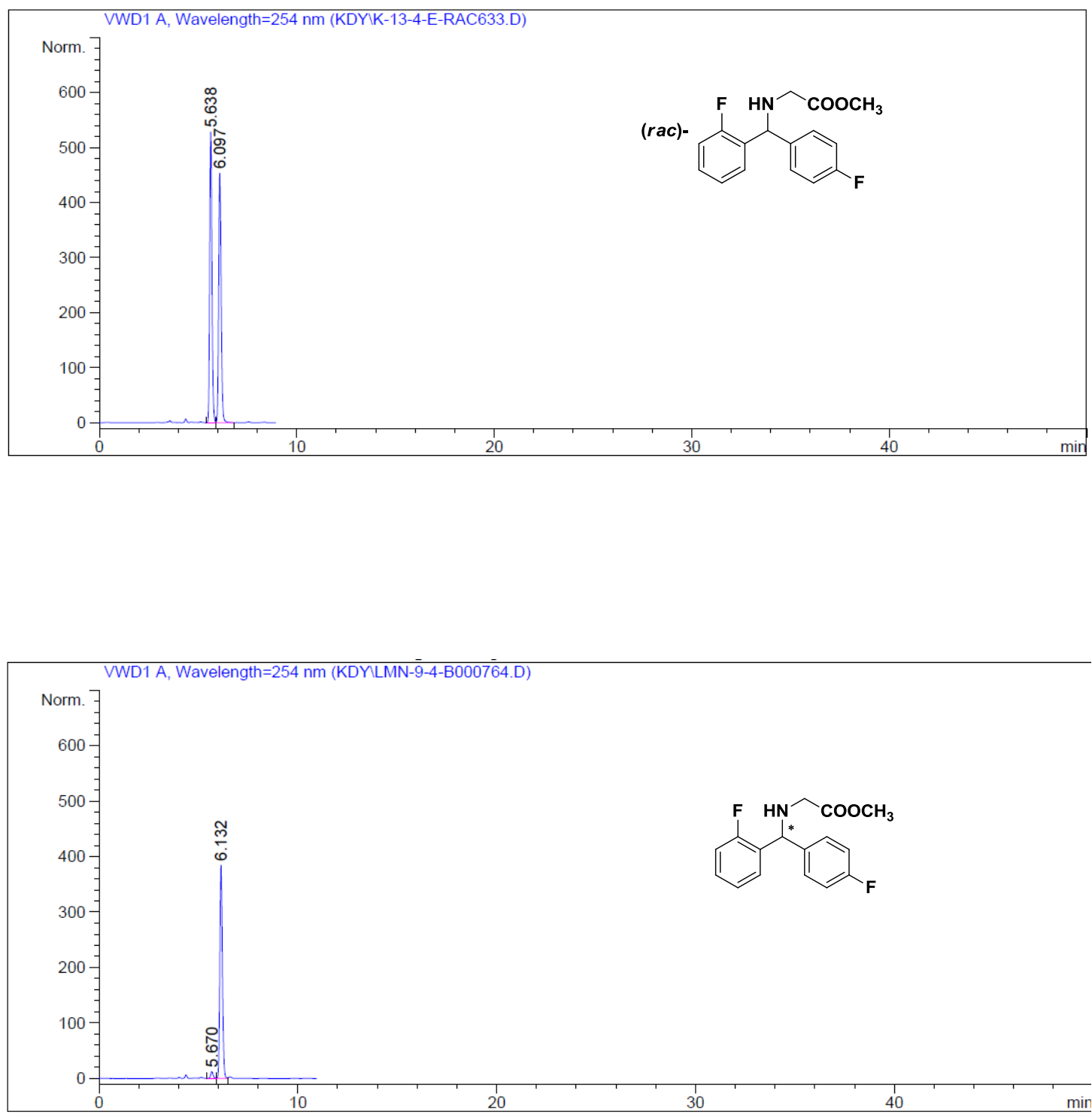

\begin{tabular}{|c|c|c|c|c|c|c|}
\hline $\begin{array}{c}\text { Peak } \\
\quad \#\end{array}$ & $\begin{array}{c}\text { RetTime } \\
\text { [min] }\end{array}$ & Type & $\begin{array}{c}\text { Width } \\
{[\mathrm{min}]}\end{array}$ & $\begin{array}{c}\text { Area } \\
{\left[\mathrm{mAU}^{*} \mathrm{~S}\right]}\end{array}$ & $\begin{array}{l}\text { Height } \\
\text { [mAU] }\end{array}$ & $\begin{array}{c}\text { Area } \\
\text { 응 }\end{array}$ \\
\hline & & & & $\mid----------$ & ------- & ----- \\
\hline 1 & 5.670 & BV & 0.1298 & 102.15746 & 11.94427 & 2.8824 \\
\hline 2 & 6.132 & VV & 0.1385 & 3442.07397 & 384.32925 & 97.1176 \\
\hline ota & & & & 3544.23144 & 396.27352 & \\
\hline
\end{tabular}


methyl 2-((naphthalen-1-yl(phenyl)methyl)amino)acetatev (2t)
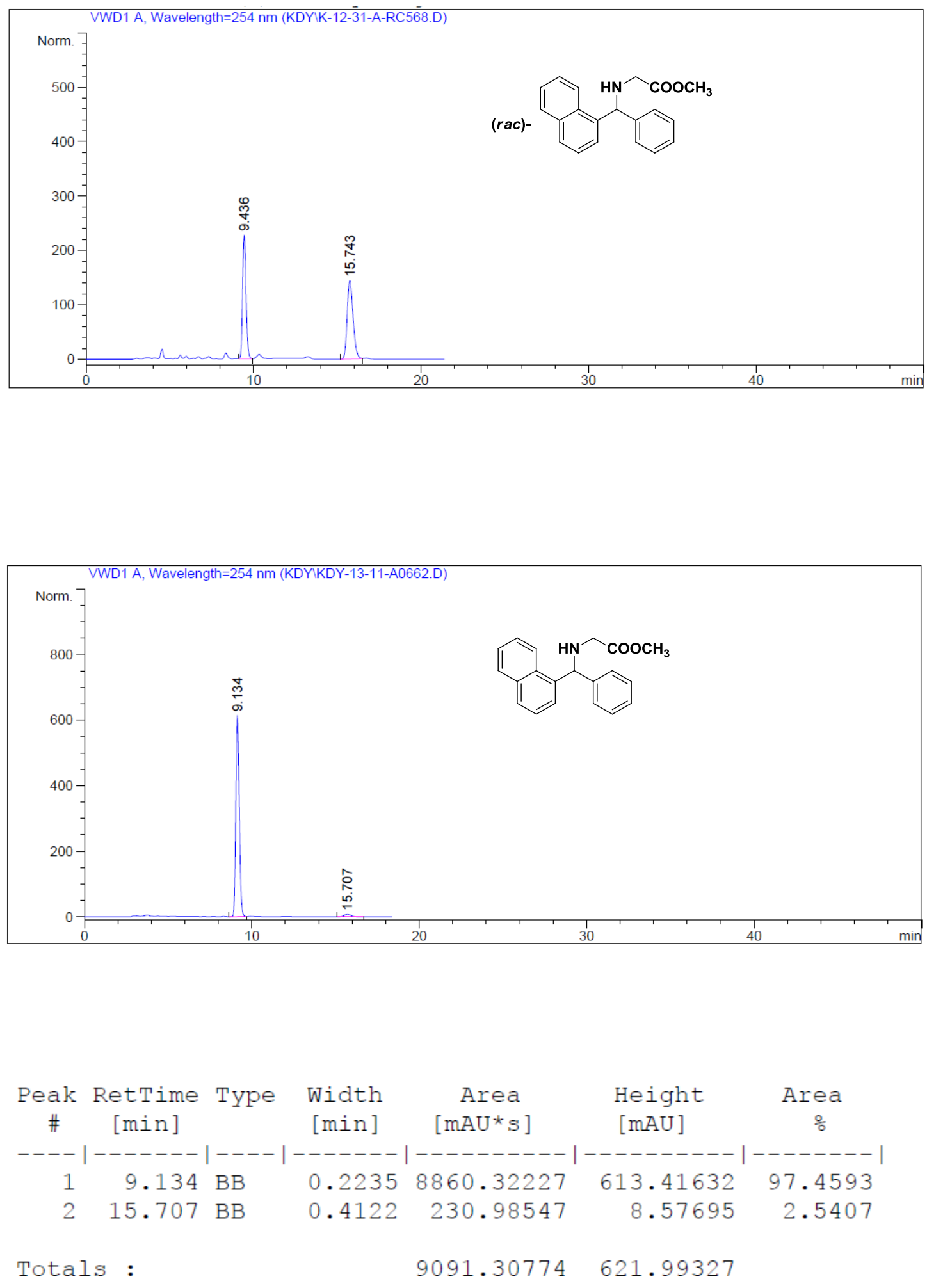

S77 
ethyl 2-((phenyl(o-tolyl)methyl)amino)acetate (2u)
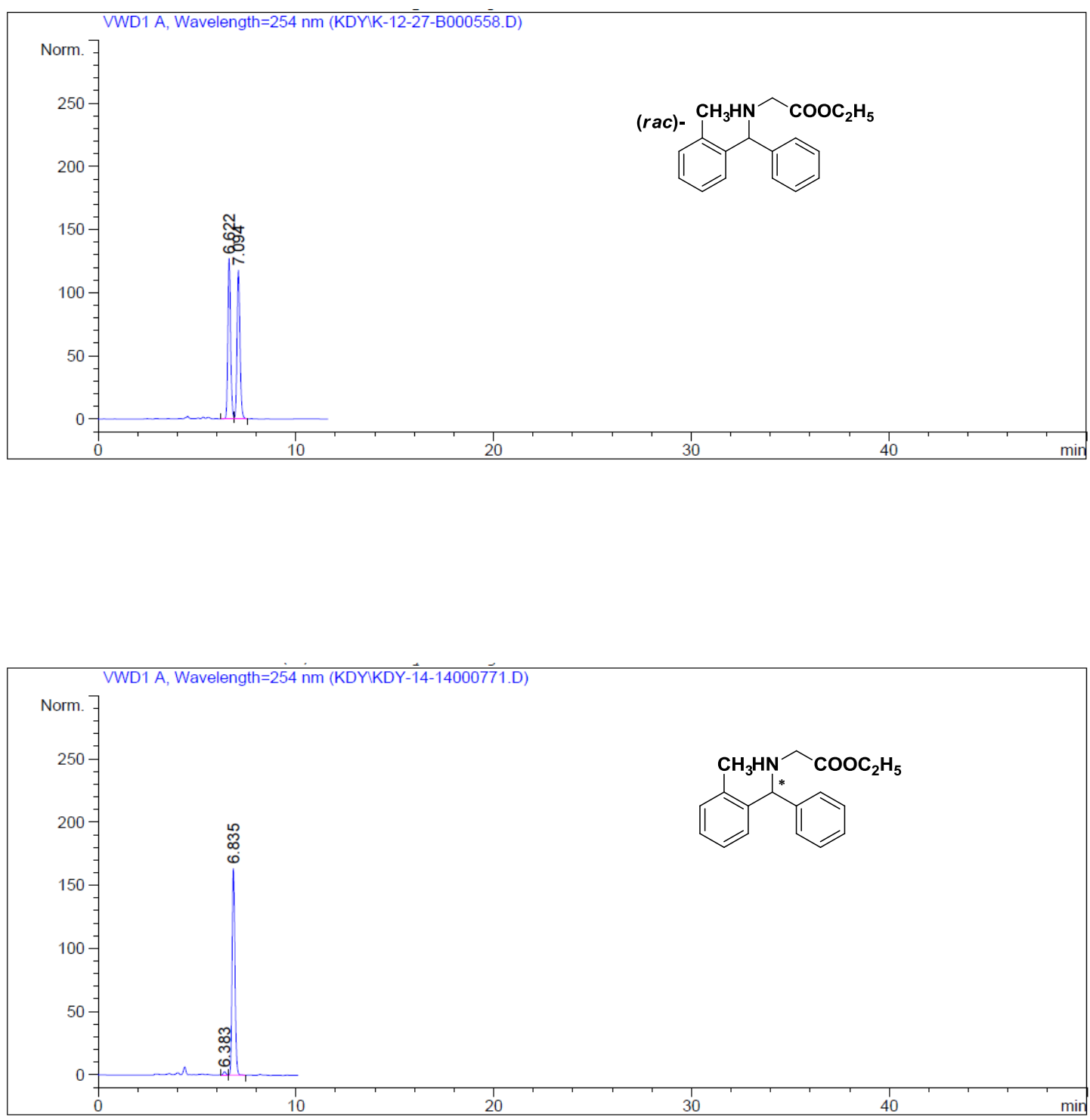

\begin{tabular}{|c|c|c|c|c|c|c|}
\hline $\begin{array}{c}\text { Peak } \\
\quad \#\end{array}$ & $\begin{array}{c}\text { RetTime } \\
\text { [min] }\end{array}$ & Type & $\begin{array}{c}\text { Width } \\
\text { [min] }\end{array}$ & $\begin{array}{c}\text { Area } \\
{\left[\mathrm{mAU}^{*} \mathrm{~S}\right]}\end{array}$ & $\begin{array}{l}\text { Height } \\
\text { [mAU] }\end{array}$ & $\begin{array}{c}\text { Area } \\
\text { 응 }\end{array}$ \\
\hline 1 & 6.383 & $\mathrm{BB}$ & 0.1402 & 22.65653 & 2.53791 & 1.3631 \\
\hline 2 & 6.835 & $\mathrm{BB}$ & 0.1550 & 1639.48779 & 163.44930 & 98.6369 \\
\hline Tota: & Is : & & & 1662.14432 & 165.98720 & \\
\hline
\end{tabular}


tert-butyl 2-((phenyl(o-tolyl)methyl)amino)acetate (2v)
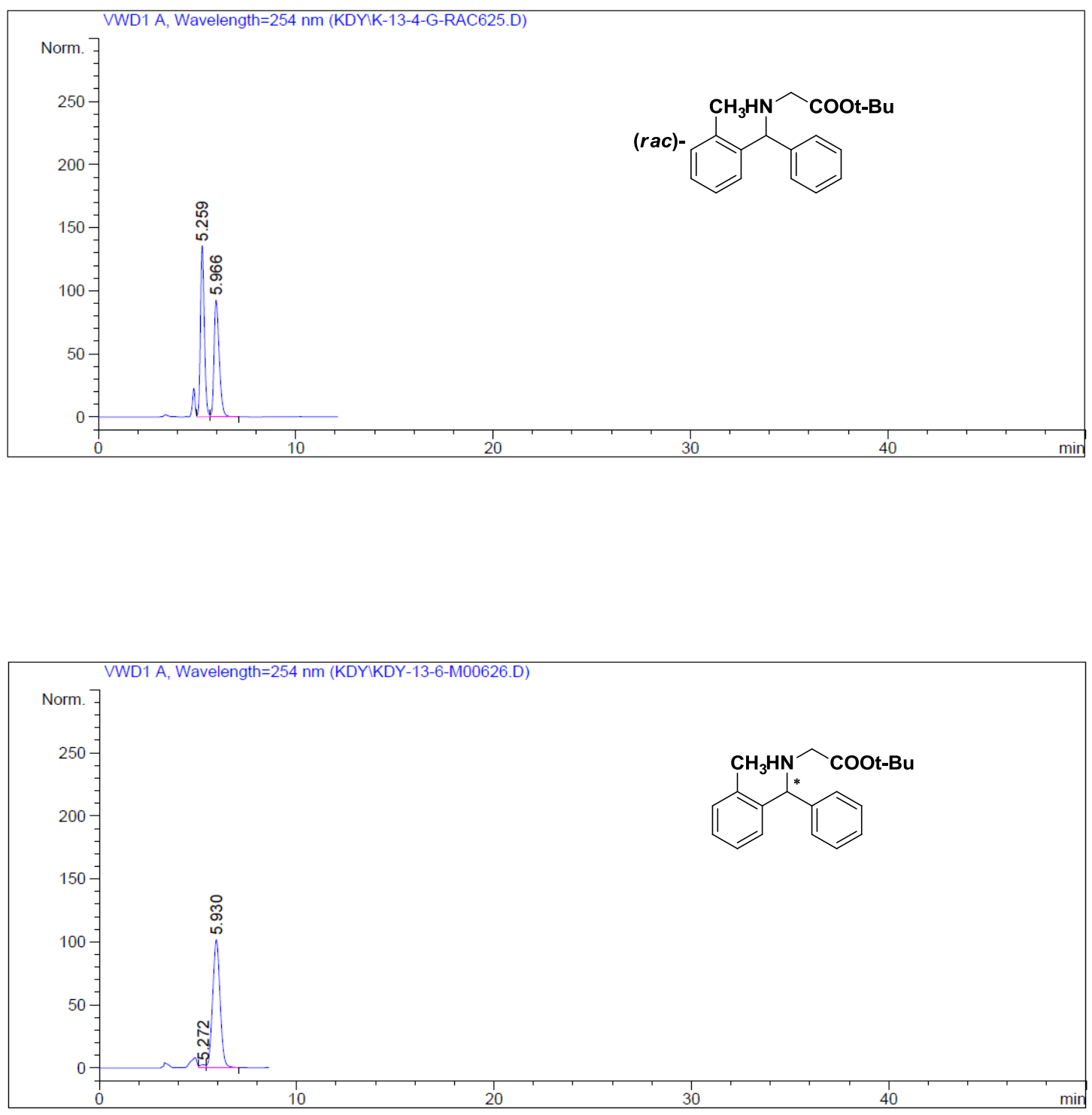

\begin{tabular}{|c|c|c|c|c|c|c|}
\hline $\begin{array}{c}\text { Peak } \\
\quad \#\end{array}$ & $\begin{array}{c}\text { RetTime } \\
\text { [min] }\end{array}$ & Type & $\begin{array}{c}\text { Width } \\
{[\mathrm{min}]}\end{array}$ & $\begin{array}{c}\text { Area } \\
{\left[\mathrm{mAU} \mathrm{U}^{*} \mathrm{~S}\right]}\end{array}$ & $\begin{array}{l}\text { Height } \\
{[\mathrm{mAU}]}\end{array}$ & $\begin{array}{c}\text { Area } \\
\frac{\circ}{\circ}\end{array}$ \\
\hline- & ----- & & ----- & | ---------- & -------- & ------ \\
\hline 1 & 5.272 & VV & 0.2750 & 42.56733 & 2.26759 & 1.5821 \\
\hline 2 & 5.930 & VB & 0.4036 & 2647.92725 & 101.42326 & 98.4179 \\
\hline ta & & & & 2690.49457 & .69085 & \\
\hline
\end{tabular}




\section{$N$-methyl-1-phenyl-1-(o-tolyl)methanamine(2w)}
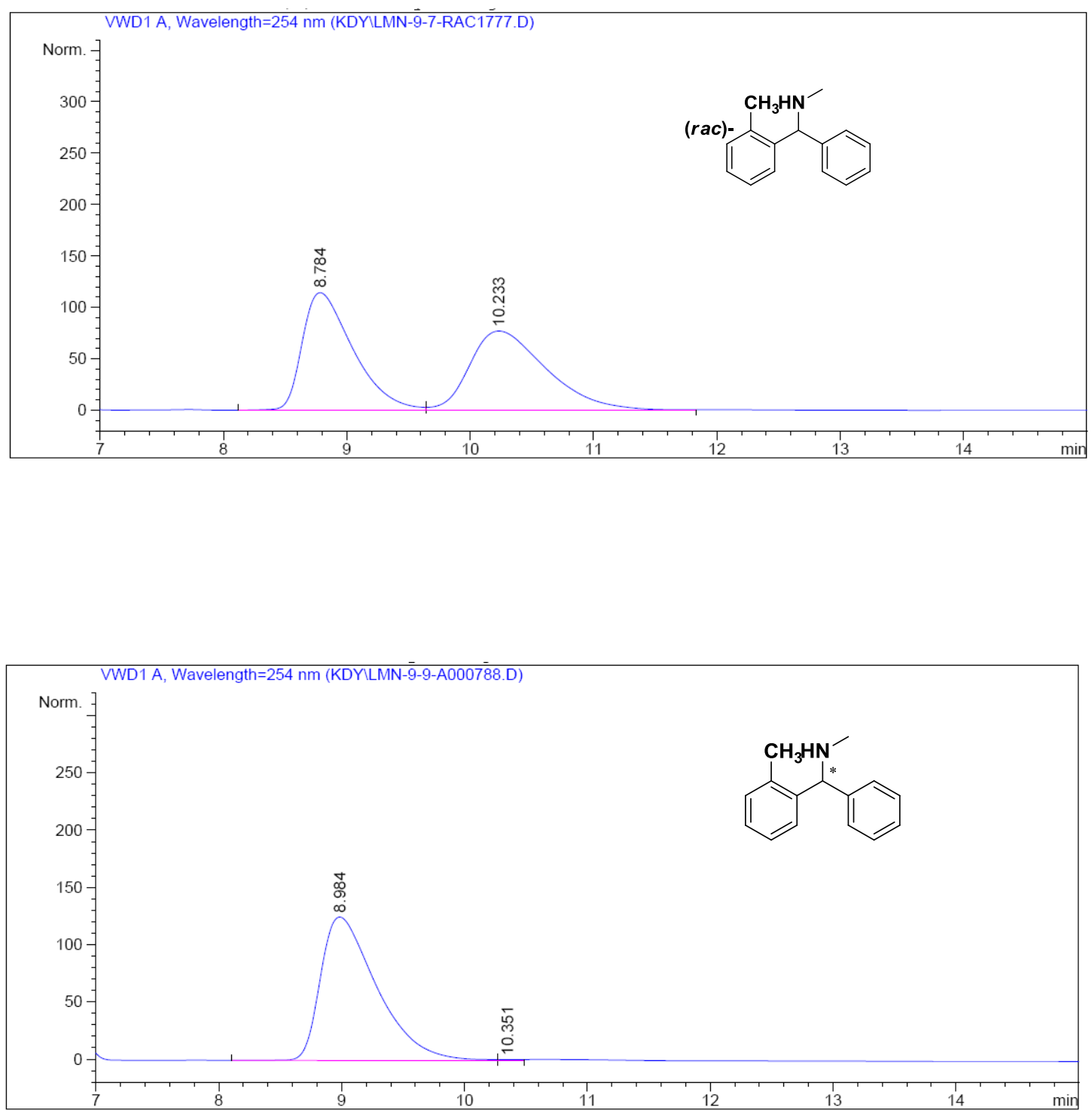

\begin{tabular}{|c|c|c|c|c|c|c|}
\hline $\begin{array}{c}\text { Peak } \\
\quad \#\end{array}$ & $\begin{array}{c}\text { RetTime } \\
\text { [min] }\end{array}$ & Type & $\begin{array}{c}\text { Width } \\
{[\mathrm{min}]}\end{array}$ & $\begin{array}{c}\text { Area } \\
{\left[\mathrm{mAU}^{*} \mathrm{~s}\right]}\end{array}$ & $\begin{array}{l}\text { Height } \\
{[\mathrm{mAU}]}\end{array}$ & $\begin{array}{c}\text { Area } \\
\frac{\circ}{6}\end{array}$ \\
\hline-- & $\begin{array}{c}------- \\
8\end{array}$ & & $-x-10$ & $\begin{array}{l}----1--1- \\
399367651\end{array}$ & ---------1 & $\begin{array}{l}----- \\
7096\end{array}$ \\
\hline $\begin{array}{l}1 \\
2\end{array}$ & $\begin{array}{r}8.984 \\
10.351\end{array}$ & $\begin{array}{l}\text { BV } \\
\text { VV }\end{array}$ & $\begin{array}{l}0.4809 \\
0.1742\end{array}$ & $\begin{array}{r}3993.67651 \\
11.63242\end{array}$ & $\begin{array}{r}125.27110 \\
9.09494 e-1\end{array}$ & $\begin{array}{r}99.7096 \\
0.2904\end{array}$ \\
\hline
\end{tabular}

Totals :

$4005.30893 \quad 126.18060$ 
$N$-(phenyl(o-tolyl)methyl)ethanamine(2x)
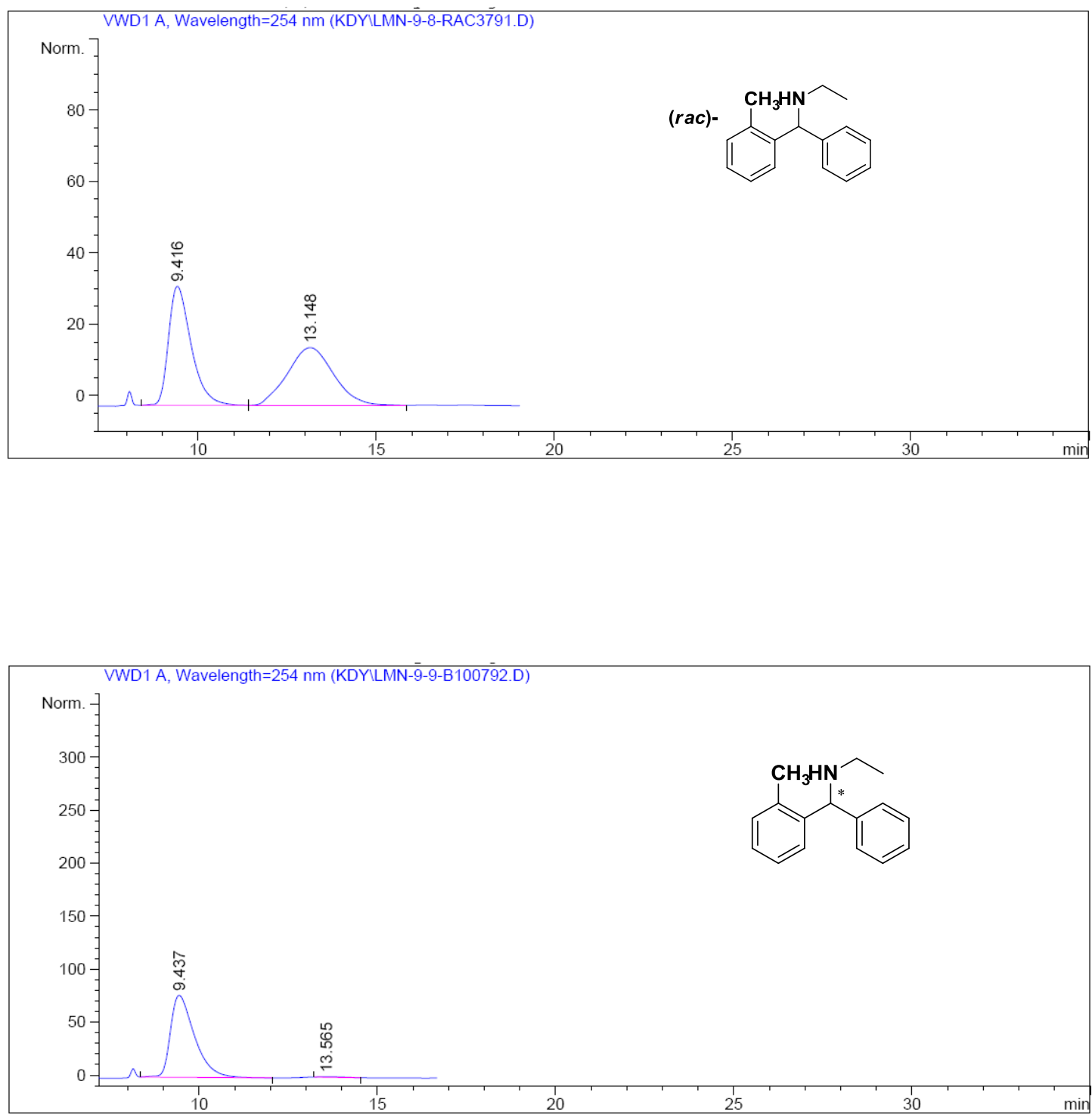

\begin{tabular}{|c|c|c|c|c|c|c|}
\hline $\begin{array}{c}\text { eak } \\
\#\end{array}$ & $\begin{array}{c}\text { RetTime } \\
\text { [min] }\end{array}$ & Type & $\begin{array}{l}\text { Width } \\
\text { [min] }\end{array}$ & $\begin{array}{c}\text { Area } \\
{\left[\mathrm{mAU}^{*} \mathrm{~S}\right]}\end{array}$ & $\begin{array}{l}\text { Height } \\
{[\mathrm{mAU}]}\end{array}$ & $\begin{array}{c}\text { Area } \\
\quad \%\end{array}$ \\
\hline 1 & & & & 3617.46 & & 99 \\
\hline 2 & 13.5 & & 5877 & 19.97696 & $4.09113 e-1$ & 5492 \\
\hline
\end{tabular}

Totals :

$3637.44644 \quad 77.68428$ 
methyl 2-((phenyl(m-tolyl)methyl)amino)acetate (3a)
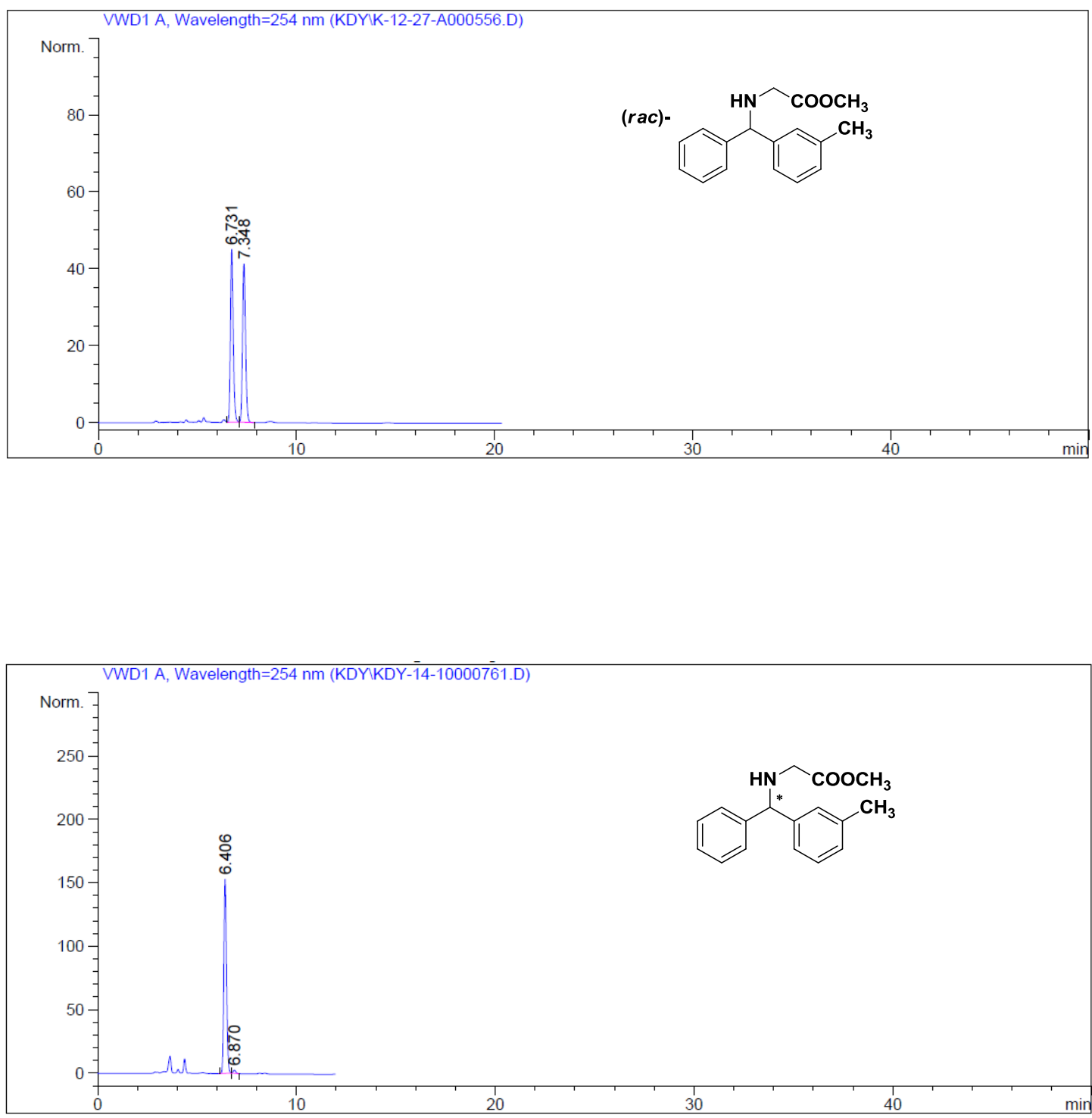

\begin{tabular}{|c|c|c|c|c|c|c|}
\hline $\begin{array}{c}\text { Peak } \\
\text { \# }\end{array}$ & $\begin{array}{c}\text { RetTime } \\
\text { [min] }\end{array}$ & Type & $\begin{array}{l}\text { Width } \\
\text { [min] }\end{array}$ & $\begin{array}{c}\text { Area } \\
{\left[\mathrm{mAU}^{*} \mathrm{~S}\right]}\end{array}$ & $\begin{array}{l}\text { Height } \\
\text { [mAU] }\end{array}$ & $\begin{array}{c}\text { Area } \\
\frac{\circ}{\circ}\end{array}$ \\
\hline & & & & 1437 & 52.9 & \\
\hline 2 & 6.870 & & 0.1437 & 22.39592 & 2.44923 & 5338 \\
\hline
\end{tabular}

Totals :

$1460.18547 \quad 155.37970$ 
methyl 2-((phenyl(p-tolyl)methyl)amino)acetate (3b)
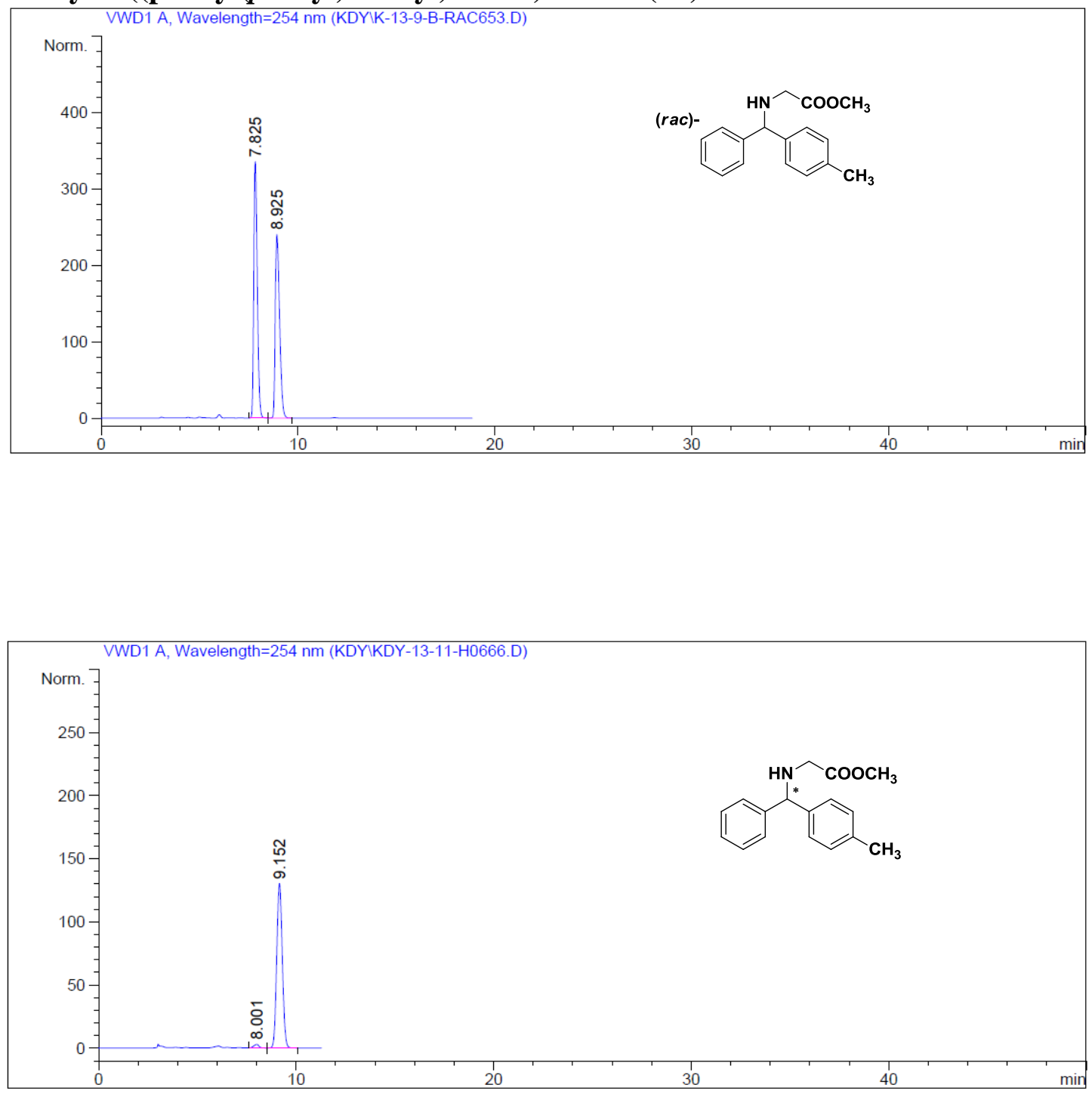

\begin{tabular}{|c|c|c|c|c|c|c|}
\hline $\begin{array}{l}\text { Peak } \\
\quad \#\end{array}$ & $\begin{array}{c}\text { RetTime } \\
\text { [min] }\end{array}$ & Type & $\begin{array}{l}\text { Width } \\
{[\mathrm{min}]}\end{array}$ & $\begin{array}{c}\text { Area } \\
{\left[\mathrm{mAU}^{*} \mathrm{~S}\right]}\end{array}$ & $\begin{array}{l}\text { Height } \\
\text { [mAU] }\end{array}$ & $\begin{array}{c}\text { Area } \\
\quad \frac{\circ}{0}\end{array}$ \\
\hline & & & ----- & |--------- & & \\
\hline 1 & 8.001 & BB & 0.2773 & 48.32494 & 2.77283 & 1.8605 \\
\hline 2 & 9.152 & BB & 0.3086 & 2549.03394 & 130.10681 & 98.1395 \\
\hline tal & : & & & 2597.35887 & 132.87965 & \\
\hline
\end{tabular}


methyl 2-(((3-fluorophenyl)(phenyl)methyl)amino)acetate (3c)
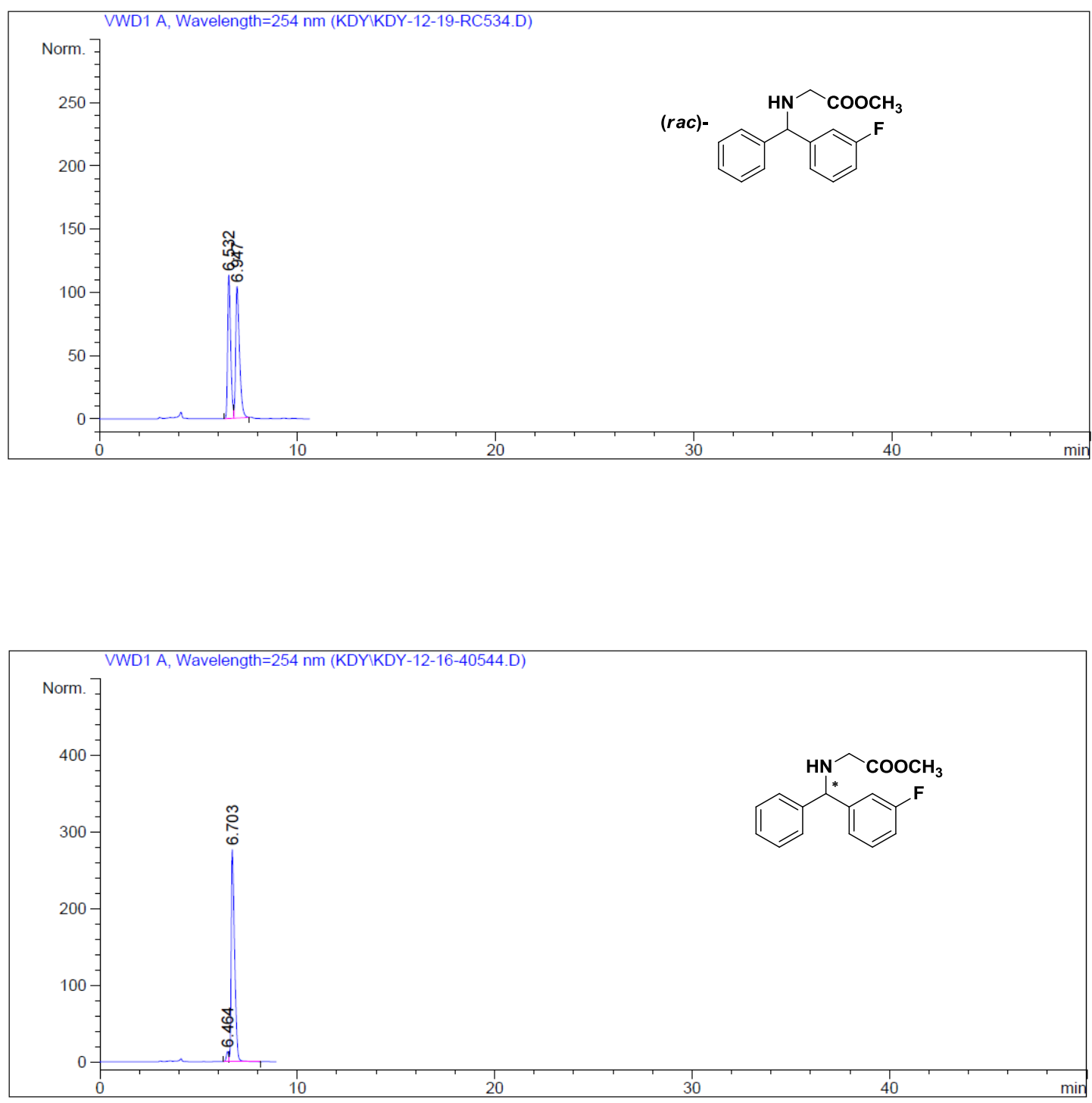

\begin{tabular}{|c|c|c|c|c|c|c|}
\hline $\begin{array}{c}\text { Peak } \\
\quad \#\end{array}$ & $\begin{array}{c}\text { RetTime } \\
\text { [min] }\end{array}$ & Type & $\begin{array}{c}\text { Width } \\
{[\mathrm{min}]}\end{array}$ & $\begin{array}{c}\text { Area } \\
{\left[\mathrm{mAU}^{*} \mathrm{~S}\right]}\end{array}$ & $\begin{array}{l}\text { Height } \\
\text { [mAU] }\end{array}$ & $\begin{array}{c}\text { Area } \\
\text { 응 }\end{array}$ \\
\hline 1 & 6.464 & $\mathrm{BV}$ & 0.1271 & 107.48776 & 13.19317 & 3.1508 \\
\hline 2 & 6.703 & $\mathrm{VB}$ & 0.1774 & 3303.94434 & 276.47818 & 96.8492 \\
\hline Tota & $\mathrm{s}:$ & & & 3411.43210 & 289.67135 & \\
\hline
\end{tabular}


methyl 2-(((4-fluorophenyl)(phenyl)methyl)amino)acetate (3d)
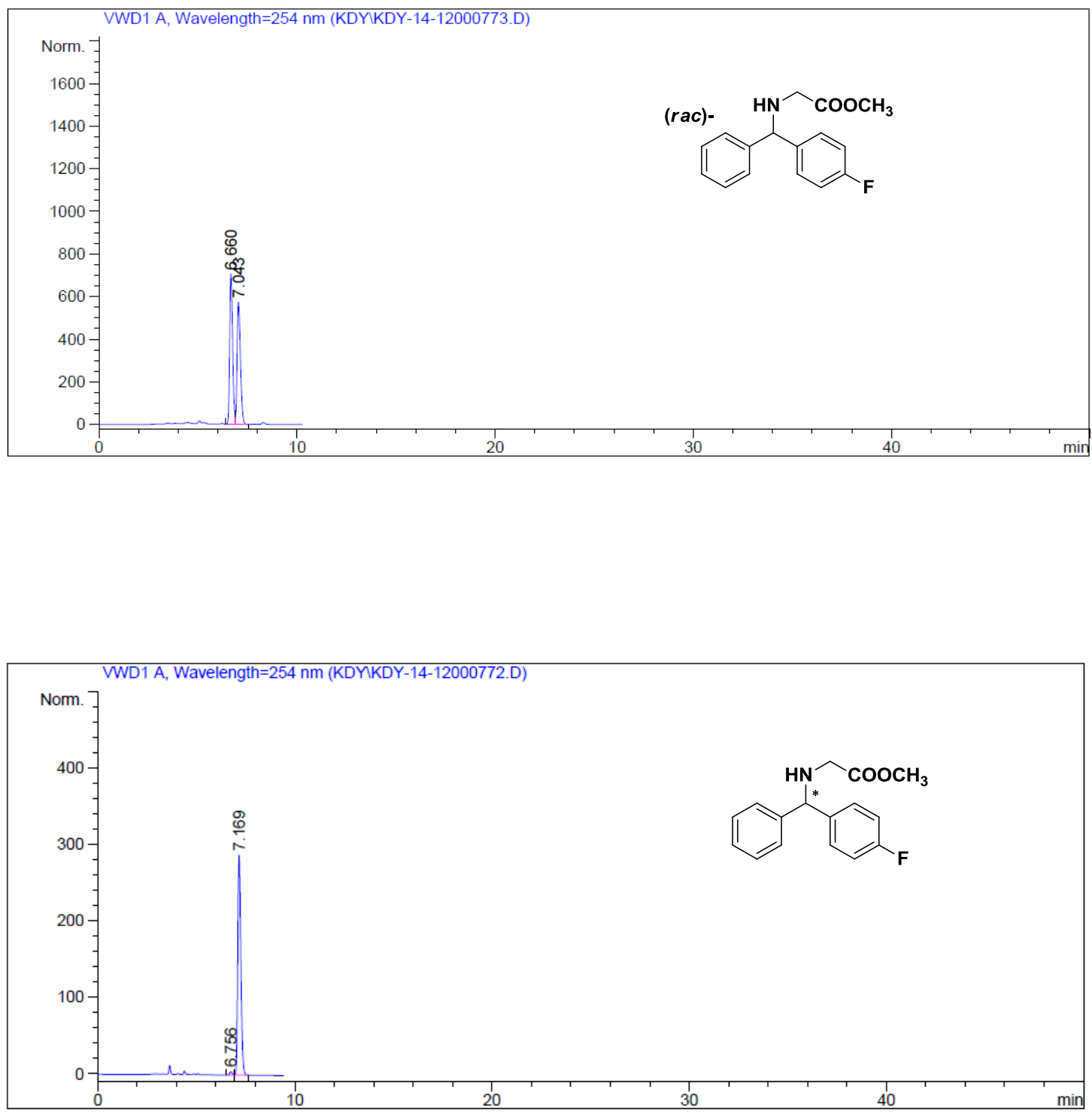

\begin{tabular}{|c|c|c|c|c|c|c|}
\hline $\begin{array}{c}\text { Peak } \\
\quad \#\end{array}$ & $\begin{array}{l}\text { RetTime } \\
\text { [min] }\end{array}$ & Type & $\begin{array}{l}\text { Width } \\
\text { [min] }\end{array}$ & $\begin{array}{c}\text { Area } \\
{\left[\mathrm{mAU}^{\star} \mathrm{s}\right]}\end{array}$ & $\begin{array}{l}\text { Height } \\
\text { [mAU] }\end{array}$ & $\begin{array}{c}\text { Area } \\
\text { \% }\end{array}$ \\
\hline--1 & |------- & & ------- & ---------- & ---------- & $-------\mid$ \\
\hline 1 & 6.756 & $\mathrm{BB}$ & 0.1429 & 37.27180 & 4.06789 & 1.1806 \\
\hline 2 & 7.169 & $\mathrm{BB}$ & 0.1688 & 3119.69312 & 287.09259 & 98.8194 \\
\hline Total & : & & & 3156.96492 & 291.16048 & \\
\hline
\end{tabular}


methyl 2-(((4-chlorophenyl)(phenyl)methyl)amino)acetate (3e)
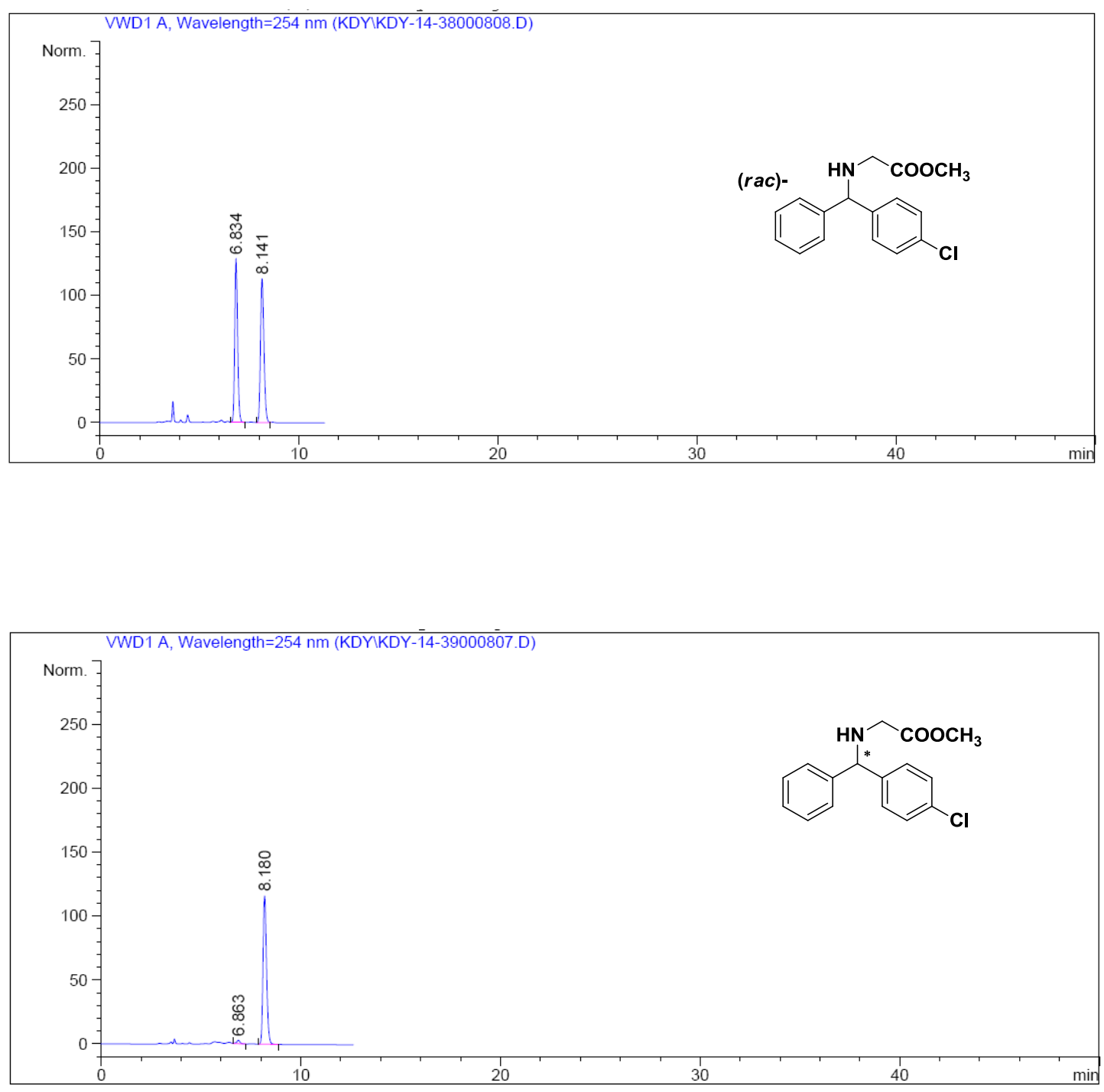

Signal 1: VWD1 A, Wavelength=254 nm

\begin{tabular}{|c|c|c|c|c|c|c|}
\hline $\begin{array}{c}\text { Peak } \\
\#\end{array}$ & $\begin{array}{c}\text { RetTime } \\
\text { [min] }\end{array}$ & Type & $\begin{array}{c}\text { Width } \\
\text { [min] }\end{array}$ & $\begin{array}{c}\text { Area } \\
{\left[\mathrm{mAU}^{*} \mathrm{~S}\right]}\end{array}$ & $\begin{array}{l}\text { Height } \\
{[\mathrm{mAU}]}\end{array}$ & $\begin{array}{c}\text { Area } \\
\frac{\circ}{\circ}\end{array}$ \\
\hline 1 & 6.863 & VB & 0.1757 & 32.45758 & 2.71110 & 2.2378 \\
\hline 2 & 8.180 & $\mathrm{BB}$ & 0.1885 & 1417.94116 & 116.04850 & 97.7622 \\
\hline Total & : & & & 1450.39875 & 118.75960 & \\
\hline
\end{tabular}


ethyl 2-(((4-chlorophenyl)(phenyl)methyl)(2-methoxy-2-oxoethyl)amino)acetate (4)
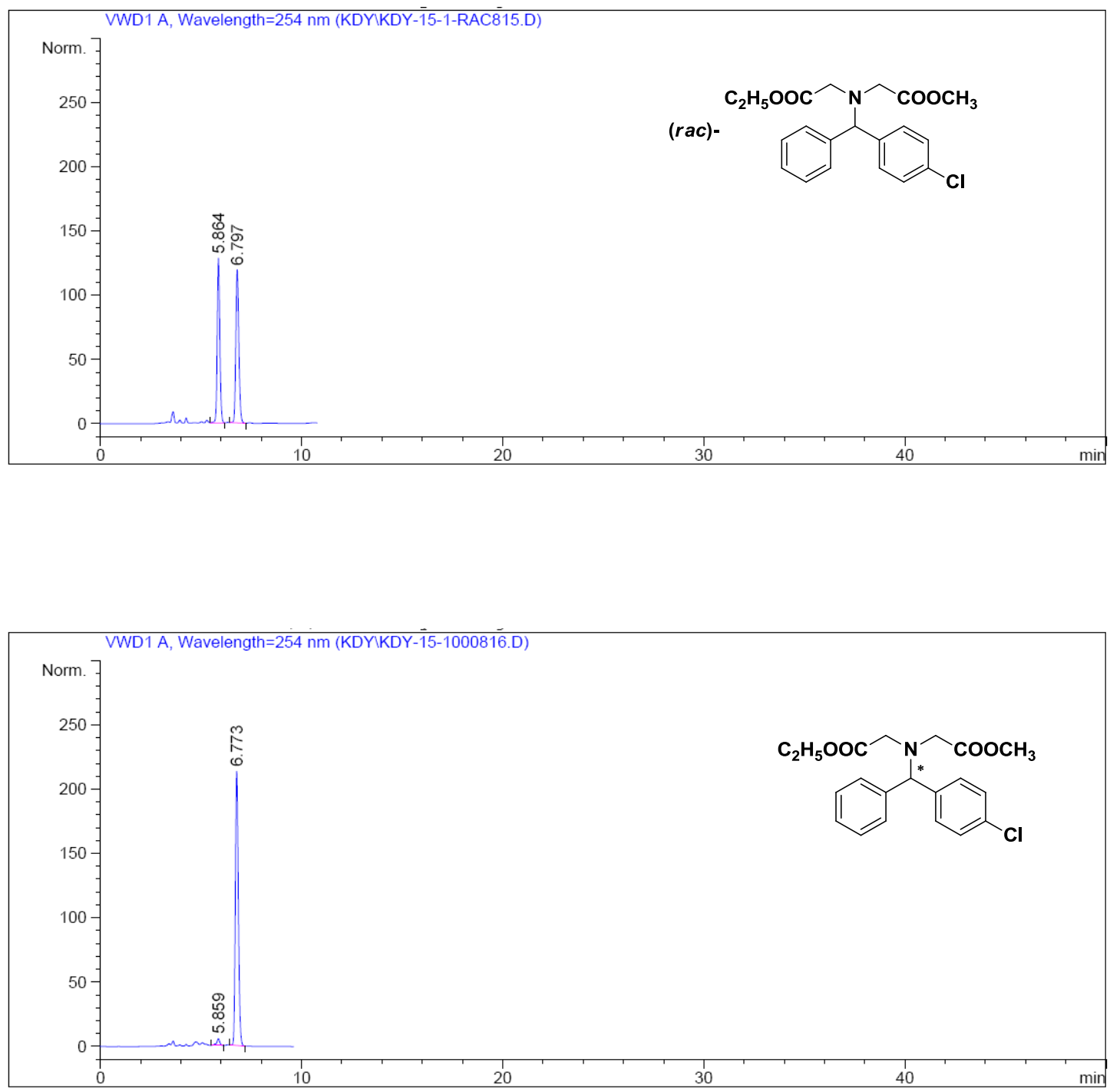

Signal 1: VWD1 A, Wavelength=254 nm

\begin{tabular}{|c|c|c|c|c|c|c|}
\hline $\begin{array}{c}\text { Peak } \\
\quad \#\end{array}$ & $\begin{array}{c}\text { RetTime } \\
\text { [min] }\end{array}$ & Type & $\begin{array}{l}\text { Width } \\
\text { [min] }\end{array}$ & $\begin{array}{c}\text { Area } \\
{\left[\mathrm{mAU}^{*} \mathrm{~S}\right]}\end{array}$ & $\begin{array}{l}\text { Height } \\
{[\mathrm{mAU}]}\end{array}$ & $\begin{array}{c}\text { Area } \\
\quad \frac{\circ}{\partial}\end{array}$ \\
\hline 1 & 5.859 & $\mathrm{BB}$ & 0.1576 & 56.47129 & 5.24604 & 2.4868 \\
\hline 2 & 6.773 & $\mathrm{BB}$ & 0.1602 & 2214.34326 & 213.16209 & 97.5132 \\
\hline $\mathrm{Eal}$ & . & & & 2270.81455 & 218.40813 & \\
\hline
\end{tabular}


phenyl(o-tolyl)methanamine (5a)
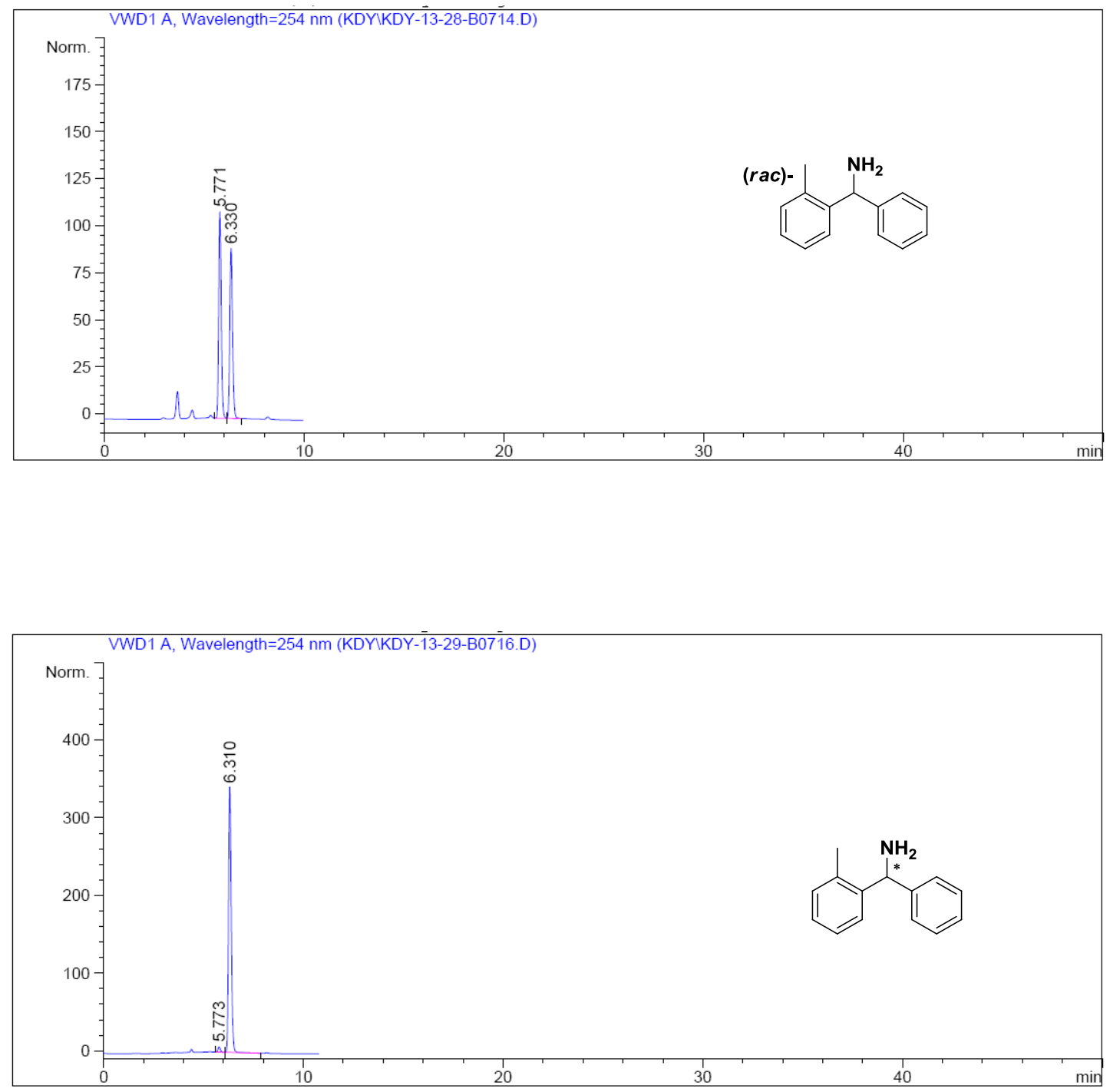

Signal 1: VWD1 A, Wavelength=254 nm

\begin{tabular}{|c|c|c|c|c|c|c|}
\hline $\begin{array}{c}\text { Peak } \\
\#\end{array}$ & $\begin{array}{c}\text { RetTime } \\
\text { [min] }\end{array}$ & Type & $\begin{array}{c}\text { Width } \\
\text { [min] }\end{array}$ & $\begin{array}{c}\text { Area } \\
{\left[\mathrm{mAU}^{*} \mathrm{~s}\right]}\end{array}$ & $\begin{array}{l}\text { Height } \\
{[\mathrm{mAU}]}\end{array}$ & $\begin{array}{c}\text { Area } \\
\frac{\circ}{b}\end{array}$ \\
\hline-- & ------ & -7 & ------- & |---------- & ---------- & -------- \\
\hline 1 & 5.773 & BB & 0.1273 & 56.53692 & 6.84856 & 1.7283 \\
\hline 2 & 6.310 & $\mathrm{BB}$ & 0.1458 & 3214.78760 & 341.62454 & 98.2717 \\
\hline al & & & & 3271.32452 & 348.47310 & \\
\hline
\end{tabular}


(4-chlorophenyl)(phenyl)methanamine (5b)
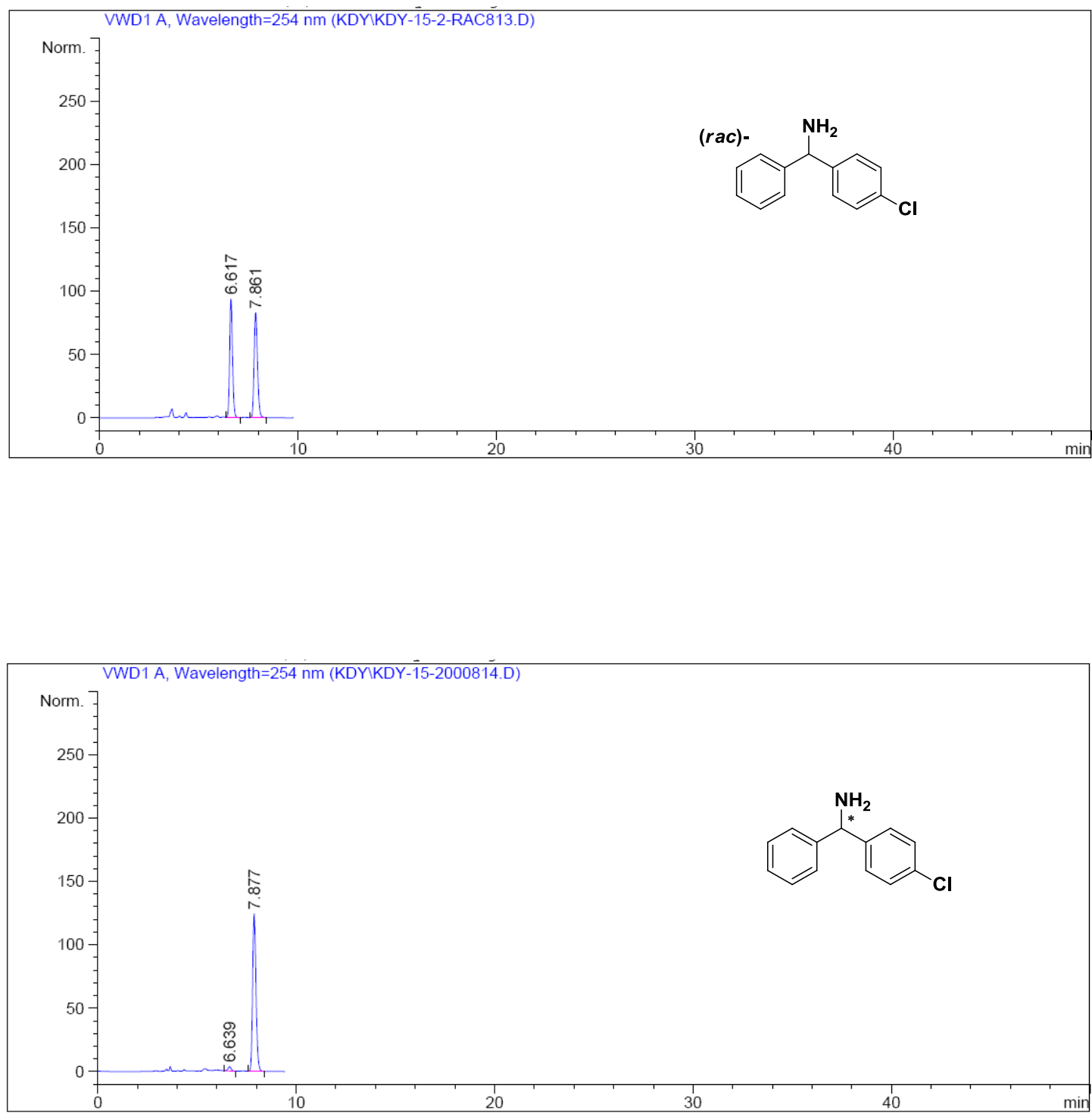

\begin{tabular}{|c|c|c|c|c|c|c|}
\hline $\begin{array}{c}\text { Peak } \\
\quad \#\end{array}$ & $\begin{array}{c}\text { RetTime } \\
\text { [min] }\end{array}$ & Type & $\begin{array}{c}\text { Width } \\
\text { [min] }\end{array}$ & $\begin{array}{c}\text { Area } \\
{\left[\mathrm{mAU}^{*} \mathrm{~s}\right]}\end{array}$ & $\begin{array}{l}\text { Height } \\
{[\mathrm{mAU}]}\end{array}$ & $\begin{array}{c}\text { Area } \\
\frac{\circ}{0}\end{array}$ \\
\hline & & & & | ----------- & --------- & ------- \\
\hline 1 & 6.639 & $\mathrm{BB}$ & 0.1631 & 37.65041 & 3.45476 & 2.4909 \\
\hline 2 & 7.877 & $\mathrm{BB}$ & 0.1834 & 1473.88843 & 124.21442 & 97.5091 \\
\hline Total & : & & & 1511.53883 & 127.66918 & \\
\hline
\end{tabular}

